\title{
Review Article \\ On Bondage Numbers of Graphs: A Survey with Some Comments
}

\begin{abstract}
Jun-Ming Xu
School of Mathematical Sciences, University of Science and Technology of China, Wentsun Wu Key Laboratory of CAS, Hefei, Anhui 230026, China
\end{abstract}

Correspondence should be addressed to Jun-Ming Xu; xujm@ustc.edu.cn

Received 15 December 2012; Revised 20 February 2013; Accepted 11 March 2013

Academic Editor: Chính T. Hoang

Copyright (C) 2013 Jun-Ming Xu. This is an open access article distributed under the Creative Commons Attribution License, which permits unrestricted use, distribution, and reproduction in any medium, provided the original work is properly cited.

The domination number of a graph $G$ is the smallest number of vertices which dominate all remaining vertices by edges of $G$. The bondage number of a nonempty graph $G$ is the smallest number of edges whose removal from $G$ results in a graph with domination number greater than the domination number of $G$. The concept of the bondage number was formally introduced by Fink et al. in 1990. Since then, this topic has received considerable research attention and made some progress, variations, and generalizations. This paper gives a survey on the bondage number, including known results, conjectures, problems, and some comments, also selectively summarizes other types of bondage numbers.

\section{Introduction}

For terminology and notation on graph theory not given here, the reader is referred to $\mathrm{Xu}[1]$. Let $G=(V, E)$ be a finite, undirected, and simple graph. We call $|V|$ and $|E|$ the order and size of $G$ and denote them by $v=v(G)$ and $\varepsilon=\varepsilon(G)$, respectively, unless otherwise specified. Through this paper, the notations $P_{n}, C_{n}$, and $K_{n}$ always denote a path, a cycle, and a complete graph of order $n$, respectively, the notation $K_{n_{1}, n_{2}, \ldots, n_{t}}$ denotes a complete $t$-partite graph with $n_{1} \leqslant n_{2} \leqslant$ $\cdots \leqslant n_{t}$ and $n_{t}>1, K_{t}(m)=K_{n_{1}, n_{2}, \ldots, n_{t}}$ with $n_{1}=\cdots=n_{t}=$ $m$, and $K_{1, n-1}$ is a star. For two vertices $x$ and $y$ in a connected graph $G$, we use $d_{G}(x, y)$ to denote the distance between $x$ and $y$ in $G$.

For a vertex $x$ in $G$, let $N_{G}(x)$ be the open set of neighbors of $x$ and $N_{G}[x]=N[x]=N_{G}(x) \cup\{x\}$ the closed set of neighbors of $x$. For a subset $X \subset V(G), N_{G}(X)=$ $\left(\cup_{x \in X} N_{G}(x)\right) \cap \bar{X}$, and $N_{G}[X]=N_{G}(X) \cup X$, where $\bar{X}=$ $V(G) \backslash X$. Let $E_{x}$ be the set of edges incident with $x$ in $G$; that is, $E_{x}=\left\{x y \in E(G): y \in N_{G}(x)\right\}$. We denote the degree of $x$ by $d_{G}(x)=\left|E_{x}\right|$. The maximum and the minimum degrees of $G$ are denoted by $\Delta(G)$ and $\delta(G)$, respectively. A vertex of degree zero is called an isolated vertex. An edge incident with a vertex of degree one is called a pendant edge.
The bondage number is an important parameter of graphs which is based upon the well-known domination number.

A subset $S \subseteq V(G)$ is called a dominating set of $G$ if $N_{G}[S]=V(G)$; that is, every vertex $x$ in $\bar{S}$ has at least one neighbor in $S$. The domination number of $G$, denoted by $\gamma(G)$, is the minimum cardinality among all dominating sets; that is,

$$
\gamma(G)=\min \left\{|S|: S \subseteq V(G), N_{G}[S]=V(G)\right\} .
$$

A dominating set $S$ is called a $\gamma$-set of $G$ if $|S|=\gamma(G)$.

The domination is such an important and classic conception that it has become one of the most widely studied topics in graph theory and also is frequently used to study property of networks. The domination, with many variations and generalizations, is now well studied in graph and networks theory. The early vast literature on domination includes the bibliography compiled by Hedetniemi and Laskar [2] and a thorough study of domination appears in the books by Haynes et al. [3, 4]. However, the problem determining the domination number for general graphs was early proved to be NP-complete (see GT2 in Appendix in Garey and Johnson [5], 1979).

Among various problems related with the domination number, some focus on graph alterations and their effects 
on the domination number. Here, we are concerned with a particular graph alternation, the removal of edges from a graph.

Graphs with domination numbers changed upon the removal of an edge were first investigated by Walikar and Acharya [6] in 1979. A graph is called edge-dominationcritical graph if $\gamma(G-e)>\gamma(G)$ for every edge $e$ in $G$. The edge-domination-critical graph was characterized by Bauer et al. [7] in 1983; that is, a graph is edge-domination-critical if and only if it is the union of stars.

However, for lots of graphs, the domination number is out of the range of one-edge removal. It is immediate that $\gamma(H) \geqslant$ $\gamma(G)$ for any spanning subgraph $H$ of $G$. Every graph $G$ has a spanning forest $T$ with $\gamma(G)=\gamma(T)$, and so, in general, a graph has a nonempty subset $F \subseteq E(G)$ for which $\gamma(G-F)=$ $\gamma(G)$

Then it is natural for the alternation to be generalized to the removal of several edges, which is just enough to enlarge the domination number. That is the idea of the bondage number.

A measure of the efficiency of a domination in graphs was first given by Bauer et al. [7] in 1983, who called this measure as domination line-stability, defined as the minimum number of lines (i.e., edges) which when removed from $G$ increases $\gamma$.

In 1990, Fink et al. [8] formally introduced the bondage number as a parameter for measuring the vulnerability of the interconnection network under link failure. The minimum dominating set of sites plays an important role in the network for it dominates the whole network with the minimum cost. So we must consider whether its function remains good when the network is attacked. Suppose that someone such as a saboteur does not know which sites in the network take part in the dominating role, but does know that the set of these special sites corresponds to a minimum dominating set in the related graph. Then how many links does he has to attack so that the cost cannot remains the same in order to dominate the whole network? That minimum number of links is just the bondage number.

The bondage number $b(G)$ of a nonempty undirected graph $G$ is the minimum number of edges whose removal from $G$ results in a graph with larger domination number. The precise definition of the bondage number is as follows:

$$
b(G)=\min \{|B|: B \subseteq E(G), \gamma(G-B)>\gamma(G)\} .
$$

Since the domination number of every spanning subgraph of a nonempty graph $G$ is at least as great as $\gamma(G)$, the bondage number of a nonempty graph is well defined.

We call such an edge-set $B$ with $\gamma(G-B)>\gamma(G)$ the bondage set and the minimum one the minimum bondage set. In fact, if $B$ is a minimum bondage set, then $\gamma(G-B)=\gamma(G)+1$ because the removal of one single edge cannot increase the domination number by more than one. If $b(G)$ does not exist, for example, empty graphs, we define $b(G)=\infty$.

It is quite difficult to compute the exact value of the bondage number for general graphs since it strongly depends on the domination number of the graphs. Much work focused on the bounds on the bondage number as well as the restraints on some particular classes of graphs or networks. The purpose of this paper is to give a survey of results and research methods related to these topics for graphs and digraphs. For some results and research methods, we will make some comments to develop our further study.

The rest of the paper is organized as follows. Section 2 gives some preliminary results and complexity. Sections 3 and 4 survey some upper bounds and lower bounds, respectively. The results for some special classes of graphs and planar graphs are stated in Sections 5 and 6, respectively. In Section 7, we introduce some results on crossing number restraints. In Sections 8 and 9, we are concerned about other and generalized types of bondage numbers, respectively. In Section 10, we introduce some results for digraphs. In the last section, we introduce some results for vertex-transitive graphs by applying efficient dominating sets.

\section{Simplicity and Complexity}

As we have known from Introduction, the bondage number is an important parameter for measuring the stability or the vulnerability of a domination in a graph or a network. Our aim is to compute the bondage number for any given graphs or networks. One has determined the exact value of the bondage number for some graphs with simple structure. For arbitrarily given graph, however, it has been proved that determining its bondage number is NP-hard.

2.1. Exact Values for Ordinary Graphs. We begin our investigation of the bondage number by computing its value for several well-known classes of graphs with simple structure. In 1990, Fink et al. [8] proposed the concept of the bondage number and completely determined the exact values of bondage numbers of some ordinary graphs, such as complete graphs, paths, cycles, and complete multipartite graphs.

Theorem 1 (Fink et al. [8], 1990). The exact values of bondage numbers of the following class of graphs are completely determined:

$$
\begin{gathered}
b\left(K_{n}\right)=\left\lceil\frac{n}{2}\right\rceil ; \\
b\left(P_{n}\right)= \begin{cases}2 & \text { if } n \equiv 1(\bmod 3), \\
1 & \text { otherwise, }\end{cases} \\
b\left(K_{n_{1}, n_{2}, \ldots, n_{t}}\right)= \begin{cases}3 & \text { if } n \equiv 1(\bmod 3), \\
2 & \text { otherwise, }\end{cases} \\
= \begin{cases}\left\lceil\frac{j}{2}\right\rceil & \text { if } n_{j}=1 \text { and } n_{j+1} \geqslant 2, \text { for some } j, \\
2 t-1 \quad \text { if } n_{1}=n_{2}=\cdots=n_{t}=2, \\
\sum_{i=1}^{t-1} n_{i} \quad \text { otherwise. }\end{cases}
\end{gathered}
$$

The complete graph $K_{n}$ is the unique $(n-1)$-regular graph of order $n \geqslant 2, b\left(K_{n}\right)=\lceil n / 2\rceil$ by Theorem 1 . The $t$-partite 
graph $K_{t}(2)$ with $t=n / 2$ is an $(n-2)$-regular graph $G$ of order $n \geqslant 2$, and not unique, $b(G)=n-1$ by Theorem 1 for an even integer $n \geqslant 4$. For an $(n-3)$-regular graph $\mathrm{G}$ of order $n \geqslant 4, \mathrm{Hu}$ and $\mathrm{Xu}$ [9] obtained the following result.

Theorem 2 (Hu and $\mathrm{Xu}[9]) . b(G)=n-3$ for any $(n-3)$ regular graph $G$ of order $n \geqslant 4$.

Up to the present, no results have been known for $k$ regular graphs with $3 \leqslant k \leqslant n-4$. For general graphs, there are the following two results.

Theorem 3 (Teschner [10], 1997). If $G$ is a nonempty graph with a unique minimum dominating set, then $b(G)=1$.

Theorem 4 (Bauer et al. [7], 1983). If any vertex of a graph $G$ is adjacent with two or more vertices of degree one, then $b(G)=1$.

Bauer et al. [7] observed that the star is the unique graph with the property that the bondage number is 1 and the deletion of any edge results in the domination number increasing. Motivated by this fact, Hartnell and Rall [11] proposed the concept of uniformly bonded graphs. A graph is called to be uniformly bonded if it has bondage number $b$ and the deletion of any $b$ edges results in a graph with increased domination number. Unfortunately, there are a few uniformly bonded graphs.

Theorem 5 (Hartnell and Rall [11], 1999). The only uniformly bonded graphs with bondage number 2 are $C_{3}$ and $P_{4}$. The unique uniformly bonded graph with bondage number 3 is $C_{4}$. There are no such graphs for bondage number greater than 3 .

As we mentioned, to compute the exact value of bondage number for a graph strongly depends upon its domination number. In this sense, studying the bondage number can greatly inspire one's research concerned with dominations. However, determining the exact value of domination number for a given graph is quite difficult. In fact, even if the exact value of the domination number for some graph is determined, it is still very difficulty to compute the value of the bondage number for that graph. For example, for the hypercube $Q_{n}$, we have $\gamma\left(Q_{n}\right)=2^{n-1}$, but we have not yet determined $b\left(Q_{n}\right)$ for any $n \geqslant 2$.

Perhaps Theorems 3 and 4 provide an approach to compute the exact value of bondage number for some graphs by establishing some sufficient conditions for $b(G)=b$. In fact, we will see later that Theorem 3 plays an important role in determining the exact values of the bondage numbers for some graphs. Thus, to study the bondage number, it is important to present various characterizations of graphs with a unique minimum dominating set.

2.2. Characterizations of Trees. For trees, Bauer et al. [7] in 1983 from the point of view of the domination line-stability, independently and Fink et al. [8] in 1990 from the point of view of the domination edge-vulnerability obtained the following result.

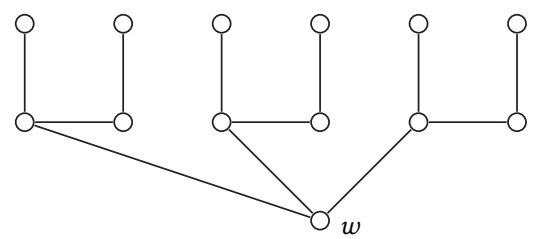

Figure 1: Tree $F_{t}$.

Theorem 6. For any nontrivial tree $T, b(T) \leqslant 2$.

By Theorem 6, it is natural to classify all trees according to their bondage numbers. Fink et al. [8] proved that a forbidden subgraph characterization to classify trees with different bondage numbers is impossible, since they proved that if $F$ is a forest, then $F$ is an induced subgraph of a tree $T$ with $b(T)=1$ and a tree $T^{\prime}$ with $b\left(T^{\prime}\right)=2$. However, they pointed out that the complexity of calculating the bondage number of a tree of order $n$ is at most $O\left(n^{2}\right)$ by methodically removing each pair of edges.

Even so, some characterizations, whether a tree has bondage number 1 or 2 , have been found by several authors; see, for example, $[10,12,13]$.

First we describe the method due to Hartnell and Rall [12], by which all trees with bondage number 2 can be constructed inductively. An important tree $F_{t}$ in the construction is shown in Figure 1. To characterize this construction, we need some terminologies:

(1) attach a path $P_{n}$ to a vertex $x$ of a tree, which means to link $x$ and one end-vertex of the $P_{n}$ by an edge.

(2) attach $F_{t}$ to a vertex $x$, which means to link $x$ and a vertex $y$ of $F_{t}$ by an edge.

The following are four operations on a tree $T$ :

Type 1: attach a $P_{2}$ to $x \in V(T)$, where $\gamma(T-x)=\gamma(T)$ and $x$ belongs to at least one $\gamma$-set of $T$ (such a vertex exists, say, one end-vertex of $P_{5}$ ).

Type 2: attach a $P_{3}$ to $x \in V(T)$, where $\gamma(T-x)<\gamma(T)$.

Type 3: attach $F_{1}$ to $x \in V(T)$, where $x$ belongs to at least one $\gamma$-set of $T$.

Type 4: attach $F_{t}, t \geqslant 2$, to $x \in V(T)$, where $x$ can be any vertex of $T$.

Let $\mathscr{C}=\left\{T: T\right.$ is a tree, and $T=K_{1}, T=P_{4}$, and $T=F_{t}$ for some $t \geqslant 2$, or $T$ can be obtained from $P_{4}$ or $F_{t}(t \geqslant 2)$ by a finite sequence of operations of Types $1,2,3,4\}$.

Theorem 7 (Hartnell and Rall [12], 1992). A tree has bondage number 2 if and only if it belongs to $\mathscr{C}$.

Looking at different minimum dominating sets of a tree, Teschner [10] presented a totally different characterization of the set of trees having bondage number 1 . They defined a vertex to be universal if it belongs to each minimum dominating set, and to be idle if it does not belong to any minimum dominating set. 
Theorem 8 (Teschner [10], 1997). A tree $T$ has bondage number 1 if and only if $T$ has a universal vertex or an edge xy satisfying

\section{(1) $x$ and $y$ are neither universal nor idle; and}

(2) all neighbors of $x$ and $y$ (except for $x$ and $y$ ) are idle.

For a positive integer $k$, a subset $I \subseteq V(G)$ is called a $k$ independent set (also called a $k$-packing) if $d_{G}(x, y)>k$ for any two distinct vertices $x$ and $y$ in $I$. When $k=1,1$-set is the normal independent set. The maximum cardinality among all $k$-independent sets is called the $k$-independence number (or $k$-packing number) of $G$, denoted by $\alpha_{k}(G)$. A $k$-independent set $I$ is called an $\alpha_{k}$-set if $|I|=\alpha_{k}(G)$. A graph $G$ is said to be $\alpha_{k}$-stable if $\alpha_{k}(G)=\alpha_{k}(G-e)$ for every edge $e$ of $G$. There are two important results on $k$-independent sets.

Proposition 9 (Topp and Vestergaard [13], 2000). A tree T is $\alpha_{k}$-stable if and only if T has a unique $\alpha_{k}$-set.

Proposition 10 (Meir and Moon [14], 1975). $\alpha_{2}(G) \leqslant \gamma(G)$ for any connected graph $G$ with equality for any tree.

Hartnell et al. [15], independently and Topp and Vestergaard [13] also gave a constructive characterization of trees with bondage number 2 and, applying Proposition 10, presented another characterization of those trees.

Theorem 11 (Hartnell et al. [15], 1998; Top and Vestergaard $[13], 2000) . b(T)=2$ for a tree $T$ if and only if $T$ has a unique $\alpha_{2}$-set.

According to this characterization, Hartnell et al. [15] presented a linear algorithm for determining the bondage number of a tree.

In this subsection, we introduce three characterizations for trees with bondage number 1 or 2 . The characterization in Theorem 7 is constructive, constructing all trees with bondage number 2, a natural and straightforward method, by a series of graph-operations. The characterization in Theorem 8 is a little advisable, by describing the inherent property of trees with bondage number 1 . The characterization in Theorem 11 is wonderful, by using a strong graphtheoretic concept, $\alpha_{k}$-set. In fact, this characterization is a byproduct of some results related to $\alpha_{k}$-sets for trees. It is that this characterization closed the relation between two concepts, the bondage number and the $k$-independent set, and hence is of research value and important significance.

2.3. Complexity for General Graphs. As mentioned above, the bondage number of a tree can be determined by a linear time algorithm. According to this algorithm, we can determine within polynomial time the domination number of any tree by removing each edge and verifying whether the domination number is enlarged according to the known linear time algorithm for domination numbers of trees.

However, it is impossible to find a polynomial time algorithm for bondage numbers of general graphs. If such an algorithm $A$ exists, then the domination number of any nonempty undirected graph $G$ can be determined within polynomial time by repeatedly using $A$. Let $G_{0}=G$ and $G_{i+1}=G_{i}-B_{i}$, where $B_{i}$ is the minimum edge set of $G_{i}$ found by $A$ such that $\gamma\left(G_{i}-B_{i}\right)=\gamma\left(G_{i-1}\right)+1$ for each $i=0,1, \ldots$; we can always find the minimum $B_{i}$ whose removal from $G_{i}$ enlarges the domination number, until $G_{k}=G_{k-1}-B_{k-1}$ is empty for some $k \geqslant 1$, though $B_{k-1}$ is not empty. Then $\gamma(G)=\gamma\left(G_{k}\right)-k=v(G)-k$. As known to all, if NP $\neq P$, the minimum dominating set problem is NP-complete, and so polynomial time algorithms for the bondage number do not exist unless $\mathrm{NP}=P$.

In fact, $\mathrm{Hu}$ and $\mathrm{Xu}[16]$ have recently shown that the problem determining the bondage number of general graphs is NP-hard.

Problem 1. Consider the decision problem:

$$
\begin{aligned}
& \text { Bondage Problem } \\
& \text { Instance: a graph } G \text { and a positive integer } b(\leqslant \varepsilon(G)) . \\
& \text { Question: is } b(G) \leqslant b \text { ? }
\end{aligned}
$$

Theorem 12 (Hu and Xu [16], 2012). The bondage problem is NP-hard.

The basic way of the proof is to follow Garey and Johnson's techniques for proving NP-hardness [5] by describing a polynomial transformation from the known NP-complete problem: 3 -satisfiability problem.

Theorem 12 shows that we are unable to find a polynomial time algorithm to determine bondage numbers of general graphs unless $\mathrm{NP}=P$. At the same time, this result also shows that the following study on the bondage number is of important significance.

(i) Find approximation polynomial algorithms with performance ratio as small as possible.

(ii) Find the lower and upper bounds with difference as small as possible.

(iii) Determine exact values for some graphs, specially well-known networks.

Unfortunately, we cannot prove whether or not determining the bondage number is NP-problem since for any subset $B \subset E(G)$ it is not clear that there is a polynomial algorithm to verify $\gamma(G-B)>\gamma(G)$. Since the problem of determining the domination number is NP-complete, we conjecture that it is not in NP. This is a worthwhile task to be studied further.

Motivated by the linear time algorithm of Hartnell et al. to compute the bondage number of a tree, we can make an attempt to consider whether there is a polynomial time algorithm to compute the bondage number for some special classes of graphs such as planar graphs, Cayley graphs, or graphs with some restrictions of graph-theoretical parameters such as degree, diameter, connectivity, and domination number.

\section{Upper Bounds}

By Theorem 12, since we cannot find a polynomial time algorithm for determining the exact values of bondage numbers 
of general graphs, it is weightily significative to establish some sharp bounds on the bondage number of a graph. In this section, we survey several known upper bounds on the bondage number in terms of some other graph-theoretical parameters.

3.1. Most Basic Upper Bounds. We start this subsection with a simple observation.

Observation 1 (Teschner [10], 1997). Let $H$ be a spanning subgraph obtained from a graph $G$ by removing $k$ edges. Then $b(G) \leqslant b(H)+k$.

If we select a spanning subgraph $H$ such that $b(H)=1$, then Observation 1 yields some upper bounds on the bondage number of a graph $G$. For example, take $H=G-E_{x}+e$ and $k=d_{G}(x)-1$, where $e \in E_{x}$, the following result can be obtained.

Theorem 13 (Bauer et al. [7], 1983). If there exists at least one vertex $x$ in a graph $G$ such that $\gamma(G-x) \geqslant \gamma(G)$, then $b(G) \leqslant$ $d_{G}(x) \leqslant \Delta(G)$.

The following early result obtained can be derived from Observation 1 by taking $H=G-E_{x y}$ and $k=d(x)+d(y)-2$.

Theorem 14 (Bauer et al. [7], 1983; Fink et al. [8], 1990). $b(G) \leqslant d(x)+d(y)-1$ for any two adjacent vertices $x$ and $y$ in a graph $G$; that is,

$$
b(G) \leqslant \min _{x y \in E(G)}\left\{d_{G}(x)+d_{G}(y)-1\right\} .
$$

This theorem gives a natural corollary obtained by several authors.

Corollary 15 (Bauer et al. [7], 1983; Fink et al. [8], 1990). If G is a graph without isolated vertices, then $b(G) \leqslant \Delta(G)+\delta(G)-1$.

In 1999, Hartnell and Rall [11] extended Theorem 14 to the following more general case, which can be also derived from Observation 1 by taking $H=G-E_{x}-E_{y}+(x, z, y)$ if $d_{G}(x, y)=2$, where $(x, z, y)$ is a path of length 2 in $G$.

Theorem 16 (Hartnell and Rall [11], 1999). $b(G) \leqslant d(x)+$ $d(y)-1$ for any distinct two vertices $x$ and $y$ in a graph $G$ with $d_{G}(x, y) \leqslant 2$; that is,

$$
b(G) \leqslant \min _{d_{G}(x, y) \leqslant 2}\left\{d_{G}(x)+d_{G}(y)-1\right\} .
$$

Corollary 17 (Fink et al. [8], 1990). If a vertex of a graph $G$ is adjacent with two or more vertices of degree one, then $b(G)=1$.

We remark that the bounds stated in Corollary 15 and Theorem 16 are sharp. As indicated by Theorem 1, one class of graphs in which the bondage number achieves these bounds is the class of cycles whose orders are congruent to 1 modulo 3 .

On the other hand, Hartnell and Rall [17] sharpened the upper bound in Theorem 14 as follows, which can be also derived from Observation 1.

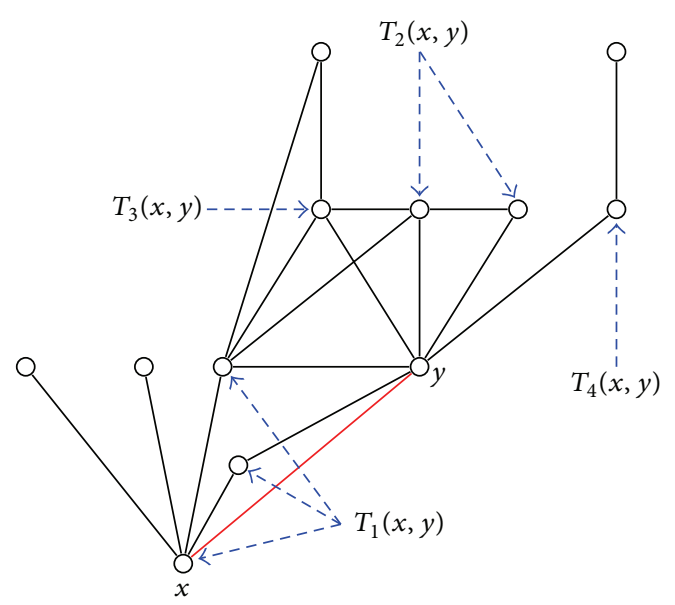

FIgURE 2: Illustration of $T_{i}(x, y)$ for $i=1,2,3,4$.

Theorem 18 (Hartnell and Rall [17], 1994). $b(G) \leqslant d_{G}(x)+$ $d_{G}(y)-1-\left|N_{G}(x) \cap N_{G}(y)\right|$ for any two adjacent vertices $x$ and $y$ in a graph $G$; that is,

$$
b(G) \leqslant \min _{x y \in E(G)}\left\{d_{G}(x)+d_{G}(y)-1-\left|N_{G}(x) \cap N_{G}(y)\right|\right\} .
$$

These results give simple but important upper bounds on the bondage number of a graph, and is also the foundation of almost all results on bondage numbers upper bounds obtained till now.

By careful consideration of the nature of the edges from the neighbors of $x$ and $y$, Wang [18] further refined the bound in Theorem 18. For any edge $x y \in E(G), N_{G}(y)$ contains the following four subsets:

(1) $T_{1}(x, y)=N_{G}[x] \cap N_{G}(y)$;

(2) $T_{2}(x, y)=\left\{w \in N_{G}(y): N_{G}(w) \subseteq N_{G}(y)-x\right\}$;

(3) $T_{3}(x, y)=\left\{w \in N_{G}(y): N_{G}(w) \subseteq N_{G}(z)-x\right.$ for some $\left.z \in N_{G}(x) \cap N_{G}(y)\right\}$

(4) $T_{4}(x, y)=N_{G}(y) \backslash\left(T_{1}(x, y) \cup T_{2}(x, y) \cup T_{3}(x, y)\right)$.

The illustrations of $T_{1}(x, y), T_{2}(x, y), T_{3}(x, y)$, and $T_{4}(x, y)$ are shown in Figure 2 (corresponding vertices pointed by dashed arrows).

Theorem 19 (Wang [18], 1996). For any nonempty graph $G$,

$$
b(G) \leqslant \min _{x y \in E(G)}\left\{d_{G}(x)+\left|T_{4}(x, y)\right|\right\} .
$$

The graph in Figure 2 shows that the upper bound given in Theorem 19 is better than that in Theorems 16 and 18, for the upper bounds obtained from these two theorems are $d_{G}(x)+d_{G}(y)-1=11$ and $d_{G}(x)+d_{G}(y)-\left|N_{G}(x) \cap N_{G}(y)\right|=$ 9 , respectively, while the upper bound given by Theorem 19 is $d_{G}(x)+\left|T_{4}(x, y)\right|=6$.

The following result is also an improvement of Theorem 14, in which $t=2$. 
Theorem 20 (Teschner [10], 1997). If $G$ contains a complete subgraph $K_{t}$ with $t \geqslant 2$, then $b(G) \leqslant \min _{x y \in E\left(K_{t}\right)}\left\{d_{G}(x)+\right.$ $\left.d_{G}(y)-t+1\right\}$.

Following Fricke et al. [19], a vertex $x$ of a graph $G$ is $\gamma$ good if $x$ belongs to some $\gamma$-set of $G$ and $\gamma$-bad if $x$ belongs to no $\gamma$-set of $G$. Let $A(G)$ be the set of $\gamma$-good vertices, and let $B(G)$ be the set of $\gamma$-bad vertices in $G$. Clearly, $\{A(G), B(G)\}$ is a partition of $V(G)$. Note there exists some $x \in A$ such that $\gamma(G-x)=\gamma(G)$, say, one end-vertex of $P_{5}$. Samodivkin [20] presented some sharp upper bounds for $b(G)$ in terms of $\gamma$ good and $\gamma$-bad vertices of $G$.

Theorem 21 (Samodivkin [20], 2008). Let G be a graph.

(a) Let $C(G)=\{x \in V(G): \gamma(G-x) \geqslant \gamma(G)\}$. If $C(G) \neq \emptyset$, then

$b(G) \leqslant \min \left\{d_{G}(x)+\gamma(G)-\gamma(G-x): x \in C(G)\right\}$.

(b) If $B \neq \emptyset$, then

$$
b(G) \leqslant \min \left\{\left|N_{G}(x) \cap A\right|: x \in B(G)\right\} .
$$

Theorem 22 (Samodivkin [20], 2008). Let $G$ be a graph. If $A^{+}(G)=\left\{x \in A(G): d_{G}(x) \geqslant 1\right.$ and $\left.\gamma(G-x)<\gamma(G)\right\} \neq \emptyset$, then

$$
b(G) \leqslant \min _{x \in A^{+}(G), y \in B(G-x)}\left\{d_{G}(x)+\left|N_{G}(y) \cap A(G-x)\right|\right\} .
$$

Proposition 23 (Samodivkin [20], 2008). Under the notation of Theorem 19, if $x \in A^{+}(G)$, then $\left(T_{1}(x, y)-\{x\}\right) \cup T_{2}(x, y) \cup$ $T_{3}(x, y) \subseteq N_{G}(y) \backslash B(G-x)$.

By Proposition 23, if $x \in A^{+}(G)$, then

$$
\begin{aligned}
& d_{G}(x)+\min _{y \in N_{G}(x)}\left\{\left|T_{4}(x, y)\right|\right\} \\
& \geqslant d_{G}(x)+\min _{y \in N_{G}(x)}\left\{\left|N_{G}(y) \cap A(G-x)\right|\right\} \\
& \geqslant d_{G}(x)+\min _{y \in B(G-x)}\left\{\left|N_{G}(y) \cap A(G-x)\right|\right\} .
\end{aligned}
$$

Hence, Theorem 19 can be seen to follow from Theorem 22 . Any graph $G$ with $b(G)$ achieving the upper bound in Theorem 19 can be used to show that the bound in Theorem 22 is sharp.

Let $t \geqslant 2$ be an integer. Samodivkin [20] constructed a very interesting graph $G_{t}$ to show that the upper bound in Theorem 22 is better than the known bounds. Let $H_{0}, H_{1}, H_{2}, \ldots, H_{t+1}$ be mutually vertex-disjoint graphs such that $H_{0} \cong K_{2}, H_{t+1} \cong K_{t+3}$ and $H_{i} \cong K_{t+3}-e$ for each $i=1,2, \ldots, t$. Let $V\left(H_{0}\right)=\{x, y\}, x_{t+1} \in V\left(H_{t+1}\right)$ and

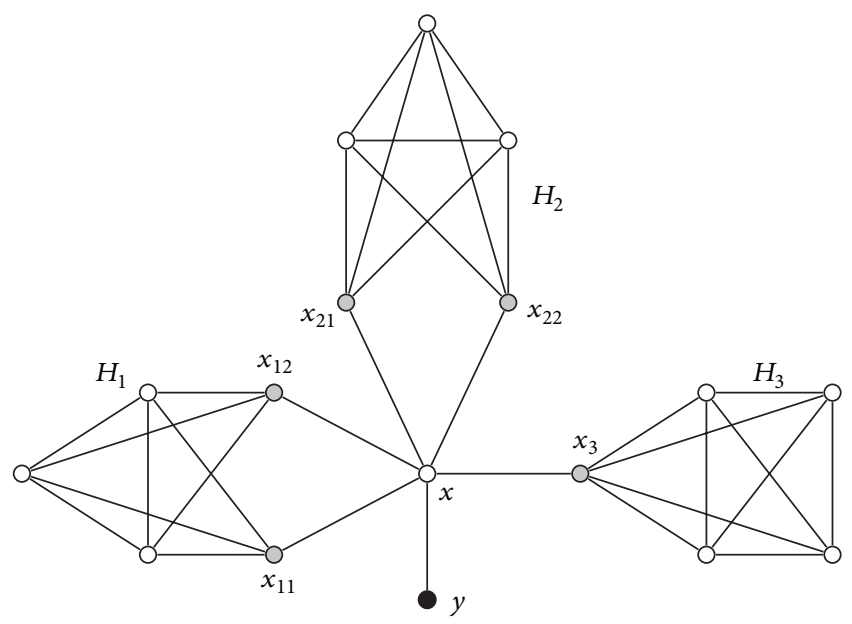

FIgURE 3: The graph $G_{2}$.

$x_{i 1}, x_{i 2} \in V\left(H_{i}\right), x_{i 1} x_{i 2} \notin E\left(H_{i}\right)$ for each $i=1,2, \ldots, t$. The graph $G_{t}$ is defined as follows:

$$
\begin{gathered}
V\left(G_{t}\right)=\bigcup_{k=0}^{t+1} V\left(H_{k}\right), \\
E\left(G_{t}\right)=\left(\bigcup_{k=0}^{t+1} E\left(H_{k}\right)\right) \cup\left(\bigcup_{i=1}^{t}\left\{x x_{i 1}, x x_{i 2}\right\}\right) \cup\left\{x x_{t+1}\right\} .
\end{gathered}
$$

Such a constructed graph $G_{t}$ is shown in Figure 3 when $t=2$.

Observe that $\gamma\left(G_{t}\right)=t+2, A\left(G_{t}\right)=V\left(G_{t}\right), d_{G_{t}}(x)=$ $2 t+2, d_{G_{t}}\left(x_{t+1}\right)=t+3, d_{G_{t}}(y)=1$, and $d_{G_{t}}(z)=t+2$ for each $z \in V\left(G_{t}-\left\{x, y, x_{t+1}\right\}\right)$. Moreover, $\gamma(G-y)<\gamma(G)$ and $\gamma\left(G_{t}-z\right)=\gamma\left(G_{t}\right)$ for any $z \in V\left(G_{t}\right)-\{y\}$. Hence, each of the bounds stated in Theorems 13-20 is greater than or equals $t+2$.

Consider the graph $G_{t}-x y$. Clearly, $\gamma\left(G_{t}-x y\right)=\gamma\left(G_{t}\right)$ and

$$
\begin{aligned}
B\left(G_{t}-x y\right) & =B\left(G_{t}-y\right) \\
& =\{x\} \cup V\left(H_{t+1}-x_{t+1}\right) \cup\left(\bigcup_{k=1}^{t}\left\{x_{k_{1}}, x_{k_{2}}\right\}\right) .
\end{aligned}
$$

Therefore, $N_{G_{t}}(x) \cap G\left(G_{t}-y\right)=\left\{x_{t+1}\right\}$ which implies that the upper bound stated in Theorem 22 is equal to $d_{G_{t}}(y)+$ $\left|\left\{x_{t+1}\right\}\right|=2$. Clearly, $b\left(G_{t}\right)=2$, and hence this bound is sharp for $G_{t}$.

From the graph $G_{t}$, we obtain the following statement immediately.

Proposition 24. For every integer $t \geqslant 2$, there is a graph $G$ such that the difference between any upper bound stated in Theorems 13-20 and the upper bound in Theorem 22 is equal to $t$.

Although Theorem 22 supplies us with the upper bound that is closer to $b(G)$ for some graph $G$ than what any one of 
Theorems 13-20 provides, it is not easy to determine the sets $A^{+}(G)$ and $B(G)$ mentioned in Theorem 22 for an arbitrary graph $G$. Thus, the upper bound given in Theorem 22 is of theoretical importance, but not applied since, until now, we have not found a new class of graphs whose bondage numbers are determined by Theorem 22 .

The above-mentioned upper bounds on the bondage number are involved in only degrees of two vertices. Hartnell and Rall [11] established an upper bound on $b(G)$ in terms of the numbers of vertices and edges of $G$ with order $n$. For any connected graph $G$, let $\bar{\delta}(G)$ represent the average degree of vertices in $G$; that is, $\bar{\delta}(G)=(1 / n) \sum_{x \in V} d_{G}(x)$. Hartnell and Rall first discovered the following proposition.

Proposition 25. For any connected graph $G$, there exist two vertices $x$ and $y$ with distance at most two and with the property that $d_{G}(x)+d_{G}(y) \leqslant 2 \bar{\delta}(G)$.

Using Proposition 25 and Theorem 16, Hartnell and Rall gave the following bound.

Theorem 26 (Hartnell and Rall [11], 1999). $b(G) \leqslant 2 \bar{\delta}(G)-1$ for any connected graph $G$.

Note that $2 \varepsilon=n \bar{\delta}(G)$ for any graph $G$ with order $n$ and size $\varepsilon$. Theorem 26 implies the following bound in terms of order $n$ and size $\varepsilon$.

Corollary 27. $b(G) \leqslant(4 \varepsilon / n)-1$ for any connected graph $G$ with order $n$ and size $\varepsilon$.

A lower bound on $\varepsilon$ in terms of order $n$ and the bondage number is obtained from Corollary 28 immediately.

Corollary 28. $\varepsilon \geqslant(n / 4)(b(G)+1)$ for any connected graph $G$ with order $n$ and size $\varepsilon$.

Hartnell and Rall [11] gave some graphs $G$ with order $n$ to show that for each value of $b(G)$; the lower bound on $\varepsilon(G)$ given in the Corollary 28 is sharp for some values of $n$.

3.2. Bounds Implied by Connectivity. Use $\kappa(G)$ and $\lambda(G)$ to denote the vertex-connectivity and the edge-connectivity of a connected graph $G$, respectively, which are the minimum numbers of vertices and edges whose removal result in $G$ disconnected. The famous Whitney inequality is stated as $\kappa(G) \leqslant \lambda(G) \leqslant \delta(G)$ for any graph or digraph $G$. Corollary 15 is improved by several authors as follows.

Theorem 29 (Hartnell and Rall [17], 1994; Teschner [10], 1997). If $G$ is a connected graph, then $b(G) \leqslant \lambda(G)+\Delta(G)-1$.

The upper bound given in Theorem 29 can be attained. For example, a cycle $C_{3 k+1}$ with $k \geqslant 1, b\left(C_{3 k+1}\right)=3$ by Theorem 1. Since $\kappa\left(C_{3 k+1}\right)=\lambda\left(C_{3 k+1}\right)=2$, we have $\kappa\left(C_{3 k+1}\right)+$ $\lambda\left(C_{3 k+1}\right)-1=2+2-1=3=b\left(C_{3 k+1}\right)$.

Motivated by Corollary 15, Theorems 29, and the Whitney inequality, Dunbar et al. [21] naturally proposed the following conjecture.

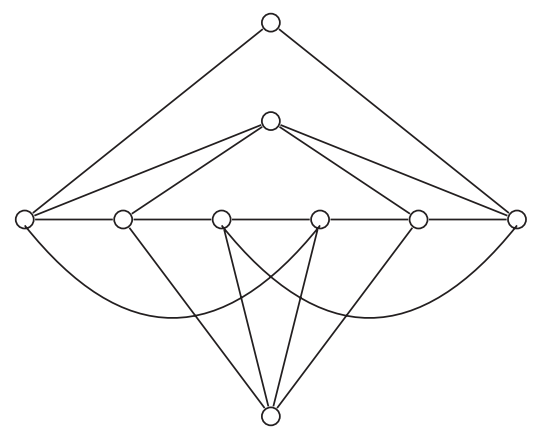

Figure 4: A graph $H$ with $\gamma(H)=3$ and $b(H)=5$.

Conjecture 30. If $G$ is a connected graph, then $b(G) \leqslant \Delta(G)+$ $\kappa(G)-1$.

However, Liu and Sun [22] presented a counterexample to this conjecture. They first constructed a graph $H$ showed in Figure 4 with $\gamma(H)=3$ and $b(H)=5$. Then, let $G$ be the disjoint union of two copies of $H$ by identifying two vertices of degree two. They proved that $b(G) \geqslant 5$. Clearly, $G$ is a 4 regular graph with $\kappa(G)=1$ and $\lambda(G)=2$, and so $b(G) \leqslant 5$ by Theorem 29. Thus, $b(G)=5>4=\Delta(G)+\kappa(G)-1$.

With a suspicion of the relationship between the bondage number and the vertex-connectivity of a graph, the following conjecture is proposed.

Conjecture 31 (Liu and Sun [22], 2003). For any positive integer $r$, there exists a connected graph $G$ such that $b(G) \geqslant$ $\Delta(G)+\kappa(G)+r$.

To the knowledge of the author, until now no results have been known about this conjecture.

We conclude this subsection with the following remarks. From Theorem 29, if Conjecture 31 holds for some connected graph $G$, then $\lambda(G)>\kappa(G)+r$, which implies that $G$ is of large edge-connectivity and small vertex-connectivity. Use $\xi(G)$ to denote the minimum edge-degree of $G$; that is,

$$
\xi(G)=\min _{x y \in E(G)}\left\{d_{G}(x)+d_{G}(y)-2\right\} .
$$

Theorem 14 implies the following result.

Proposition 32. $b(G) \leqslant \xi(G)+1$ for any graph $G$.

Use $\lambda^{\prime}(G)$ to denote the restricted edge-connectivity of a connected graph $G$, which is the minimum number of edges whose removal result in $G$ disconnected and no isolated vertices.

Proposition 33 (Esfahanian and Hakimi [23], 1988). If $G$ is neither $K_{1, n}$ nor $K_{3}$, then $\lambda^{\prime}(G) \leqslant \xi(G)$.

Combining Propositions 32 and 33, we propose a conjecture.

Conjecture 34. If a connected $G$ is neither $K_{1, n}$ nor $K_{3}$, then $b(G) \leqslant \delta(G)+\lambda^{\prime}(G)-1$. 
A cycle $C_{3 k+1}$ also satisfies $b\left(C_{3 k+1}\right)=3=\delta\left(C_{3 k+1}\right)+$ $\lambda^{\prime}\left(C_{3 k+1}\right)-1$ since $\lambda^{\prime}\left(C_{3 k+1}\right)=\delta\left(C_{3 k+1}\right)=2$ for any integer $k \geqslant 1$.

For the graph $H$ shown in Figure $4, \lambda^{\prime}(H)=4$ and $\delta(H)=$ 2 , and so $b(H)=5=\delta(H)+\lambda^{\prime}(H)-1$.

For the 4-regular graph $G$ constructed by Liu and Sun [22] obtained from the disjoint union of two copies of the graph $H$ showed in Figure 4 by identifying two vertices of degree two, we have $b(G) \geqslant 5$. Clearly, $\lambda^{\prime}(G)=2$. Thus, $b(G)=5=$ $\delta(G)+\lambda^{\prime}(G)-1$.

For the 4-regular graph $G_{t}$ constructed by Samodivkin [20], see Figure 3 for $t=2, b\left(G_{t}\right)=2$. Clearly, $\delta\left(G_{t}\right)=1$ and $\lambda^{\prime}(G)=2$. Thus, $b(G)=2=\delta(G)+\lambda^{\prime}(G)-1$.

These examples show that if Conjecture 34 is true then the given upper bound is tight.

3.3. Bounds Implied by Degree Sequence. Now let us return to Theorem 16, from which Teschner [10] obtained some other bounds in terms of the degree sequences. The degree sequence $\pi(G)$ of a graph $G$ with vertex-set $V=\left\{x_{1}, x_{2}, \ldots, x_{n}\right\}$ is the sequence $\pi=\left(d_{1}, d_{2}, \ldots, d_{n}\right)$ with $d_{1} \leqslant d_{2} \leqslant \cdots \leqslant d_{n}$, where $d_{i}=d_{G}\left(x_{i}\right)$ for each $i=1,2, \ldots, n$. The following result is essentially a corollary of Theorem 16 .

Theorem 35 (Teschner [10], 1997). Let $G$ be a nonempty graph with degree sequence $\pi(G)$. If $\alpha_{2}(G)=t$, then $b(G) \leqslant d_{t}+d_{t+1}-$ 1 .

Combining Theorem 35 with Proposition 10 (i.e., $\alpha_{2}(G) \leqslant$ $\gamma(G)$ ), we have the following corollary.

Corollary 36 (Teschner [10], 1997). Let $G$ be a nonempty graph with the degree sequence $\pi(G)$. If $\gamma(G)=\gamma$, then $b(G) \leqslant$ $d_{\gamma}+d_{\gamma+1}-1$.

In [10], Teschner showed that these two bounds are sharp for arbitrarily many graphs. Let $H=C_{3 k+1}+\left\{x_{1} x_{4}, x_{1} x_{3 k-1}\right\}$, where $C_{3 k+1}$ is a cycle $\left(x_{1}, x_{2}, \ldots, x_{3 k+1}, x_{1}\right)$ for any integer $k \geqslant 2$. Then $\gamma(H)=k+1$ and so $b(H) \leqslant 2+2-1=3$ by Corollary 36. Since $C_{3 k+1}$ is a spanning subgraph of $H$ and $\gamma(G)=\gamma(H)$, Observation 1 yields that $b(H) \geqslant b\left(C_{3 k+1}\right)=3$, and so $b(H)=3$.

Although various upper bounds have been established as the above, we find that the appearance of these bounds is essentially based upon the local structures of a graph, precisely speaking, the structures of the neighborhoods of two vertices within distance 2 . Even if these bounds can be achieved by some special graphs, it is more often not the case. The reason lies essentially in the definition of the bondage number, which is the minimum value among all bondage sets, an integral property of a graph. While it easy to find upper bounds just by choosing some bondage set, the gap between the exact value of the bondage number and such a bound obtained only from local structures of a graph is often large. For example, a star $K_{1, \Delta}$, however large $\Delta$ is, $b\left(K_{1, \Delta}\right)=1$. Therefore, one has been longing for better bounds upon some integral parameters. However, as what we will see below, it is difficult to establish such upper bounds.
3.4. Bounds in $\gamma$-Critical Graphs. A graph $G$ is called a vertex domination-critical graph (vc-graph or $\gamma$-critical for short) if $\gamma(G-x)<\gamma(G)$ for any $x \in V(G)$, proposed by Brigham et al. [24] in 1988. Several families of graphs are known to be $\gamma$-critical. From definition, it is clear that if $G$ is a $\gamma$-critical graph, then $\gamma(G) \geqslant 2$. The class of $\gamma$-critical graphs with $\gamma=2$ is characterized as follows.

Proposition 37 (Brigham et al. [24], 1988). A graph $G$ with $\gamma(G)=2$ is a $\gamma$-critical graph if and only if $G$ is a complete graph $K_{2 t}(t \geqslant 2)$ with a perfect matching removed.

A more interesting family is composed of the $n$-critical graphs $G_{m, n}$ defined for $m, n \geqslant 2$ by the circulant undirected graph $G(N, \pm S)$, where $N=(m+1)(n-1)+1$ and $S=\{1,2, \ldots,\lfloor m / 2\rfloor\}$, in which $V(G(N, \pm S))=N$ and $E(G(N, \pm S))=\{i j:|j-i| \equiv s(\bmod N), s \in S\}$.

The reason why $\gamma$-critical graphs are of special interest in this context is easy to see that they play an important role in study of the bondage number. For instance, it immediately follows from Theorem 13 that if $b(G)>\Delta(G)$ then $G$ is a $\gamma$ critical graph. The $\gamma$-critical graphs are defined exactly in this way. In order to find graphs $G$ with a high bondage number (i.e., higher than $\Delta(G)$ ) and beyond its general upper bounds for the bondage number, we, therefore, have to look at $\gamma$ critical graphs.

The bondage numbers of some $\gamma$-critical graphs have been examined by several authors; see, for example, [10, 20, 25, 26]. From Theorem 13, we know that the bondage number of a graph $G$ is bounded from above by $\Delta(G)$ if $G$ is not a $\gamma$-critical graph. For $\gamma$-critical graphs, it is more difficult to find an upper bound. We will see that the bondage numbers of $\gamma$-critical graphs in general are not even bounded from above by $\Delta+c$ for any fixed natural number $c$.

In this subsection, we introduce some upper bounds for the bondage number of a $\gamma$-critical graph. By Proposition 37, we easily obtain the following result.

Theorem 38. If $G$ is a $\gamma$-critical graph with $\gamma(G)=2$, then $b(G) \leqslant \Delta+1$.

In Section 4, by Theorem 59, we will see that the equality in Theorem 38 holds; that is, $b(G)=\Delta+1$ if $G$ is a $\gamma$-critical graph with $\gamma(G)=2$.

Theorem 39 (Teschner [10], 1997). Let $G$ be a $\gamma$-critical graph with degree sequence $\pi(G)$. Then $b(G) \leqslant \max \left\{\Delta(G)+1, d_{1}+\right.$ $\left.d_{2}+\cdots+d_{\gamma-1}\right\}$, where $\gamma=\gamma(G)$.

As we mentioned above, if $G$ is a $\gamma$-critical graph with $\gamma(G)=2$, then $b(G)=\Delta+1$, which shows that the bound given in Theorem 39 can be attained for $\gamma=2$. However, we have not known whether this bound is tight for general $\gamma \geqslant 3$. Theorem 39 gives the following corollary immediately.

Corollary 40 (Teschner [10], 1997). Let $G$ be a $\gamma$-critical graph with degree sequence $\pi(G)$. If $\gamma(G)=3$, then $b(G) \leqslant$ $\max \left\{\Delta(G)+1, \delta(G)+d_{2}\right\}$. 
From Theorem 38 and Corollary 40, we have $b(G) \leqslant$ $2 \Delta(G)$ if $G$ is a $\gamma$-critical graph with $\gamma(G) \leqslant 3$. The following result shows that this bound is not tight.

Theorem 41 (Teschner [26], 1995). Let $G$ be a $\gamma$-critical graph graph with $\gamma(G) \leqslant 3$. Then $b(G) \leqslant(3 / 2) \Delta(G)$.

Until now, we have not known whether the bound given in Theorem 41 is tight or not. We state two conjectures on $\gamma$-critical graphs proposed by Samodivkin [20]. The first of them is motivated by Theorems 22 and 19.

Conjecture 42 (Samodivkin [20], 2008). For every connected nontrivial $\gamma$-critical graph $G$,

$$
\min _{x \in A^{+}(G), y \in B(G-x)}\left\{d_{G}(x)+\left|N_{G}(y) \cap A(G-x)\right|\right\} \leqslant \frac{3}{2} \Delta(G) .
$$

To state the second conjecture, we need the following result on $\gamma$-critical graphs.

Proposition 43. If $G$ is a $\gamma$-critical graph, then $v(G) \leqslant(\Delta(G)+$ $1)(\gamma(G)-1)+1$; moreover, if the equality holds, then $G$ is regular.

The upper bound in Proposition 43 is sharp in the sense that equality holds for the infinite class of $\gamma$-critical graphs $G_{m, n}$ defined in the beginning of this subsection. In Proposition 43, the first result is due to Brigham et al. [24] in 1988; the second is due to Fulman et al. [27] in 1995.

For a $\gamma$-critical graph $G$ with $\gamma=3$, by Proposition 43, $v(G) \leqslant 2 \Delta(G)+3$.

Theorem 44 (Teschner [26], 1995). If $G$ is a $\gamma$-critical graph with $\gamma=3$ and $v(G)=2 \Delta(G)+3$, then $b(G) \leqslant \Delta+1$.

We have not known yet whether the equality in Theorem 44 holds or not. However, Samodivkin proposed the following conjecture.

Conjecture 45 (Samodivkin [20], 2008). If $G$ is a $\gamma$-critical graph with $(\Delta(G)+1)(\gamma-1)+1$ vertices, then $b(G)=\Delta(G)+1$.

In general, based on Theorem 41, Teschner proposed the following conjecture.

Conjecture 46 (Teschner [26], 1995). If $\mathrm{T}$ is a $\gamma$-critical graph, then $b(G) \leqslant(3 / 2) \Delta(G)$ for $\gamma \geqslant 4$.

We conclude this subsection with some remarks. Graphs which are minimal or critical with respect to a given property or parameter frequently play an important role in the investigation of that property or parameter. Not only are such graphs of considerable interest in their own right, but also a knowledge of their structure often aids in the development of the general theory. In particular, when investigating any finite structure, a great number of results are proven by induction. Consequently, it is desirable to learn as much as possible about those graphs that are critical with respect to a given property or parameter so as to aid and abet such investigations.
In this subsection, we survey some results on the bondage number for $\gamma$-critical graphs. Although these results are not very perfect, they provides a feasible method to approach the bondage number from different viewpoints. In particular, the methods given in Teschner [26] worth further exploration and development.

The following proposition is maybe useful for us to further investigate the bondage number of a $\gamma$-critical graph.

Proposition 47 (Brigham et al. [24], 1988). If $G$ has a nonisolated vertex $x$ such that the subgraph induced by $N_{G}(x)$ is a complete graph, then $G$ is not $\gamma$-critical.

This simple fact shows that any $\gamma$-critical graph contains no vertices of degree one.

3.5. Bounds Implied by Domination. In the preceding subsection, we introduced some upper bounds on the bondage numbers for $\gamma$-critical graphs by consideration of dominations. In this subsection, we introduce related results for a general graph with given domination number.

Theorem 48 (Fink et al. [8], 1990). For any connected graph G of $\operatorname{order} n(\geqslant 2)$,

(a) $b(G) \leqslant n-1$;

(b) $b(G) \leqslant \Delta(G)+1$ if $\gamma(G)=2$;

(c) $b(G) \leqslant n-\gamma(G)+1$.

While the upper bound of $n-1$ on $b(G)$ is not particularly good for many classes of graphs (e.g., trees and cycles), it is an attainable bound. For example, if $G$ is a complete $t$-partite graph $K_{t}(2)$ for $t=n / 2$ and $n \leqslant 4$ an even integer, then the three bounds on $b(G)$ in Theorem 48 are sharp.

Teschner [26] consider a graph with $\gamma(G)=1$ or $\gamma(G)=2$. The next result is almost trivial but useful.

Lemma 49. Let $G$ be a graph with order $n$ and $\gamma(G)=1, t$ the number of vertices of degree $n-1$. Then $b(G)=\lceil t / 2\rceil$.

Since $t \leqslant \Delta(G)$ clearly, Lemma 49 yields the following result immediately.

Theorem 50 (Teschner [26], 1995). $b(G) \leqslant(1 / 2) \Delta+1$ for any graph $G$ with $\gamma(G)=1$.

For a complete graph $K_{2 k+1}, b\left(K_{2 k+1}\right)=k+1=(1 / 2) \Delta+1$, which shows that the upper bound given in Theorem 50 can be attained. For a graph $G$ with $\gamma(G)=2$, by Theorem 59 later, the upper bound given in Theorem 48 (b) can be also attained by a 2 -critical graph (see Proposition 37 ).

3.6. Two Conjectures. In 1990, when Fink et al. [8] introduced the concept of the bondage number; they proposed the following conjecture.

Conjecture 51. $b(G) \leqslant \Delta(G)+1$ for any nonempty graph $G$. 
Although some results partially support Conjecture 51, Teschner [28] in 1993 found a counterexample to this conjecture, the cartesian product $K_{3} \times K_{3}$, which shows that $b(G)=\Delta(G)+2$.

If a graph $G$ is a counterexample to Conjecture 51, it must be a $\gamma$-critical graph by Theorem 13. That is why the vertex domination-critical graphs are of special interest in the literature.

Now, we return to Conjecture 51. Hartnell and Rall [17] and Teschner [29], independently, proved that $b(G)$ can be much greater than $\Delta(G)$ by showing the following result.

Theorem 52 (Hartnell and Rall [17], 1994; Teschner [29]. 1996). For an integer $n \geqslant 3$, let $G_{n}$ be the cartesian product $K_{n} \times K_{n}$. Then $b\left(G_{n}\right)=3(n-1)=(3 / 2) \Delta\left(G_{n}\right)$.

This theorem shows that there exist no upper bounds of the form $b(G) \leqslant \Delta(G)+c$ for any integer $c$. Teschner [26] proved that $b(G) \leqslant(3 / 2) \Delta(G)$ for any graph $G$ with $\gamma(G) \leqslant 2$ (see Theorems 50 and 48) and for $\gamma$-critical graphs with $\gamma(G)=3$ and proposed the following conjecture.

Conjecture 53 (Teschner [26], 1995). $b(G) \leqslant(3 / 2) \Delta(G)$ for any graph $G$.

We believe that this conjecture is true, but so far no much work about it has been known.

\section{Lower Bounds}

Since the bondage number is defined as the smallest number of edges whose removal results in an increase of domination number, each constructive method that creates a concrete bondage set leads to an upper bound on the bondage number. For that reason, it is hard to find lower bounds. Nevertheless, there are still a few lower bounds.

4.1. Bounds Implied by Subgraphs. The first lower bound on the bondage number is gotten in terms of its spanning subgraph.

Theorem 54 (Teschner [10], 1997). Let $H$ be a spanning subgraph of a nonempty graph $G$. If $\gamma(H)=\gamma(G)$, then $b(H) \leqslant$ $b(G)$.

By Theorem $1, b\left(C_{n}\right)=3$ if $n \equiv 1(\bmod 3)$ and $b\left(C_{n}\right)=$ 2 , otherwise $b\left(P_{n}\right)=2$ if $n \equiv 1(\bmod 3)$ and $b\left(P_{n}\right)=1$ otherwise. From these results and Theorem 54, we get the following two corollaries.

Corollary 55. If $G$ is hamiltonian with order $n \geqslant 3$ and $\gamma(G)=$ $\lceil n / 3\rceil$, then $b(G) \geqslant 2$ and in addition $b(G) \geqslant 3$ if $n \equiv 1(\mathrm{mod}$ 3).

Corollary 56. If $G$ with order $n \geqslant 2$ has a hamiltonian path and $\gamma(G)=\lceil n / 3\rceil$, then $b(G) \geqslant 2$ if $n \equiv 1(\bmod 3)$.

4.2. Other Bounds. The vertex covering number $\beta(G)$ of $G$ is the minimum number of vertices that are incident with all

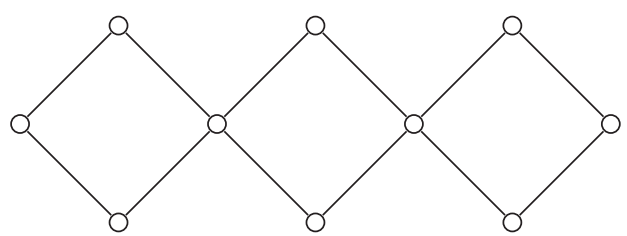

FigURE 5: A $\gamma$-critical graph with $\beta=\gamma=4, \delta=2$, and $b=3$.

edges in $G$. If $G$ has no isolated vertices, then $\gamma(G) \leqslant \beta(G)$ clearly. In [30], Volkmann gave a lot of graphs with $\beta=\gamma$.

Theorem 57 (Teschner [10], 1997). Let $G$ be a graph. If $\gamma(G)=$ $\beta(G)$, then

(a) $b(G) \geqslant \delta(G)$;

(b) $b(G) \geqslant \delta(G)+1$ if $G$ is a $\gamma$-critical graph.

The graph shown in Figure 5 shows that the bound given in Theorem 57(b) is sharp. For the graph $G$, it is a $\gamma$-critical graph and $\gamma(G)=\beta(G)=4$. By Theorem 57, we have $b(G) \geqslant$ 3 . On the other hand, $b(G) \leqslant 3$ by Theorem 16 . Thus, $b(G)=$ 3.

Proposition 58 (Sanchis [31], 1991). Let $G$ be a graph $G$ of order $n(\geqslant 6)$ and size $\varepsilon$. If $G$ has no isolated vertices and $3 \leqslant \gamma(G) \leqslant n / 2$, then $\varepsilon \leqslant\left(\begin{array}{c}n-\gamma(G)+1 \\ 2\end{array}\right)$.

Using the idea in the proof of Theorem 57, every upper bound for $\gamma(G)$ can lead to a lower bound for $b(G)$. In this way, Teschner [10] obtained another lower bound from Proposition 58.

Theorem 59 (Teschner [10], 1997). Let $G$ be a graph $G$ of order $n(\geqslant 6)$ and size $\varepsilon$. If $2 \leqslant \gamma(G) \leqslant n / 2-1$, then

(a) $b(G) \geqslant \min \left\{\delta(G), \varepsilon-\left(\begin{array}{c}n-\gamma(G) \\ 2\end{array}\right)\right\}$

(b) $b(G) \geqslant \min \left\{\delta(G)+1, \varepsilon-\left(\begin{array}{c}n-\gamma(G) \\ 2\end{array}\right)\right\}$ if $G$ is a $\gamma$-critical graph.

The lower bound in Theorem 59(b) is sharp for a class of $\gamma$-critical graphs with domination number 2. Let $G$ be the graph obtained from complete graph $K_{2 t}(t \geqslant 2)$ by removing a perfect matching. By Proposition 37, $G$ is a $\gamma$-critical graph with $\gamma(G)=2$. Then $b(G) \geqslant \delta(G)+1=\Delta(G)+1$ by Theorem 59 and $b(G) \leqslant \Delta(G)+1$ by Theorem 38 , and so $b(G)=\Delta(G)+1=2 t-1$.

As far as the author knows, there are no more lower bounds in the present literature. In view of applications of the bondage number, a network is vulnerable if its bondage number is small while it is stable if its bondage number is large. Therefore, better lower bounds let us learn better about the stability of networks from this point of view. Thus, it is of great significance to seek more lower bounds for various classes of graphs. 


\section{Results on Graph Operations}

Generally speaking, it is quite difficult to determine the exact value of the bondage number for a given graph since it strongly depends on the dominating number of the graph. Thus, determining bondage numbers for some special graphs is interesting if the dominating numbers of those graphs are known or can be easily determined. In this section, we will introduce some known results on bondage numbers for some special classes of graphs obtained by some graph operations.

5.1. Cartesian Product Graphs. Let $G_{1}=\left(V_{1}, E_{1}\right)$ and $G_{2}=$ $\left(V_{2}, E_{2}\right)$ be two graphs. The Cartesian product of $G_{1}$ and $G_{2}$ is an undirected graph, denoted by $G_{1} \times G_{2}$, where $V\left(G_{1} \times\right.$ $\left.G_{2}\right)=V_{1} \times V_{2}$; two distinct vertices $x_{1} x_{2}$ and $y_{1} y_{2}$, where $x_{1}, y_{1} \in V\left(G_{1}\right)$ and $x_{2}, y_{2} \in V\left(G_{2}\right)$, are linked by an edge in $G_{1} \times G_{2}$ if and only if either $x_{1}=y_{1}$ and $x_{2} y_{2} \in E\left(G_{2}\right)$, or $x_{2}=y_{2}$ and $x_{1} y_{1} \in E\left(G_{1}\right)$. The Cartesian product is a very effective method for constructing a larger graph from several specified small graphs.

Theorem 60 (Dunbar et al. [21], 1998). For $n \geqslant 3$,

$$
b\left(C_{n} \times K_{2}\right)= \begin{cases}2 & \text { if } n \equiv 0 \text { or } 1(\bmod 4), \\ 3 & \text { if } n \equiv 3(\bmod 4), \\ 4 & \text { if } n \equiv 2(\bmod 4) .\end{cases}
$$

For the Cartesian product $C_{n} \times C_{m}$ of two cycles $C_{n}$ and $C_{m}$, where $n \geqslant 4$ and $m \geqslant 3$, Klavžar and Seifter [32] determined $\gamma\left(C_{n} \times C_{m}\right)$ for some $n$ and $m$. For example, $\gamma\left(C_{n} \times C_{4}\right)=n$ for $n \geqslant 3$ and $\gamma\left(C_{n} \times C_{3}\right)=n-\lfloor n / 4\rfloor$ for $n \geqslant 4$. Kim [33] determined $b\left(C_{3} \times C_{3}\right)=6$ and $b\left(C_{4} \times C_{4}\right)=4$. For a general $n \geqslant 4$, the exact values of the bondage numbers of $C_{n} \times C_{3}$ and $C_{n} \times C_{4}$ are determined as follows.

Theorem 61 (Sohn et al. [34], 2007). For $n \geqslant 4$,

$$
b\left(C_{n} \times C_{3}\right)= \begin{cases}2 & \text { if } n \equiv 0(\bmod 4), \\ 4 & \text { if } n \equiv 1 \operatorname{or} 2(\bmod 4), \\ 5 & \text { if } n \equiv 3(\bmod 4)\end{cases}
$$

Theorem 62 (Kang et al. [35], 2005). $b\left(C_{n} \times C_{4}\right)=4$ for $n \geqslant 4$.

For larger $m$ and $n$, Huang and $\mathrm{Xu}$ [36] obtained the following result; see Theorem 197 for more details.

Theorem 63 (Huang and $\mathrm{Xu}[36], 2008) . b\left(C_{5 i} \times C_{5 j}\right)=3$ for any positive integers $i$ and $j$.

Xiang et al. [37] determined that for $n \geqslant 5$,

$$
b\left(C_{n} \times C_{5}\right) \begin{cases}=3 & \text { if } n \equiv 0 \text { or } 1(\bmod 5) \\ =4 & \text { if } n \equiv 2 \operatorname{or} 4(\bmod 5) \\ \leqslant 7 & \text { if } n \equiv 3(\bmod 5) .\end{cases}
$$

For the Cartesian product $P_{n} \times P_{m}$ of two paths $P_{n}$ and $P_{m}$, Jacobson and Kinch [38] determined $\gamma\left(P_{n} \times P_{2}\right)=\lceil(n+1) / 2\rceil$, $\gamma\left(P_{n} \times P_{3}\right)=n-\lfloor(n-1) / 4\rfloor$, and $\gamma\left(P_{n} \times P_{4}\right)=n+1$ if $n=$ $1,2,3,5,6,9$, and $n$ otherwise. The bondage number of $P_{n} \times P_{m}$ for $n \geqslant 4$ and $2 \leqslant m \leqslant 4$ is determined as follows (Li [39] also determined $\left.b\left(P_{n} \times P_{2}\right)\right)$.

Theorem 64 (Hu and $\mathrm{Xu}[40], 2012)$. For $n \geqslant 4$,

$$
\begin{aligned}
& b\left(P_{n} \times P_{2}\right)= \begin{cases}1, & \text { if } n \equiv 1(\bmod 2) \\
2, & \text { if } n \equiv 0(\bmod 2) ;\end{cases} \\
& b\left(P_{n} \times P_{3}\right)= \begin{cases}1, & \text { if } n \equiv 1 \text { or } 2(\bmod 4) \\
2, & \text { if } n \equiv 0 \text { or } 3(\bmod 4),\end{cases} \\
& b\left(P_{n} \times P_{4}\right)=1 \quad \text { for } n \geqslant 14 .
\end{aligned}
$$

From the proof of Theorem 64, we find that if $P_{n}=$ $\{0,1, \ldots, n-1\}$ and $P_{m}=\{0,1, \ldots, m-1\}$, then the removal of the vertex $(0,0)$ in $P_{n} \times P_{m}$ does not change the domination number. If $m$ increases, the effect of $(0,0)$ for the domination number will be smaller and smaller from the probability. Therefore, we expect it is possible that $\gamma\left(P_{n} \times P_{m}-(0,0)\right)=$ $\gamma\left(P_{n} \times P_{m}\right)$ for $m \geqslant 5$ and give the following conjecture.

Conjecture 65. $b\left(P_{n} \times P_{m}\right) \leqslant 2$ for $m \geqslant 5$ and $n \geqslant 4$.

5.2. Block Graphs and Cactus Graphs. In this subsection, we introduce some results for corona graphs, block graphs, and cactus graphs.

The corona $G_{1} \circ G_{2}$, proposed by Frucht and Harary [41], is the graph formed from a copy of $G_{1}$ and $v\left(G_{1}\right)$ copies of $G_{2}$ by joining the $i$ th vertex of $G_{1}$ to the $i$ th copy of $G_{2}$. In particular, we are concerned with the corona $H \circ K_{1}$, the graph formed by adding a new vertex $v_{i}$, and a new edge $u_{i} v_{i}$ for every vertex $u_{i}$ in $H$.

Theorem 66 (Carlson and Develin [42], 2006). $b\left(H \circ K_{1}\right)=$ $\delta(H)+1$.

This is a very important result, which is often used to construct a graph with required bondage number. In other words, this result implies that for any given positive integer $b$ there is a graph $G$ such that $b(G)=b$.

A block graph is a graph whose blocks are complete graphs. Each block in a cactus graph is either a cycle or a $K_{2}$. If each block of a graph is either a complete graph or a cycle, then we call this graph a block-cactus graph.

Theorem 67 (Teschner [43], 1997). $b(G) \leq \Delta(G)$ for any block graph $G$.

Teschner [43] characterized all block graphs with $b(G)=$ $\Delta(G)$. In the same paper, Teschner found that $\gamma$-critical graphs are instrumental in determining bounds for the bondage number of cactus and block graphs and obtained the following result.

Theorem 68 (Teschner [43], 1997). $b(G) \leqslant 3$ for any nontrivial cactus graph $G$.

This bound can be achieved by $H \circ K_{1}$ where $H$ is a nontrivial cactus graph without vertices of degree one 
by Theorem 66. In 1998, Dunbar et al. [21] proposed the following problem.

Problem 2. Characterize all cactus graphs with bondage number 3.

Some upper bounds for block-cactus graphs were also obtained.

Theorem 69 (Dunbar et al. [21], 1998). Let $G$ be a connected block-cactus graph with at least two blocks. Then $b(G) \leqslant \Delta(G)$.

Theorem 70 (Dunbar et al. [21], 1998). Let G be a connected block-cactus graph which is neither a cactus graph nor a block graph. Then $b(G) \leqslant \Delta(G)-1$.

5.3. Edge-Deletion and Edge-Contraction. In order to obtain further results of the bondage number, we may consider how the bondage number changes under some operations of a graph $G$, which remain the domination number unchanged or preserve some property of $G$, such as planarity. Two simple operations satisfying these requirements are the edgedeletion and the edge-contraction.

Theorem 71 (Huang and $\mathrm{Xu}[44], 2012)$. Let $\mathrm{G}$ be any graph and $e \in E(G)$. Then $b(G-e) \geqslant b(G)-1$. Moreover, $b(G-e) \leqslant$ $b(G)$ if $\gamma(G-e)=\gamma(G)$.

From Theorem $1, b\left(C_{n}-e\right)=b\left(P_{n}\right)=b\left(C_{n}\right)-1$ for any $e$ in $C_{n}$, which shows that the lower bound on $b(G-e)$ is sharp. The upper bound can reach for any graph $G$ with $b(G) \geqslant 2$.

Next, we consider the effect of the edge-contraction on the bondage number. Given a graph $G$, the contraction of $G$ by the edge $e=x y$, denoted by $G / x y$, is the graph obtained from $G$ by contracting two vertices $x$ and $y$ to a new vertex and then deleting all multiedges. It is easy to observe that for any graph $G, \gamma(G)-1 \leqslant \gamma(G / x y) \leqslant \gamma(G)$ for any edge $x y$ of G.

Theorem 72 (Huang and $\mathrm{Xu}[44], 2012$ ). Let $\mathrm{G}$ be any graph and $x y$ be any edge in $G$. If $N_{G}(x) \cap N_{G}(y)=\emptyset$ and $\gamma(G / x y)=$ $\gamma(G)$, then $b(G / x y) \geqslant b(G)$.

We can use examples $C_{n}$ and $K_{n}$ to show that the conditions of Theorem 72 are necessary and the lower bound in Theorem 72 is tight. Clearly, $\gamma\left(C_{n}\right)=\lceil n / 3\rceil$ and $\gamma\left(K_{n}\right)=1$; for any edge $x y, C_{n} / x y=C_{n-1}$ and $K_{n} / x y=K_{n-1}$. By Theorem 1, if $n \equiv 1(\bmod 3)$, then $\gamma\left(C_{n} / x y\right)<\gamma(G)$ and $b\left(C_{n} / x y\right)=2<3=b\left(C_{n}\right)$. Thus, the result in Theorem 72 is generally invalid without the hypothesis $\gamma(G / x y)=\gamma(G)$. Furthermore, the condition $N_{G}(x) \cap N_{G}(y)=\emptyset$ cannot be omitted even if $\gamma(G / x y)=\gamma(G)$, since for odd $n, b\left(K_{n} / x y\right)=$ $\lceil(n-1) / 2\rceil<\lceil n / 2\rceil=b\left(K_{n}\right)$, by Theorem 1 .

On the other hand, $b\left(C_{n} / x y\right)=b\left(C_{n}\right)=2$ if $n \equiv 0(\bmod$ $3)$ and $b\left(K_{n} / x y\right)=b\left(K_{n}\right)$ if $n$ is even, which shows that the equality in $b(G / x y) \geqslant b(G)$ may hold. Thus, the bound in Theorem 72 is tight. However, provided all the conditions, $b(G / x y)$ can be arbitrarily larger than $b(G)$. Given a graph $H$, let $G$ be the graph formed from $H \circ K_{1}$ by adding a new vertex $x$ and joining it to an vertex $y$ of degree one in $H \circ K_{1}$. Then $G / x y=H \circ K_{1}, \gamma(G)=\gamma(G / x y)$, and $N_{G}(x) \cap N_{G}(y)=\emptyset$. But $b(G)=1$ since $\gamma(G-x y)=\gamma(G / x y)+1$, and $b(G / x y)=$ $\delta(H)+1$ by Theorem 66 . The gap between $b(G)$ and $b(G / x y)$ is $\delta(H)$.

\section{Results on Planar Graphs}

From Section 2, we have seen that the bondage number for a tree has been completely solved. Moreover, a linear time algorithm to compute the bondage number of a tree is designed by Hartnell et al. [15]. It is quite natural to consider the bondage number for a planar graph. In this section, we state some results and problems on the bondage number for a planar graph.

6.1. Conjecture on Bondage Number. As mentioned in Section 3, the bondage number can be much larger than the maximum degree. But for a planar graph $G$, the bondage number $b(G)$ cannot exceed $\Delta(G)$ too much. It is clear that $b(G) \leqslant \Delta(G)+4$ by Corollary 15 since $\delta(G) \leqslant 5$ for any planar graph G. In 1998, Dunbar et al. [21] posed the following conjecture.

Conjecture 73. If $G$ is a planar graph, then $b(G) \leqslant \Delta(G)+1$.

Because of its attraction, it immediately became the focus of attention when this conjecture is proposed. In fact, the main aim concerning the research of the bondage number for a planar graph is focused on this conjecture.

It has been mentioned in Theorems 1 and 60 that $b\left(C_{3 k+1}\right)=3=\Delta+1$, and $b\left(C_{4 k+2} \times K_{2}\right)=4=\Delta+1$. It is known that $b\left(K_{6}-M\right)=5=\Delta+1$, where $M$ is a perfect matching of the complete graph $K_{6}$. These examples show that if Conjecture 73 is true, then the upper bound is sharp for $2 \leqslant \Delta \leqslant 4$.

Here, we note that it is sufficient to prove this conjecture for connected planar graphs, since the bondage number of a disconnected graph is simply the minimum of the bondage numbers of its components.

The first paper attacking this conjecture is due to Kang and Yuan [45], which confirmed the conjecture for every connected planar graph $G$ with $\Delta \geqslant 7$. The proofs are mainly based on Theorems 16 and 18 .

Theorem 74 (Kang and Yuan [45], 2000). If $G$ is a connected planar graph, then $b(G) \leqslant \min \{8, \Delta(G)+2\}$.

Obviously, in view of Theorem 74, Conjecture 73 is true for any connected planar graph with $\Delta \geqslant 7$.

6.2. Bounds Implied by Degree Conditions. As we have seen from Theorem 74, to attack Conjecture 73, we only need to consider connected planar graphs with maximum $\Delta \leqslant 6$. Thus, studying the bondage number of planar graphs by degree conditions is of significance. The first result on bounds implied by degree conditions is obtained by Kang and Yuan [45]. 
Theorem 75 (Kang and Yuan [45], 2000). If G is a connected planar graph without vertices of degree 5 , then $b(G) \leqslant 7$. lows.

Fischermann et al. [46] generalized Theorem 75 as fol-

Theorem 76 (Fischermann et al. [46], 2003). Let G be a connected planar graph and $X$ the set of vertices of degree 5 which have distance at least 3 to vertices of degrees 1, 2, and 3. If all vertices in $X$ not adjacent with vertices of degree 4 are independent and not adjacent to vertices of degree 6 , then $b(G) \leqslant 7$.

Clearly, if $G$ has no vertices of degree 5 , then $X=\emptyset$. Thus, Theorem 76 yields Theorem 75 .

Use $n_{i}$ to denote the number of vertices of degree $i$ in $G$ for each $i=1,2, \ldots, \Delta(G)$. Using Theorem 18, Fischermann et al. obtained the following two theorems.

Theorem 77 (Fischermann et al. [46], 2003). For any connected planar graph $G$,

(1) $b(G) \leqslant 7$ if $n_{5}<2 n_{2}+3 n_{3}+2 n_{4}+12$;

(2) $b(G) \leqslant 6$ if $G$ contains no vertices of degrees 4 and 5 .

Theorem 78 (Fischermann et al. [46], 2003). For any connected planar graph $G, b(G) \leqslant \Delta(G)+1$ if

(1) $\Delta(G)=6$ and every edge $e=x y$ with $d_{G}(x)=5$ and $d_{G}(y)=6$ is contained in at most one triangle;

(2) $\Delta(G)=5$ and no triangle contains an edgee $=x y$ with $d_{G}(x)=5$ and $4 \leqslant d_{G}(y) \leqslant 5$.

6.3. Bounds Implied by Girth Conditions. The girth $g(G)$ of a graph $G$ is the length of the shortest cycle in $G$. If $G$ has no cycles, we define $g(G)=\infty$.

Combining Theorem 74 with Theorem 78, we find that if a planar graph contains no triangles and has maximum degree $\Delta \geqslant 5$, then Conjecture 73 holds. This fact motivated Fischermann et al's [46] attempt to attack Conjecture 73 by girth restraints.

Theorem 79 (Fischermann et al. [46], 2003). For any connected planar graph $G$,

$$
b(G) \leqslant \begin{cases}6 & \text { if } g(G) \geqslant 4, \\ 5 & \text { if } g(G) \geqslant 5, \\ 4 & \text { if } g(G) \geqslant 6, \\ 3 & \text { if } g(G) \geqslant 8 .\end{cases}
$$

The first result in Theorem 79 shows that Conjecture 73 is valid for all connected planar graphs with $g(G) \geqslant 4$ and $\Delta(G) \geqslant 5$. It is easy to verify that the second result in Theorem 79 implies that Conjecture 73 is valid for all not 3 -regular graphs of girth $g(G) \geqslant 5$, which is stated in the following corollary.

Corollary 80. For any connected planar graph $G$, if $G$ is not 3-regular and $g(G) \geqslant 5$, then $b(G) \leqslant \Delta(G)+1$.
The first result in Theorem 79 also implies that if $G$ is a connected planar graph with no cycles of length 3 , then $b(G) \leqslant 6$. Hou et al. [47] improved this result as follows.

Theorem 81 (Hou et al. [47], 2011). For any connected planar graph $G$, if $G$ contains no cycles of length $i(4 \leqslant i \leqslant 6)$, then $b(G) \leqslant 6$.

Since $b\left(C_{3 k+1}\right)=3$ for $k \geqslant 3$ (see Theorem 1), the last bound in Theorem 79 is tight. Whether other bounds in Theorem 79 are tight remains open. In 2003, Fischermann et al. [46] posed the following conjecture.

Conjecture 82. For any connected planar graph $G, b(G) \leqslant 7$, and

$$
b(G) \leqslant \begin{cases}5, & \text { if } g(G) \geqslant 4, \\ 4, & \text { if } g(G) \geqslant 5 .\end{cases}
$$

We conclude this subsection with a question on bondage numbers of planar graphs.

Question 1 (Fischermann et al. [46], 2003). Is there a planar graph $G$ with $6 \leqslant b(G) \leqslant 8$ ?

In 2006, Carlson and Develin [42] showed that the corona $G=H \circ K_{1}$ for a planar graph $H$ with $\delta(H)=5$ has the bondage number $b(G)=\delta(H)+1=6$ (see Theorem 66). Since the minimum degree of planar graphs is at most 5 , then $b(G)$ can attach 6 . If we take $H$ as the graph of the icosahedron, then $G=H \circ K_{1}$ is such an example. The question for the existence of planar graphs with bondage number 7 or 8 remains open.

6.4. Comments on the Conjectures. Conjecture 73 is true for all connected planar graphs with minimum degree $\delta \leqslant 2$ by Theorem 16, or maximum degree $\Delta \geqslant 7$ by Theorem 74 , or not $\gamma$-critical planar graphs by Theorem 13. Thus, to attack Conjecture 73, we only need to consider connected critical planar graphs with degree-restriction $3 \leqslant \delta \leqslant \Delta \leqslant 6$.

Recalling and anatomizing the proofs of all results mentioned in the preceding subsections on the bondage number for connected planar graphs, we find that the proofs of these results strongly depend upon Theorem 16 or Theorem 18. In other words, a basic way used in the proofs is to find two vertices $x$ and $y$ with distance at most two in a considered planar graph $G$ such that

$d_{G}(x)+d_{G}(y) \quad$ or $\quad d_{G}(x)+d_{G}(y)-\left|N_{G}(x) \cap N_{G}(y)\right|$,

which bounds $b(G)$, is as small as possible. Very recently, Huang and $\mathrm{Xu}[44]$ have considered the following parameter

$$
\begin{aligned}
B(G)=\min _{x, y \in V(G)}\left\{\left\{d_{x y}: 1 \leqslant d_{G}(x, y) \leqslant 2\right\}\right. \\
\left.\cup\left\{d_{x y}-n_{x y}: d(x, y)=1\right\}\right\},
\end{aligned}
$$

where $d_{x y}=d_{G}(x)+d_{G}(y)-1$ and $n_{x y}=\left|N_{G}(x) \cap N_{G}(y)\right|$. 
It follows from Theorems 16 and 18 that

$$
b(G) \leqslant B(G) .
$$

The proofs given in Theorems 74 and 79 indeed imply the following stronger results.

Theorem 83. If $G$ is a connected planar graph, then

$$
B(G) \leqslant \begin{cases}\min \{8, \Delta(G)+2\} \\ 6 \quad \text { if } g(G) \geqslant 4, \\ 5 \quad \text { if } g(G) \geqslant 5, \\ 4 \quad \text { if } g(G) \geqslant 6, \\ 3 \quad \text { if } g(G) \geqslant 8 .\end{cases}
$$

Thus, using Theorem 16 or Theorem 18, if we can prove Conjectures 73 and 82, then we can prove the following statement.

Statement. If $G$ is a connected planar graph, then

$$
B(G) \leqslant \begin{cases}\Delta(G)+1, & \\ 7, & \text { if } g(G) \geqslant 4, \\ 5, & \text { if } g(G) \geqslant 5 .\end{cases}
$$

It follows from (24) that Statement implies Conjectures 73 and 82. However, Huang and $\mathrm{Xu}[44]$ gave examples to show that none of conclusions in Statement is true. As a result, they stated the following conclusion.

Theorem 84 (Huang and $\mathrm{Xu}$ [44], 2012). It is not possible to prove Conjectures 73 and 82, if they are right, using Theorems 16 and 18.

Therefore, a new method is needed to prove or disprove these conjectures. At the present time, one cannot prove these conjectures, and may consider to disprove them. If one of these conjectures is invalid, then there exists a minimum counterexample $G$ with respect to $v(G)+\varepsilon(G)$. In order to obtain a minimum counterexample, one may consider two simple operations satisfying these requirements, the edgedeletion and the edge-contraction, which decrease $v(G)+\mathcal{E}(G)$ and preserve planarity. However, applying Theorems 71 and 72, Huang and $\mathrm{Xu}[44]$ presented the following results.

Theorem 85 (Huang and $\mathrm{Xu}$ [44], 2012). It is not possible to construct minimum counterexamples to Conjectures 73 and 82 by the operation of an edge-deletion or an edge-contraction.

\section{Results on Crossing Number Restraints}

It is quite natural to generalize the known results on the bondage number for planar graphs to for more general graphs in terms of graph-theoretical parameters. In this section, we consider graphs with crossing number restraints.

The crossing number $\operatorname{cr}(G)$ of $G$ is the smallest number of pairwise intersections of its edges when $G$ is drawn in the plane. If $\operatorname{cr}(G)=0$, then $G$ is a planar graph.
7.1. General Methods. Use $n_{i}$ to denote the number of vertices of degree $i$ in $G$ for $i=1,2, \ldots, \Delta(G)$. Huang and $\mathrm{Xu}$ obtained the following results.

Theorem 86 (Huang and $\mathrm{Xu}$ [48], 2007). For any connected graph $G$,

$$
b(G) \leqslant \begin{cases}6 \quad \text { if } g(G) \geqslant 4 \text { and } 2 \operatorname{cr}(G)<\frac{1}{2} a_{1} ; \\ 5 \quad \text { if } g(G) \geqslant 5 \text { and } 6 \operatorname{cr}(G)<\frac{1}{6} a_{2} ; \\ 4 \quad \text { if } g(G) \geqslant 6 \text { and } 4 \operatorname{cr}(G)<\frac{1}{4} a_{3} ; \\ 3 \quad \text { if } g(G) \geqslant 8 \text { and } 6 \operatorname{cr}(G)<\frac{1}{6} a_{4},\end{cases}
$$

where $a_{1}=n_{1}+2 n_{2}+2 n_{3}+\sum_{i=8}^{\Delta}(i-7) n_{i}+8 ; a_{2}=3 n_{1}+6 n_{2}+$ $5 n_{3}+\sum_{i=7}^{\Delta}(3 i-18) n_{i}+20 ; a_{3}=n_{1}+2 n_{2}+\sum_{i=6}^{\Delta}(2 i-10) n_{i}+12$; and $a_{4}=\sum_{i=5}^{\Delta}(3 i-12) n_{i}+16$.

Simplifying the conditions in Theorem 86 , we obtain the following corollaries immediately.

Corollary 87 (Huang and $\mathrm{Xu}[48], 2007)$. For any connected graph $G$,

$$
b(G) \leqslant \begin{cases}6, & \text { if } g(G) \geqslant 4 \text { and } \operatorname{cr}(G) \leqslant 3, \\ 5, & \text { if } g(G) \geqslant 5 \text { and } \operatorname{cr}(G) \leqslant 4, \\ 4, & \text { if } g(G) \geqslant 6 \text { and } \operatorname{cr}(G) \leqslant 2, \\ 3, & \text { if } g(G) \geqslant 8 \text { and } \operatorname{cr}(G) \leqslant 2 .\end{cases}
$$

Corollary 88 (Huang and $\mathrm{Xu}$ [48], 2007). For any connected graph $G$,

(a) $b(G) \leqslant 6$ if $G$ is not 4-regular, $\operatorname{cr}(G)=4$ and $g(G) \geqslant 4$;

(b) $b(G) \leqslant \Delta(G)+1$ if $G$ is not 3-regular, $\operatorname{cr}(G) \leqslant 4$ and $g(G) \geqslant 5$

(c) $b(G) \leqslant 4$ if $G$ is not 3-regular, $\operatorname{cr}(G)=3$ and $g(G) \geqslant 6$;

(d) $b(G) \leqslant 3$ if $\operatorname{cr}(G)=3, g(G) \geqslant 8$ and $\Delta(G) \geqslant 5$.

These corollaries generalize some known results for planar graphs. For example, Corollary 87 contains Theorem 79; Corollary 88 (b) contains Corollary 80.

Theorem 89 (Huang and $\mathrm{Xu}[48], 2007)$. For any connected graph $G$, if $\operatorname{cr}(G) \leqslant n_{3}(G)+n_{4}(G)+3$, then $b(G) \leqslant 8$.

Corollary 90 (Huang and $\mathrm{Xu}$ [48], 2007). For any connected graph $G$, if $\operatorname{cr}(G) \leqslant 3$, then $b(G) \leqslant 8$.

Perhaps being unaware of this result, in 2010, Ma et al. [49] proved that $b(G) \leqslant 12$ for any graph $G$ with $\operatorname{cr}(G)=1$.

Theorem 91 (Huang and $\mathrm{Xu}[48], 2007)$. Let $\mathrm{G}$ be a connected graph, and $I=\left\{x \in V(G): d_{G}(x)=5, d_{G}(x, y) \geqslant 3\right.$ if 
$d_{G}(y) \leqslant 3$, and $d_{G}(y) \neq 4$ for every $\left.y \in N_{G}(x)\right\}$. If $I$ is independent, no vertices adjacent to vertices of degree 6 and

$$
\operatorname{cr}(G)<\max \left\{\frac{5 n_{3}+|I|-2 n_{4}+28}{11}, \frac{7 n_{3}+40}{16}\right\},
$$

then $b(G) \leqslant 7$.

Corollary 92 (Huang and $\mathrm{Xu}$ [48], 2007). Let $G$ be a connected graph with $\mathrm{cr}(G) \leqslant 2$. If $I=\left\{x \in V(G): d_{G}(x)=\right.$ $5, d_{G}(y, x) \geqslant 3$ if $d_{G}(y) \leqslant 3$ and $d_{G}(y) \neq 4$ for every $y \in$ $\left.N_{G}(x)\right\}$ is independent, and no vertices adjacent to vertices of degree 6 , then $b(G) \leqslant 7$.

Theorem 93 (Huang and $\mathrm{Xu}$ [48], 2007). Let $\mathrm{G}$ be a connected graph. If $G$ satisfies
(a) $5 \mathrm{cr}(G)+n_{5}<2 n_{2}+3 n_{3}+2 n_{4}+12$ or
(b) $7 \mathrm{cr}(G)+2 n_{5}<3 n_{2}+4 n_{4}+24$,

then $b(G) \leqslant 7$.

Proposition 94 (Huang and $\mathrm{Xu}$ [48], 2007). Let $G$ be a connected graph with no vertices of degrees four and five. If $\operatorname{cr}(G) \leqslant 2$, then $b(G) \leqslant 6$.

Theorem 95 (Huang and $\mathrm{Xu}$ [48], 2007). If $\mathrm{G}$ is a connected graph with $\operatorname{cr}(G) \leqslant 4$ and not 4-regular when $\operatorname{cr}(G)=4$, then $b(G) \leqslant \Delta(G)+2$.

The above results generalize some known results for planar graphs. For example, Corollary 90 and Theorem 95 contain Theorem 74; the first condition in Theorem 93 contains the second condition in Theorem 77.

From Corollary 88 and Theorem 95, we suggest the following questions.

Question 2. Is there a

(a) 4-regular graph $G$ with $\operatorname{cr}(G)=4$ such that $b(G) \geqslant 7$ ?

(b) 3-regular graph $G$ with $\mathrm{cr} \leqslant 4$ and $g(G) \geqslant 5$ such that $b(G)=5$ ?

(c) 3-regular graph $G$ with $\mathrm{cr}=3$ and $g(G)=6$ or 7 such that $b(G)=5$ ?

7.2. Carlson-Develin Methods. In this subsection, we introduce an elegant method presented by Carlson and Develin [42] to obtain some upper bounds for the bondage number of a graph.

Suppose that $G$ is a connected graph. We say that $G$ has genus $\rho$ if $G$ can be embedded in a surface $S$ with $\rho$ handles such that edges are pairwise disjoint except possibly for endvertices. Let $\widetilde{G}$ be an embedding of $G$ in surface $S$, and let $\phi(G)$ denote the number of regions in $\widetilde{G}$. The boundary of every region contains at least three edges and every edge is on the boundary of at most two regions (the two regions are identical when $e$ is a cut-edge). For any edge $e$ of $G$, let $r_{G}^{1}(e)$ and $r_{G}^{2}(e)$ be the numbers of edges comprising the regions in $\widetilde{G}$ which the edge $e$ borders. It is clear that every $e=x y \in E(G)$,

$$
\begin{gathered}
r_{G}^{2}(e) \geqslant r_{G}^{1}(e) \geqslant 4 \quad \text { if }\left|N_{G}(x) \cap N_{G}(y)\right|=0, \\
r_{G}^{2}(e) \geqslant 4, \quad r_{G}^{1}(e) \geqslant 3 \text { if }\left|N_{G}(x) \cap N_{G}(y)\right|=1, \\
r_{G}^{2}(e) \geqslant r_{G}^{1}(e) \geqslant 3 \quad \text { if }\left|N_{G}(x) \cap N_{G}(y)\right| \geqslant 2 .
\end{gathered}
$$

Following Carlson and Develin [42], for any edge $e=x y$ of $G$, we define

$$
D_{G}(e)=\frac{1}{d_{G}(x)}+\frac{1}{d_{G}(y)}+\frac{1}{r_{G}^{1}(e)}+\frac{1}{r_{G}^{2}(e)}-1 .
$$

By the well-known Euler's formula

$$
v(G)-\varepsilon(G)+\phi(G)=2-2 \rho(G),
$$

it is easy to see that

$$
\sum_{e \in E(G)} D_{G}(e)=v(G)-\varepsilon(G)+\phi(G)=2-2 \rho(G) .
$$

If $G$ is a planar graph, that is, $\rho(G)=0$, then

$$
\sum_{e \in E(G)} D_{G}(e)=v(G)-\varepsilon(G)+\phi(G)=2 .
$$

Combining these formulas with Theorem 18, Carlson and Develin [42] gave a simple and intuitive proof of Theorem 74 and obtained the following result.

Theorem 96 (Carlson and Develin [42], 2006). Let $G$ be a connected graph which can be embedded on a torus. Then $b(G) \leqslant \Delta(G)+3$.

Recently, Hou and Liu [50] improved Theorem 96 as follows.

Theorem 97 (Hou and Liu [50], 2012). Let $G$ be a connected graph which can be embedded on a torus. Then $b(G) \leqslant 9$. Moreover, if $\Delta(G) \neq 6$, then $b(G) \leqslant 8$.

By the way, Cao et al. [51] generalized the result in Theorem 79 to a connected graph $G$ that can be embedded on a torus; that is,

$$
b(G) \leq \begin{cases}6, & \text { if } g(G) \geqslant 4 \text { and } G \text { is not 4-regular, } \\ 5, & \text { if } g(G) \geqslant 5 \\ 4, & \text { if } g(G) \geqslant 6 \text { and } G \text { is not 3-regular, } \\ 3, & \text { if } g(G) \geqslant 8\end{cases}
$$

Several authors used this method to obtain some results on the bondage number. For example, Fischermann et al. [46] used this method to prove the second conclusion in Theorem 78. Recently, Cao et al. [52] have used this method to deal with more general graphs with small crossing numbers. First, they found the following property.

Lemma 98. $\delta(G) \leqslant 5$ for any graph $G$ with $\operatorname{cr}(G) \leqslant 5$. 
This result implies that $b(G) \leqslant \Delta(G)+4$ by Corollary 15 for any graph $G$ with $\operatorname{cr}(G) \leqslant 5$. Cao et al. established the following relation in terms of maximum planar subgraphs.

Lemma 99 (Cao et al. [52]). Let $G$ be a connected graph with crossing number $\operatorname{cr}(G)$. For any maximum planar subgraph $H$ of $G$,

$$
\sum_{e \in E(H)} D_{G}(e) \geqslant 2-\frac{2 \operatorname{cr}(G)}{\delta(G)} .
$$

Using Carlson-Develin method and combining Lemma 98 with Lemma 99, Cao et al. proved the following results.

Theorem 100 (Cao et al. [52]). For any connected graph $G$, $b(G) \leqslant \Delta(G)+2$ if $G$ satisfies one of the following conditions:

(a) $\operatorname{cr}(G) \leqslant 3$;

(b) $\operatorname{cr}(G)=4$ and $G$ is not 4-regular;

(c) $\operatorname{cr}(G)=5$ and $G$ contains no vertices of degree 4 .

Theorem 101 (Cao et al. [52]). Let $G$ be a connected graph with $\Delta(G)=5$ and $\operatorname{cr}(G) \leqslant 4$. If no triangles contain two vertices of degree 5 , then $b(G) \leqslant 6=\Delta(G)+1$.

Theorem 102 (Cao et al. [52]). Let $G$ be a connected graph with $\Delta(G) \geqslant 6$ and $\operatorname{cr}(G) \leqslant 3$. If $\Delta(G) \geqslant 7$ or if $\Delta(G)=6$, $\delta(G) \neq 3$ and every edge $e=x y$ with $d_{G}(x)=5$ and $d_{G}(y)=6$ is contained in at most one triangle, then $b(G) \leqslant \min \{8, \Delta(G)+$ $1\}$.

Using Carlson-Develin method, it can be proved that $b(G) \leqslant \Delta(G)+2$ if $\operatorname{cr}(G) \leqslant 3$ (see the first conclusion in Theorem 100), and not yet proved that $b(G) \leqslant 8$ if $\operatorname{cr}(G) \leqslant$ 3 , although it has been proved by using other method (see Corollary 90).

By using Carlson-Develin method, Samodivkin [25] obtained some results on the bondage number for graphs with some given properties.

Kim [53] showed that $b(G) \leqslant \Delta(G)+2$ for a connected graph $G$ with genus $1, \Delta(G) \leqslant 5$ and having a toroidal embedding of which at least one region is not 4 -sided. Recently, Gagarin and Zverovich [54] further have extended Carlson-Develin ideas to establish a nice upper bound for arbitrary graphs that can be embedded on orientable or nonorientable topological surface.

Theorem 103 (Gagarin and Zverovich [54], 2012). Let $G$ be a graph embeddable on an orientable surface of genus $h$ and $a$ nonorientable surface of genus $k$. Then $b(G) \leqslant \min \{\Delta(G)+h+$ $2, \Delta(G)+k+1\}$.

This result generalizes the corresponding upper bounds in Theorems 74 and 96 for any orientable or nonorientable topological surface. By investigating the proof of Theorem 103, Huang [55] found that the issue of the orientability can be avoided by using the Euler characteristic $\chi(=v(G)-\varepsilon(G)+\phi(G))$ instead of the genera $h$ and $k$; the relations are $\chi=2-2 h$ and $\chi=2-k$. To obtain the best result from Theorem 103, one wants $h$ and $k$ as small as possible; this is equivalent to having $\chi$ as large as possible.

According to Theorem 103, if $G$ is planar $(h=0, \chi=2)$ or can be embedded on the real projective plane $(k=1, \chi=1)$, then $b(G) \leqslant \Delta(G)+2$. In all other cases, Huang [55] had the following improvement for Theorem 103; the proof is based on the technique developed by Carlson-Develin and GagarinZverovich and includes some elementary calculus as a new ingredient, mainly the intermediate-value theorem and the mean-value theorem.

Theorem 104 (Huang [55], 2012). Let $G$ be a graph embeddable on a surface whose Euler characteristic $\chi$ is as large as possible. If $\chi \leqslant 0$, then $b(G) \leqslant \Delta(G)+\lfloor r\rfloor$, where $r$ is the largest real root of the following cubic equation in $z$ :

$$
z^{3}+2 z^{2}+(6 \chi-7) z+18 \chi-24=0
$$

In addition, if $\chi$ decreases, then $r$ increases.

The following result is asymptotically equivalent to Theorem 104.

Theorem 105 (Huang [55], 2012). Let $G$ be a graph embeddable on a surface whose Euler characteristic $\chi$ is as large as possible. If $\chi \leqslant 0$, then $b(G)<\Delta(G)+\sqrt{12-6 \chi}+1 / 2$, or equivalently, $b(G) \leqslant \Delta(G)+\lceil\sqrt{12-6 \chi}-1 / 2\rceil$.

Also, Gagarin and Zverovich [54] indicated that the upper bound in Theorem 103 can be improved for larger values of the genera $h$ and $k$ by adjusting the proofs and stated the following general conjecture.

Conjecture 106 (Gagarin and Zverovich [54], 2012). For a connected graph $G$ of orientable genus $h$ and nonorientable genus $k$,

$$
b(G) \leqslant \min \left\{c_{h}, c_{k}^{\prime}, \Delta(G)+o(h), \Delta(G)+o(k)\right\},
$$

where $c_{h}$ and $c_{k}^{\prime}$ are constants depending, respectively, on the orientable and nonorientable genera of $G$.

In the recent paper, Gagarin and Zverovich [56] provided constant upper bounds for the bondage number of graphs on topological surfaces, which can be used as the first estimation for the constants $c_{h}$ and $c_{k}^{\prime}$ of Conjecture 106.

Theorem 107 (Gagarin and Zverovich [56], 2013). Let $G$ be a connected graph of order $n$. If $G$ can be embedded on the surface of its orientable or nonorientable genus of the Euler characteristic $\chi$, then

(a) $\chi \geqslant 1$ implies $b(G) \leqslant 10$

(b) $\chi \leqslant 0$ and $n>-12 \chi$ imply $b(G) \leqslant 11$;

(c) $\chi \leqslant-1$ and $n \leqslant-12 \chi$ imply $b(G) \leqslant 11+$ $3 \chi(\sqrt{17-8 \chi}-3) /(\chi-1)=11+O(\sqrt{-\chi})$.

Theorem 79 shows that a connected planar triangle-free graph $G$ has $b(G) \leqslant 6$. The following result generalizes to it all the other topological surfaces. 
Theorem 108 (Gagarin and Zverovich [56], 2013). Let G be a connected triangle-free graph of order $n$. If $G$ can be embedded on the surface of its orientable or nonorientable genus of the Euler characteristic $\chi$, then

(a) $\chi \geqslant 1$ implies $b(G) \leqslant 6$;

(b) $\chi \leqslant 0$ and $n>-8 \chi$ imply $b(G) \leqslant 7$;

(c) $\chi \leqslant-1$ and $n \leqslant-8 \chi$ imply $b(G) \leqslant 7-4 \chi /(1+\sqrt{2-\chi})$.

In [56], the authors also explicitly improved upper bounds in Theorem 103 and gave tight lower bounds for the number of vertices of graphs 2-cell embeddable on topological surfaces of a given genus. The interested reader is referred to the original paper for details. The following result is interesting although its proof is easy.

Theorem 109 (Samodivkin [57]). Let $G$ be a graph with order $n$ and girth $g$. If $G$ is embeddable on a surface whose Euler characteristic $\chi$ is as large as possible, then

$$
b(G) \leqslant 3+\frac{8}{g-2}-\frac{4 \chi g}{n(g-2)} .
$$

If $G$ is a planar graph with girth $g \geqslant 4+i$ for any $i \epsilon$ $\{0,1,2\}$, then Theorem 109 leads to $b(G) \leqslant 6-i$, which is the result in Theorem 79 obtained by Fischermann et al. [46]. Also, in many cases the bound stated in Theorem 109 is better than those given by Theorems 103 and 105.

\section{Conditional Bondage Numbers}

Since the concept of the bondage number is based upon the domination, all sorts of dominations, which are variations of the normal domination by adding restricted conditions to the normal dominating set, can develop a new "bondage number" as long as a variation of domination number is given. In this section, we survey results on the bondage number under some restricted conditions.

8.1. Total Bondage Numbers. A dominating set $S$ of a graph $G$ without isolated vertices is called total if the subgraph induced by $S$ contains no isolated vertices. The minimum cardinality of a total dominating set is called the total domination number of $G$ and denoted by $\gamma_{T}(G)$. It is clear that $\gamma(G) \leqslant \gamma_{T}(G) \leqslant 2 \gamma(G)$ for any graph $G$ without isolated vertices.

The total domination in graphs was introduced by Cockayne et al. [58] in 1980. Pfaff et al. [59, 60] in 1983 showed that the problem determining total domination number for general graphs is NP-complete, even for bipartite graphs and chordal graphs. Even now, total domination in graphs has been extensively studied in the literature. In 2009, Henning [61] gave a survey of selected recent results on total domination in graphs.

The total bondage number of $G$, denoted by $b_{T}(G)$, is the smallest cardinality of a subset $B \subseteq E(G)$ with the property that $G-B$ contains no isolated vertices and $\gamma_{T}(G-B)>\gamma_{T}(G)$.

From definition, $b_{T}(G)$ may not exist for some graphs, for example, $G=K_{1, n}$. We put $b_{T}(G)=\infty$ if $b_{T}(G)$ does not exist.
It is easy to see that $b_{T}(G)$ is finite for any connected graph $G$ other than $K_{1}, K_{2}, K_{3}$, and $K_{1, n}$. In fact, for such a graph $G$, since there is a path of length 3 in $G$, we can find $B_{1} \subseteq E(G)$ such that $G-B_{1}$ is a spanning tree $T$ of $G$, containing a path of length 3. So $\gamma_{T}(T)=\gamma_{T}\left(G-B_{1}\right) \geqslant \gamma_{T}(G)$. For the tree $T$, we find $B_{2} \subseteq E(T)$ such that $\gamma_{T}\left(T-B_{2}\right)>\gamma_{T}(T) \geqslant \gamma_{T}(G)$. Thus, we have $\gamma_{T}\left(G-B_{2}-B_{1}\right)>\gamma_{T}(G)$, and so $b_{T}(G) \leqslant\left|B_{1}\right|+\left|B_{2}\right|$. In the following discussion, we always assume that $b_{T}(G)$ exists when a graph $G$ is mentioned.

In 1991, Kulli and Patwari [62] first studied the total bondage number of a graph and calculated the exact values of $b_{T}(G)$ for some standard graphs.

Theorem 110 (Kulli and Patwari [62], 1991). For $n \geqslant 4$,

$$
\begin{aligned}
& b_{T}\left(C_{n}\right)= \begin{cases}3 & \text { if } n \equiv 2(\bmod 4), \\
2 & \text { otherwise, }\end{cases} \\
& b_{T}\left(P_{n}\right)= \begin{cases}2 & \text { if } n \equiv 2(\bmod 4), \\
1 & \text { otherwise, }\end{cases} \\
& b_{T}\left(K_{m, n}\right)=m \text { with } 2 \leqslant m \leqslant n, \\
& b_{T}\left(K_{n}\right)= \begin{cases}4 & \text { for } n=4, \\
2 n-5 & \text { for } n \geqslant 5 .\end{cases}
\end{aligned}
$$

Recently, Hu et al. [63] have obtained some results on the total bondage number of the Cartesian product $P_{m} \times P_{n}$ of two paths $P_{m}$ and $P_{n}$.

Theorem 111 (Hu et al. [63], 2012). For the Cartesian product $P_{m} \times P_{n}$ of two paths $P_{m}$ and $P_{n}$,

$$
\begin{gathered}
b_{T}\left(P_{2} \times P_{n}\right)= \begin{cases}1 & \text { if } n \equiv 0(\bmod 3), \\
2 & \text { if } n \equiv 2(\bmod 3), \\
3 & \text { if } n \equiv 1(\bmod 3),\end{cases} \\
b_{T}\left(P_{4} \times P_{n}\right) \begin{cases}=1 & \text { if } n \equiv 1(\bmod 5), \\
=2 & \text { if } n \equiv 4(\bmod 5), \\
\leqslant 3 & \text { if } n \equiv 2(\bmod 5), \\
\leqslant 4 & \text { if } n \equiv 0,3(\bmod 5) .\end{cases}
\end{gathered}
$$

Generalized Petersen graphs are an important class of commonly used interconnection networks and have been studied recently. By constructing a family of minimum total dominating sets, Cao et al. [64] determined the total bondage number of the generalized Petersen graphs.

From Theorem 6 , we know that $b(T) \leqslant 2$ for a nontrivial tree $T$. But given any positive integer $k$, Sridharan et al. [65] constructed a tree $T$ for which $b_{T}(T)=k$. Let $H_{k}$ be the tree obtained from a star $K_{1, k+1}$ by subdividing $k$ edges twice. The tree $\mathrm{H}_{7}$ is shown in Figure 6. It can be easily verified that $b_{T}\left(H_{k}\right)=k$. This fact can be stated as the following theorem.

Theorem 112 (Sridharan et al. [65], 2007). For any positive integer $k$, there exists a tree $T$ with $b_{T}(T)=k$. 


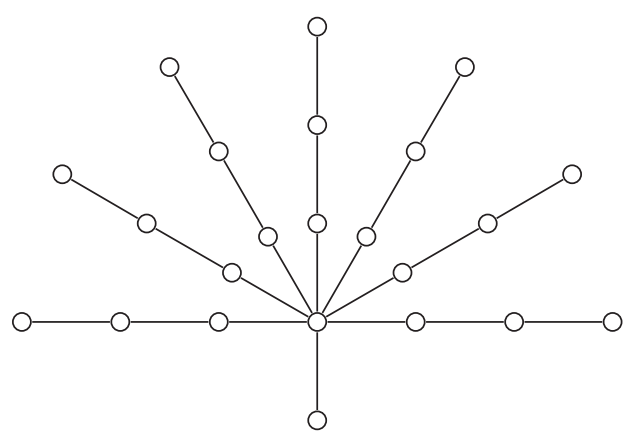

FIGURE 6: $\mathrm{H}_{7}$. follows

Combining Theorem 1 with Theorem 112, we have what

Corollary 113 (Sridharan et al. [65], 2007). The bondage number and the total bondage number are unrelated, even for trees.

However, Sridharan et al. [65] gave an upper bound on the total bondage number of a tree in terms of its maximum degree. For any tree $T$ of order $n$, if $T \neq K_{1, n-1}$, then $b_{T}(T)=$ $\min \{\Delta(T),(1 / 3)(n-1)\}$. Rad and Raczek [66] improved this upper bound and gave a constructive characterization of a certain class of trees attaching the upper bound.

Theorem 114 (Rad and Raczek [66], 2011). $b_{T}(T) \leqslant \Delta(T)-1$ for any tree $T$ with maximum degree at least three.

In general, the decision problem for $b_{T}(G)$ is NP-complete for any graph $G$. We state the decision problem for the total bondage as follows.

Problem 3. Consider the decision problem:

Total Bondage Problem

Instance: a graph $G$ and a positive integer $b$.

Question: is $b_{T}(G) \leqslant b$ ?

Theorem 115 ( $\mathrm{Hu}$ and $\mathrm{Xu}[16], 2012)$. The total bondage problem is NP-complete.

Consequently, in view of its computational hardness, it is significative to establish some sharp bounds on the total bondage number of a graph in terms of other graphic parameters. In 1991, Kulli and Patwari [62] showed that $b_{T}(G) \leqslant 2 n-5$ for any graph $G$ with order $n \geqslant 5$. Sridharan et al. [65] improved this result as follows.

Theorem 116 (Sridharan et al. [65], 2007). For any graph $G$ with order $n$, if $n \geqslant 5$, then

$$
b_{T}(G) \leqslant \begin{cases}\frac{1}{3}(n-1) & \text { if } G \text { contains no cycles, } \\ n-1 & \text { if } g(G) \geqslant 5 \\ n-2 & \text { if } g(G)=4\end{cases}
$$

Rad and Raczek [66] also established some upper bounds on $b_{T}(G)$ for a general graph $G$. In particular, they gave an upper bound on $b_{T}(G)$ in terms of the girth of G.

Theorem 117 (Rad and Raczek [66], 2011). $b_{T}(G) \leqslant 4 \Delta(G)-5$ for any graph $G$ with $g(G) \geqslant 4$.

8.2. Paired Bondage Numbers. A dominating set $S$ of $G$ is called to be paired if the subgraph induced by $S$ contains a perfect matching. The paired domination number of $G$, denoted by $\gamma_{P}(G)$, is the minimum cardinality of a paired dominating set of $G$. Clearly, $\gamma_{T}(G) \leqslant \gamma_{P}(G)$ for every connected graph $G$ with order at least two, where $\gamma_{T}(G)$ is the total domination number of $G$, and $\gamma_{P}(G) \leqslant 2 \gamma(G)$ for any graph $G$ without isolated vertices. Paired domination was introduced by Haynes and Slater $[67,68]$ and further studied in [69-72].

The paired bondage number of $G$ with $\delta(G) \geqslant 1$, denoted by $b_{\mathrm{P}}(G)$, is the minimum cardinality among all sets of edges $B \subseteq E$ such that $\delta(G-B) \geqslant 1$ and $\gamma_{P}(G-B)>\gamma_{P}(G)$.

The concept of the paired bondage number was first proposed by Raczek [73] in 2008. The following observations follow immediately from the definition of the paired bondage number.

Observation 2. Let $G$ be a graph with $\delta(G) \geqslant 1$.

(a) If $H$ is a subgraph of $G$ such that $\delta(H) \geqslant 1$, then $b_{P}(H) \leqslant b_{P}(G)$.

(b) If $H$ is a subgraph of $G$ such that $b_{P}(H)=1$ and $k$ is the number of edges removed to form $H$, then $1 \leqslant$ $b_{P}(G) \leqslant k+1$.

(c) If $x y \in E(G)$ such that $d_{G}(x), d_{G}(y)>1$, and $x y$ belongs to each perfect matching of each minimum paired dominating set of $G$, then $b_{P}(G)=1$.

Based on these observations, $b_{P}\left(P_{n}\right) \leqslant 2$. In fact, the paired bondage number of a path has been determined.

Theorem 118 (Raczek [73], 2008). For any positive integer $k$, if $n \geqslant 2$, then

$$
b_{P}\left(P_{n}\right)= \begin{cases}0 & \text { if } n=2,3, \text { or } 5, \\ 1 & \text { if } n=4 k, 4 k+3, \text { or } 4 k+6, \\ 2 & \text { otherwise. }\end{cases}
$$

For a cycle $C_{n}$ of order $n \geqslant 3$, since $\gamma_{P}\left(C_{n}\right)=\gamma_{P}\left(P_{n}\right)$, from Theorem 118 the following result holds immediately.

Corollary 119 (Raczek [73], 2008). For any positive integer $k$, if $n \geqslant 3$, then

$$
b_{P}\left(C_{n}\right)= \begin{cases}0 & \text { if } n=3 \text { or } 5 \\ 2 & \text { if } n=4 k, 4 k+3, \text { or } 4 k+6 \\ 3 & \text { otherwise. }\end{cases}
$$

A wheel $W_{n}$, where $n \geqslant 4$, is a graph with $n$ vertices, formed by connecting a single vertex to all vertices of a cycle $C_{n-1}$. Of course $\gamma_{P}\left(W_{n}\right)=2$. 


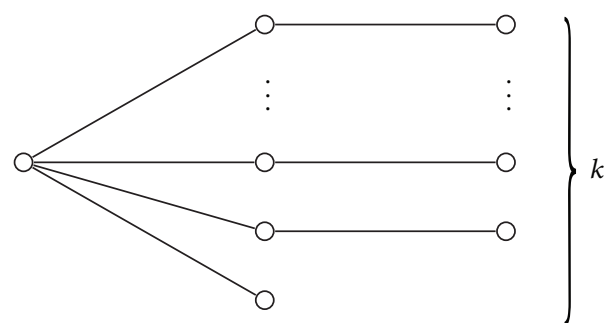

Figure 7: A tree $T$ with $b_{P}(T)=k$.

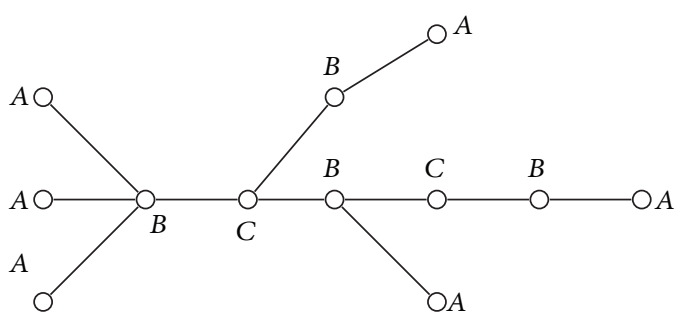

FIgURE 8: A tree $T$ belong to the family $\mathscr{T}$.
Theorem 120 (Raczek [73], 2008). For positive integers $n$ and $m$,

$$
\begin{gathered}
b_{P}\left(K_{m, n}\right)=m \quad \text { for } 1<m \leqslant n, \\
b_{P}\left(W_{n}\right)= \begin{cases}4 & \text { if } n=4, \\
3 & \text { if } n=5, \\
2 & \text { otherwise. }\end{cases}
\end{gathered}
$$

Theorem 16 shows that if $T$ is a nontrivial tree, then $b(T) \leqslant 2$. However, no similar result exists for paired bondage. For any nonnegative integer $k$, let $T_{k}$ be a tree obtained by subdividing all but one edge of a star $K_{1, k+1}$ (as shown in Figure 7). It is easy to see that $b_{P}\left(T_{k}\right)=k$. We state this fact as the following theorem, which is similar to Theorem 112.

Theorem 121 (Raczek [73], 2008). For any nonnegative integer $k$, there exists a tree $T$ with $b_{P}(T)=k$.

Consider the tree defined in Figure 5 for $k=3$. Then $b\left(T_{3}\right) \leqslant 2$ and $b_{P}\left(T_{3}\right)=3$. On the other hand, $b\left(P_{4}\right)=2$ and $b_{P}\left(P_{4}\right)=1$. Thus, we have what follows, which is similar to Corollary 113.

Corollary 122. The bondage number and the paired bondage number are unrelated, even for trees.

A constructive characterization of trees with $b(T)=2$ is given by Hartnell and Rall in [12]. Raczek [73] provided a constructive characterization of trees with $b_{P}(T)=0$. In order to state the characterization, we define a labeling and three simple operations on a tree $T$. Let $y \in V(T)$ and let $\ell(y)$ be the label assigned to $y$.

Operation $\mathscr{T}_{1}$. If $\ell(y)=B$, add a vertex $x$ and the edge $y x$, and let $\ell(x)=A$.

Operation $\mathscr{T}_{2}$. If $\ell(y)=C$, add a path $\left(x_{1}, x_{2}\right)$ and the edge $y x_{1}$, and let $\ell\left(x_{1}\right)=B, \ell\left(x_{2}\right)=A$.

Operation $\mathscr{T}_{3}$. If $\ell(y)=B$, add a path $\left(x_{1}, x_{2}, x_{3}\right)$ and the edge $y x_{1}$, and let $\ell\left(x_{1}\right)=C, \ell\left(x_{2}\right)=B$, and $\ell\left(x_{3}\right)=A$.

Let $P_{2}=(u, v)$ with $\ell(u)=A$ and $\ell(v)=B$. Let $\mathscr{T}$ be the class of all trees obtained from the labeled $P_{2}$ by a finite sequence of operations $\mathscr{T}_{1}, \mathscr{T}_{2}, \mathscr{T}_{3}$.

A tree $T$ in Figure 8 belongs to the family $\mathscr{T}$.

Raczek [73] obtained the following characterization of all trees $T$ with $b_{P}(T)=0$.
Theorem 123 (Raczek [73], 2008). For a tree $T, b_{P}(T)=0$ if and only if $T$ is in $\mathscr{T}$. follows.

We state the decision problem for the paired bondage as

Problem 4. Consider the decision problem:

Paired Bondage Problem

Instance: a graph $G$ and a positive integer $b$.

Question: is $b_{P}(G) \leqslant b$ ?

Conjecture 124. The paired bondage problem is NP-complete.

8.3. Restrained Bondage Numbers. A dominating set $S$ of $G$ is called to be restrained if the subgraph induced by $\bar{S}$ contains no isolated vertices, where $\bar{S}=V(G) \backslash S$. The restrained domination number of $G$, denoted by $\gamma_{R}(G)$, is the smallest cardinality of a restrained dominating set of $G$. It is clear that $\gamma_{R}(G)$ exists and $\gamma(G) \leqslant \gamma_{R}(G)$ for any nonempty graph $G$.

The concept of restrained domination was introduced by Telle and Proskurowski [74] in 1997, albeit indirectly, as a vertex partitioning problem. Concerning the study of the restrained domination numbers, the reader is referred to [7479].

In 2008, Hattingh and Plummer [80] defined the restrained bondage number $b_{\mathrm{R}}(G)$ of a nonempty graph $G$ to be the minimum cardinality among all subsets $B \subseteq E(G)$ for which $\gamma_{R}(G-B)>\gamma_{R}(G)$.

For some simple graphs, their restrained domination numbers can be easily determined, and so restrained bondage numbers have been also determined. For example, it is clear that $\gamma_{R}\left(K_{n}\right)=1$. Domke et al. [77] showed that $\gamma_{R}\left(C_{n}\right)=$ $n-2\lfloor n / 3\rfloor$ for $n \geqslant 3$, and $\gamma_{R}\left(P_{n}\right)=n-2\lfloor(n-1) / 3\rfloor$ for $n \geqslant 1$. Using these results, Hattingh and Plummer [80] obtained the restricted bondage numbers for $K_{n}, C_{n}, P_{n}$, and $G=K_{n_{1}, n_{2}, \ldots, n_{t}}$ as follows.

Theorem 125 (Hattingh and Plummer [80], 2008). For $n \geqslant 3$,

$$
\begin{gathered}
b_{R}\left(K_{n}\right)= \begin{cases}1 & \text { if } n=3, \\
\left\lceil\frac{n}{2}\right] & \text { otherwise, }\end{cases} \\
b_{R}\left(C_{n}\right)= \begin{cases}1 & \text { if } n \equiv 0(\bmod 3), \\
2 & \text { otherwise, }\end{cases} \\
b_{R}\left(P_{n}\right)=1, \quad n \geqslant 4,
\end{gathered}
$$


$b_{R}(G)$

$$
= \begin{cases}\left\lceil\frac{m}{2}\right\rceil & \text { if } n_{m}=1 \text { and } n_{m+1} \geqslant 2(1 \leqslant m<t), \\ 2 t-2 & \text { if } n_{1}=n_{2}=\cdots=n_{t}=2(t \geqslant 2), \\ 2 & \text { if } n_{1}=2 \text { and } n_{2} \geqslant 3(t=2), \\ \sum_{i=1}^{t-1} n_{i}-1 & \text { otherwise. }\end{cases}
$$

Theorem 126 (Hattingh and Plummer [80], 2008). For a tree $T$ of order $n(\geqslant 4), T \not K_{1, n-1}$ if and only if $b_{R}(T)=1$.

Theorem 126 shows that the restrained bondage number of a tree can be computed in constant time. However, the decision problem for $b_{\mathrm{R}}(G)$ is NP-complete even for bipartite graphs.

Problem 5. Consider the decision problem:

Restrained Bondage Problem

Instance: a graph $G$ and a positive integer $b$.

Question: is $b_{\mathrm{R}}(G) \leqslant b$ ?

Theorem 127 (Hattingh and Plummer [80], 2008). The restrained bondage problem is NP-complete, even for bipartite graphs.

Consequently, in view of its computational hardness, it is significative to establish some sharp bounds on the restrained bondage number of a graph in terms of other graphic parameters.

Theorem 128 (Hattingh and Plummer [80], 2008). For any graph $G$ with $\delta(G) \geqslant 2$,

$$
b_{R}(G) \leqslant \min _{x y \in E(G)}\left\{d_{G}(x)+d_{G}(y)-2\right\}
$$

Corollary 129. $b_{R}(G) \leqslant \Delta(G)+\delta(G)-2$ for any graph $G$ with $\delta(G) \geqslant 2$.

Notice that the bounds stated in Theorem 128 and Corollary 129 are sharp. Indeed the class of cycles whose orders are congruent to $1,2(\bmod 3)$ have a restrained bondage number achieving these bounds (see Theorem 125).

Theorem 128 is an analogue of Theorem 14. A quite natural problem is whether or not, for restricted bondage number, there are analogues of Theorem 16, Theorem 18, Theorem 29, and so on. Indeed, we should consider all results for the bondage number whether or not there are analogues for restricted bondage number.

Theorem 130 (Hattingh and Plummer [80], 2008). For any graph $G$ with $\gamma_{R}(G)=2$,

$$
b_{R}(G) \leqslant \Delta(G)+1 \text {. }
$$

We now consider the relation between $b_{R}(G)$ and $b(G)$.

Theorem 131 (Hattingh and Plummer [80], 2008). If $\gamma_{R}(G)=$ $\gamma(G)$ for some graph $G$, then $b_{R}(G) \leqslant b(G)$.

Corollary 129 and Theorem 131 provide a possibility to attack Conjecture 73 by characterizing planar graphs with $\gamma_{R}=\gamma$ and $b_{\mathrm{R}}=b$ when $3 \leqslant \Delta \leqslant 6$.

However, we do not have $b_{\mathrm{R}}(G)=b(G)$ for any graph $G$ even if $\gamma_{R}(G)=\gamma(G)$. Observe that $\gamma_{R}\left(K_{3}\right)=\gamma\left(K_{3}\right)$, yet $b_{R}\left(K_{3}\right)=1$ and $b\left(K_{3}\right)=2$. We still may not claim that $b_{R}(G)=b(G)$ even in the case that every $\gamma$-set is a $\gamma_{R}$-set. The example $K_{3}$ again demonstrates this.

Theorem 132 (Hattingh and Plummer [80], 2008). There is an infinite class of graphs in which each graph $G$ satisfies $b(G)<$ $b_{R}(G)$.

In fact, such an infinite class of graphs can be obtained as follows. Let $H$ be a connected graph, $\mathrm{BC}(H)$ a graph obtained from $H$ by attaching $\ell(\geqslant 2)$ vertices of degree 1 to each vertex in $H$, and $\mathscr{B}=\{G: G=\mathrm{BC}(H)$ for some graph $H$ such that $\delta(H) \geqslant 2\}$. By Theorem 3 , we can easily check that $b(G)=1<2 \leqslant \min \{\delta(H), \ell\}=b_{\mathrm{R}}(G)$ for any $G \in \mathscr{B}$. Moreover, there exists a graph $G \in \mathscr{B}$ such that $b_{\mathrm{R}}(G)$ can be much larger than $b(G)$, which is stated as the following theorem.

Theorem 133 (Hattingh and Plummer [80], 2008). For each positive integer $k$, there is a graph $G$ such that $k=b_{R}(G)-b(G)$.

Combining Theorem 133 with Theorem 132, we have the following corollary.

Corollary 134. The bondage number and the restrained bondage number are unrelated.

Very recently, Jafari Rad et al. [81] have considered the total restrained bondage in graphs based on both total dominating sets and restrained dominating sets and obtained several properties, exact values, and bounds for the total restrained bondage number of a graph.

A restrained dominating set $S$ of a graph $G$ without isolated vertices is called to be a total restrained dominating set if $S$ is a total dominating set. The total restrained domination number of $G$, denoted by $\gamma_{\mathrm{TR}}(G)$, is the minimum cardinality of a total restrained dominating set of $G$. For references on this domination in graphs, see [3, 61, 82-85]. The total restrained bondage number $b_{\mathrm{TR}}(G)$ of a graph $G$ with no isolated vertex, is the cardinality of a smallest set of edges $B \subseteq$ $E(G)$ for which $G-B$ has no isolated vertex and $\gamma_{\mathrm{TR}}(G-B)>$ $\gamma_{\mathrm{TR}}(G)$. In the case that there is no such subset $B$, we define $b_{\mathrm{TR}}(G)=\infty$.

Ma et al. [84] determined that $\gamma_{\mathrm{TR}}\left(P_{n}\right)=n-2\lfloor(n-2) / 4\rfloor$ for $n \geqslant 3, \gamma_{\mathrm{TR}}\left(C_{n}\right)=n-2\lfloor n / 4\rfloor$ for $n \geqslant 3, \gamma_{\mathrm{TR}}\left(K_{3}\right)=$ 3 and $\gamma_{\mathrm{TR}}\left(K_{n}\right)=2$ for $n \neq 3$, and $\gamma_{\mathrm{TR}}\left(K_{1, n}\right)=1+n$ and $\gamma_{\mathrm{TR}}\left(K_{m, n}\right)=2$ for $2 \leqslant m \leqslant n$. According to these results, Jafari Rad et al. [81] determined the exact values of total restrained bondage numbers for the corresponding graphs. 
Theorem 135 (Jafari Rad et al. [81]). For an integer $n$,

$$
\begin{gathered}
b_{\mathrm{TR}}\left(P_{n}\right)= \begin{cases}\infty & \text { if } 2 \leqslant n \leqslant 5, \\
1, & \text { if } n \geqslant 5\end{cases} \\
b_{\mathrm{TR}}\left(C_{n}\right)= \begin{cases}\infty & \text { if } n=3, \\
1, & \text { if } 3<n, n \equiv 0,1(\bmod 4) \\
2, & \text { if } 3<n, n \equiv 2,3(\bmod 4)\end{cases} \\
b_{\mathrm{TR}}\left(W_{n}\right)=2 \quad \text { for } n \geqslant 6, \\
b_{\mathrm{TR}}\left(K_{n}\right)=n-1 \quad \text { for } n \geqslant 4, \\
b_{\mathrm{TR}}\left(K_{m, n}\right)=m-1 \quad \text { for } 2 \leqslant m \leqslant n .
\end{gathered}
$$

$b_{\mathrm{TR}}\left(K_{n}\right)=n-1$ shows the fact that for any integer $n \geqslant 3$ there exists a connected graph, namely, $K_{n+1}$ such that $b_{\mathrm{TR}}\left(K_{n+1}\right)=$ $n$; that is, the total restrained bondage number is unbounded from the above.

A vertex is said to be a support vertex if it is adjacent to a vertex of degree one. Let $\mathscr{A}$ be the family of all connected graphs such that $G$ belongs to $\mathscr{A}$ if and only if every edge of $G$ is incident to a support vertex or $G$ is a cycle of length three.

Proposition 136 (Cyman and Raczek [82], 2006). For a connected graph $G$ of order $n, \gamma_{\mathrm{TR}}(G)=n$ if and only if $G \in \mathscr{A}$.

Hence, if $G \in \mathscr{A}$, then the removal of any subset of edges of $G$ cannot increase the total restrained domination number of $G$ and thus $b_{\mathrm{TR}}(G)=\infty$. When $G \notin \mathscr{A}$, a result similar to Theorem 6 is obtained.

Theorem 137 (Jafari Rad et al. [81]). For any tree $T \notin \mathscr{A}$, $b_{\mathrm{TR}}(T) \leqslant 2$. This bound is sharp.

In the same paper, the authors provided a characterization for trees $T \notin \mathscr{A}$ with $b_{\mathrm{TR}}(T)=1$ or 2 . The following result is similar to Observation 1 .

Observation 3 (Jafari Rad et al. [81]). If $k$ edges can be removed from a graph $G$ to obtain a graph $H$ without an isolated vertex and with $b_{\mathrm{TR}}(H)=t$, then $b_{\mathrm{TR}}(G) \leqslant k+t$.

Applying Theorem 137 and Observation 3, Jafari Rad et al. characterized all connected graphs $G$ with $b_{\mathrm{TR}}(G)=\infty$.

Theorem 138 (Jafari Rad et al. [81]). For a connected graph $G$, $b_{\mathrm{TR}}(G)=\infty$ if and only if $G \in \mathscr{A}$.

8.4. Other Conditional Bondage Numbers. A subset $I \subseteq V(G)$ is called an independent set if no two vertices in $I$ are adjacent in $G$. The maximum cardinality among all independent sets is called the independence number of $G$, denoted by $\alpha(G)$.

A dominating set $S$ of a graph $G$ is called to be independent if $S$ is an independent set of $G$. The minimum cardinality among all independent dominating set is called the independence domination number of $G$ and denoted by $\gamma_{I}(G)$.
Since an independent dominating set is not only a dominating set but also an independent set, $\gamma(G) \leqslant \gamma_{I}(G) \leqslant$ $\alpha(G)$ for any graph $G$.

It is clear that a maximal independent set is certainly a dominating set. Thus, an independent set is maximal if and only if it is an independent dominating set, and so $\gamma_{I}(G)$ is the minimum cardinality among all maximal independent sets of $G$. This graph-theoretical invariant has been well studied in the literature; see, for example, Haynes et al. [3] for early results, Goddard and Henning [86] for recent results on independent domination in graphs.

In 2003, Zhang et al. [87] defined the independence bondage number $b_{I}(G)$ of a nonempty graph $G$ to be the minimum cardinality among all subsets $B \subseteq E(G)$ for which $\gamma_{I}(G-B)>\gamma_{I}(G)$. For some ordinary graphs, their independence domination numbers can be easily determined, and so independence bondage numbers have been also determined. Clearly, $b_{I}\left(K_{n}\right)=1$ if $n \geqslant 2$.

Theorem 139 (Zhang et al. [87], 2003). For any integer $n \geqslant 4$,

$$
\begin{gathered}
b_{I}\left(C_{n}\right)= \begin{cases}1 & \text { if } n \equiv 1(\bmod 2), \\
2 & \text { if } n \equiv 0(\bmod 2),\end{cases} \\
b_{I}\left(P_{n}\right)= \begin{cases}1 & \text { if } n \equiv 0(\bmod 2), \\
2 & \text { if } n \equiv 1(\bmod 2),\end{cases} \\
b_{I}\left(K_{m, n}\right)=n \quad \text { for } m \leqslant n .
\end{gathered}
$$

Apart from the above-mentioned results, as far as we find no other results on the independence bondage number in the present literature, we never knew much of any result on this parameter for a tree.

A subset $S$ of $V(G)$ is called an equitable dominating set if for every $y \in V(G-S)$ there exists a vertex $x \in S$ such that $x y \in E(G)$ and $\left|d_{G}(x)-d_{G}(y)\right| \leqslant 1$ proposed by Dharmalingam [88]. The minimum cardinality of such a dominating set is called the equitable domination number and is denoted by $\gamma_{E}(G)$. Deepak et al. [89] defined the equitable bondage number $b_{E}(G)$ of a graph $G$ to be the cardinality of a smallest set $B \subseteq E(G)$ of edges for which $\gamma_{E}(G-B)>\gamma_{E}(G)$ and determined $b_{E}(G)$ for some special graphs.

Theorem 140 (Deepak et al. [89], 2011). For any integer $n \geqslant 2$,

$$
\begin{gathered}
b_{E}\left(K_{n}\right)=\left\lceil\frac{n}{2}\right\rceil, \\
b_{E}\left(K_{m, n}\right)=m \quad \text { for } 0 \leqslant n-m \leqslant 1, \\
b_{E}\left(C_{n}\right)= \begin{cases}3 & \text { if } n \equiv 1(\bmod 3) \\
2 & \text { otherwise, }\end{cases} \\
b_{E}\left(P_{n}\right)= \begin{cases}2 & \text { if } n \equiv 1(\bmod 3) \\
1 & \text { otherwise, }\end{cases} \\
b_{E}(T) \leqslant 2 \quad \text { for any nontrivial tree } T, \\
b_{E}(G) \leqslant n-1 \text { for any connected graph } G \text { of order } n .
\end{gathered}
$$


As far as we know, there are no more results on the equitable bondage number of graphs in the present literature.

There are other variations of the normal domination by adding restricted conditions to the normal dominating set. For example, the connected domination set of a graph $G$, proposed by Sampathkumar and Walikar [90], is a dominating set $S$ such that the subgraph induced by $S$ is connected. The problem of finding a minimum connected domination set in a graph is equivalent to the problem of finding a spanning tree with maximum number of vertices of degree one [91], and has some important applications in wireless networks [92]; thus, it has received much research attention. However, for such an important domination, there is no result on its bondage number. We believe that the topic is of significance for future research.

\section{Generalized Bondage Numbers}

There are various generalizations of the classical domination, such as $p$-domination, distance domination, fractional domination, Roman domination, and rainbow domination. Every such a generalization can lead to a corresponding bondage. In this section, we introduce some of them.

9.1. p-Bondage Numbers. In 1985, Fink and Jacobson [93] introduced the concept of $p$-domination. Let $p$ be a positive integer. A subset $S$ of $V(G)$ is a $p$-dominating set of $G$ if $\left|S \cap N_{G}(y)\right| \geqslant p$ for every $y \in \bar{S}$. The $p$-domination number $\gamma_{p}(G)$ is the minimum cardinality among all $p$-dominating sets of $G$. Any $p$-dominating set of $G$ with cardinality $\gamma_{p}(G)$ is called a $\gamma_{p}$-set of $G$. Note that a $\gamma_{1}$-set is a classic minimum dominating set. Notice that every graph has a $p$-dominating set since the vertex-set $V(G)$ is such a set. We also note that a 1dominating set is a dominating set, and so $\gamma(G)=\gamma_{1}(G)$. The $p$-domination number has received much research attention; see a state-of-the-art survey article by Chellali et al. [94].

It is clear from definition that every $p$-dominating set of a graph certainly contains all vertices of degree at most $p-1$. By this simple observation, to avoid the trivial case occurrence, we always assume that $\Delta(G) \geqslant p$. For $p \geqslant 2$, Lu et al. [95] gave a constructive characterization of trees with unique minimum $p$-dominating sets.

Recently, $\mathrm{Lu}$ and $\mathrm{Xu}$ [96] have introduced the concept to the $p$-bondage number of $G$, denoted by $b_{p}(G)$, as the minimum cardinality among all sets of edges $B \subseteq E(G)$ such that $\gamma_{p}(G-B)>\gamma_{p}(G)$. Clearly, $b_{1}(G)=b(G)$.

$\mathrm{Lu}$ and $\mathrm{Xu}$ [96] established a lower bound and an upper bound on $b_{p}(T)$ for any integer $p \geqslant 2$ and any tree $T$ with $\Delta(T) \geqslant p$ and characterized all trees achieving the lower bound and the upper bound, respectively.

Theorem 141 (Lu and $\mathrm{Xu}[96], 2011)$. For any integer $p \geqslant 2$ and any tree $T$ with $\Delta(T) \geqslant p$,

$$
1 \leqslant b_{p}(T) \leqslant \Delta(T)-p+1 .
$$

Let $S$ be a given subset of $V(G)$ and, for any $x \in S$, let

$$
N_{p}(x, S, G)=\left\{y \in \bar{S} \cap N_{G}(x):\left|N_{G}(y) \cap S\right|=p\right\} .
$$

Then, all trees achieving the lower bound 1 can be characterized as follows.

Theorem 142 ( $\mathrm{Lu}$ and $\mathrm{Xu}[96], 2011)$. Let $T$ be a tree with $\Delta(T) \geqslant p \geqslant 2$. Then $b_{p}(T)=1$ if and only if for any $\gamma_{p}$-set $S$ of $T$ there exists an edge $x y \in(S, \bar{S})$ such that $y \in N_{p}(x, S, T)$

The notation $S(a, b)$ denotes a double star obtained by adding an edge between the central vertices of two stars $K_{1, a-1}$ and $K_{1, b-1}$. And the vertex with degree $a$ (resp., $\left.b\right)$ in $S(a, b)$ is called the $L$-central vertex (resp., $R$-central vertex) of $S(a, b)$ :

To characterize all trees attaining the upper bound given in Theorem 141, we define three types of operations on a tree $T$ with $\Delta(T)=\Delta \geqslant p+1$ :

Type 1: attach a pendant edge to a vertex $y$ with $d_{T}(y) \leqslant p-$ 2 in $T$.

Type 2: attach a star $K_{1, \Delta-1}$ to a vertex $y$ of $T$ by joining its central vertex to $y$, where $y$ in a $\gamma_{p}$-set of $T$ and $d_{T}(y) \leqslant \Delta-1$.

Type 3: attach a double star $S(p, \Delta-1)$ to a pendant vertex $y$ of $T$ by coinciding its $R$-central vertex with $y$, where the unique neighbor of $y$ is in a $\gamma_{p}$-set of $T$.

Let $\mathscr{B}=\left\{T\right.$ : a tree obtained from $K_{1, \Delta}$ or $S(p, \Delta)$ by a finite sequence of operations of Types 1,2 , and 3$\}$.

Theorem 143 ( $\mathrm{Lu}$ and $\mathrm{Xu}$ [96], 2011). A tree with the maximum $\Delta \geqslant p+1$ has $p$-bondage number $\Delta-p+1$ if and only if it belongs to $\mathscr{B}$.

Theorem $144\left(\mathrm{Lu}\right.$ and $\mathrm{Xu}$ [97], 2012). Let $G_{m, n}=P_{m} \times P_{n}$. Then

$$
\begin{aligned}
& b_{2}\left(G_{2, n}\right)=1 \quad \text { for } n \geqslant 2, \\
& b_{2}\left(G_{3, n}\right)=\left\{\begin{array}{ll}
2 & \text { if } n \equiv 1(\bmod 3), \\
1 & \text { otherwise. }
\end{array} \text { for } n \geqslant 2,\right. \\
& b_{2}\left(G_{4, n}\right)=\left\{\begin{array}{ll}
1 & \text { if } n \equiv 3(\bmod 4), \\
2 & \text { otherwise. }
\end{array} \text { for } n \geqslant 7 .\right.
\end{aligned}
$$

Recently, Krzywkowski [98] has proposed the concept of the nonisolating 2-bondage of a graph. The nonisolating 2-bondage number of a graph $G$, denoted by $b_{2}^{\prime}(G)$, is the minimum cardinality among all sets of edges $B \subseteq E(G)$ such that $G-B$ has no isolated vertices and $\gamma_{2}(G-B)>\gamma_{2}(G)$. If for every $B \subseteq E(G)$, either $\gamma_{2}(G-B)=\gamma_{2}(G)$ or $G-B$ has isolated vertices, then define $b_{2}^{\prime}(G)=0$, and say that $G$ is a 2nonisolatedly strongly stable graph. Krzywkowski presented some basic properties of nonisolating 2-bondage in graphs, showed that for every nonnegative integer $k$ there exists a tree $T$ such $b_{2}^{\prime}(T)=k$, characterized all 2-non-isolatedly strongly stable trees, and determined $b_{2}^{\prime}(G)$ for several special graphs. 
Theorem 145 (Krzywkowski [98], 2012). For any positive integers $n$ and $m$ with $m \leqslant n$,

$$
\begin{aligned}
& b_{2}^{\prime}\left(K_{n}\right)= \begin{cases}0 & \text { if } n=1,2,3 \\
\left\lfloor\frac{2 n}{3}\right\rfloor & \text { otherwise, }\end{cases} \\
& b_{2}^{\prime}\left(P_{n}\right)= \begin{cases}0 & \text { if } n=1,2,3 \\
1 & \text { otherwise, }\end{cases} \\
& b_{2}^{\prime}\left(C_{n}\right)= \begin{cases}0 & \text { if } n=3 \\
1 & \text { if } n \text { is even } \\
2 & \text { if } n \text { is odd, }\end{cases} \\
& b_{2}^{\prime}\left(K_{m, n}\right)= \begin{cases}3 & \text { if } m=n=3 \\
1 & \text { if } m=n=4 \\
2 & \text { otherwise. }\end{cases}
\end{aligned}
$$

9.2. Distance Bondage Numbers. A subset $S$ of vertices of a graph $G$ is said to be a distance $k$-dominating set for $G$ if every vertex in $G$ not in $S$ is at distance at most $k$ from some vertex of $S$. The minimum cardinality of all distance $k$-dominating sets is called the distance $k$-domination number of $G$ and denoted by $\gamma_{k}(G)$ (do not confuse with the above-mentioned $\gamma_{p}(G)$ !). When $k=1$, a distance 1 -dominating set is a normal dominating set, and so $\gamma_{1}(G)=\gamma(G)$ for any graph $G$. Thus, the distance $k$-domination is a generalization of the classical domination.

The relation between $\gamma_{k}$ and $\alpha_{k}$ (the $k$-independence number, defined in Section 2.2) for a tree obtained by Meir and Moon [14], who proved that $\gamma_{k}(T)=\alpha_{2 k}(T)$ for any tree $T$ (a special case for $k=1$ is stated in Proposition 10). The further research results can be found in Henning et al. [99], Tian and $\mathrm{Xu}$ [100-103], and Liu et al. [104].

In 1998, Hartnell et al. [15] defined the distance $k$-bondage number of $G$, denoted by $b_{k}(G)$, to be the cardinality of a smallest subset $B$ of edges of $G$ with the property that $\gamma_{k}(G-$ $B)>\gamma_{k}(G)$. From Theorem 6 , it is clear that if $T$ is a nontrivial tree, then $1 \leqslant b_{1}(T) \leqslant 2$. Hartnell et al. [15] generalized this result to any integer $k \geqslant 1$.

Theorem 146 (Hartnell et al. [15], 1998). For every nontrivial tree $T$ and positive integer $k, 1 \leqslant b_{k}(T) \leqslant 2$.

Hartnell et al. [15] and Topp and Vestergaard [13] also characterized the trees having distance $k$-bondage number 2 . In particular, the class of trees for which $b_{1}(T)=2$ are just those which have a unique maximum 2 -independent set (see Theorem 11).

Since, when $k=1$, the distance 1 -bondage number $b_{1}(G)$ is the classical bondage number $b(G)$, Theorem 12 gives the NP-hardness of deciding the distance $k$-bondage number of general graphs.

Theorem 147. Given a nonempty undirected graph $G$ and positive integers $k$ and $b$ with $b \leqslant \varepsilon(G)$, determining wether or not $b_{k}(G) \leqslant b$ is NP-hard.
For a vertex $x$ in $G$, the open $k$-neighborhood $N_{k}(x)$ of $x$ is defined as $N_{k}(x)=\left\{y \in V(G): 1 \leqslant d_{G}(x, y) \leq k\right\}$. The closed $k$-neighborhood $N_{k}[x]$ of $x$ in $G$ is defined as $N_{k}(x) \cup\{x\}$. Let

$$
\Delta_{k}(G)=\max \left\{\left|N_{k}(x)\right|: \text { for any } x \in V(G)\right\} .
$$

Clearly, $\Delta_{1}(G)=\Delta(G)$. The $k$ th power of a graph $G$ is the graph $G^{k}$ with vertex-set $V\left(G^{k}\right)=V(G)$ and edge-set $E\left(G^{k}\right)=$ $\left\{x y: 1 \leqslant d_{G}(x, y) \leqslant k\right\}$. The following lemma holds directly from the definition of $G^{k}$.

Proposition 148 (Tian and $\mathrm{Xu}[101]) . \Delta\left(G^{k}\right)=\Delta_{k}(G)$ and $\gamma\left(G^{k}\right)=\gamma_{k}(G)$ for any graph $G$ and each $k \geqslant 1$.

A graph $G$ is $k$-distance domination-critical, or $\gamma_{k}$-critical for short, if $\gamma_{k}(G-x)<\gamma_{k}(G)$ for every vertex $x$ in $G$, proposed by Henning et al. [105]

Proposition 149 (Tian and Xu [101]). For each $k \geqslant 1$, a graph $G$ is $\gamma_{k}$-critical if and only if $G^{k}$ is $\gamma_{k}$-critical.

By the above facts, we suggest the following worthwhile problem.

Problem 6. Can we generalize the results on the bondage for $G$ to $G^{k}$ ? In particular, do the following propositions hold?

(a) $b\left(G^{k}\right)=b_{k}(G)$ for any graph $G$ and each $k \geqslant 1$.

(b) $b\left(G^{k}\right) \leqslant \Delta_{k}(G)$ if $G$ is not $\gamma_{k}$-critical.

Let $k$ and $p$ be positive integers. A subset $S$ of $V(G)$ is defined to be a $(k, p)$-dominating set of $G$ if, for any vertex $x \in \bar{S},\left|N_{k}(x) \cap S\right| \geqslant p$. The $(k, p)$-domination number of $G$, denoted by $\gamma_{k, p}(G)$, is the minimum cardinality among all $(k, p)$-dominating sets of $G$. Clearly, for a graph $G$, a $(1,1)$-dominating set is a classical dominating set, a $(k, 1)$ dominating set is a distance $k$-dominating set, and a $(1, p)$ dominating set is the above-mentioned $p$-dominating set. That is, $\gamma_{1,1}(G)=\gamma(G), \gamma_{k, 1}(G)=\gamma_{k}(G)$, and $\gamma_{1, p}(G)=\gamma_{p}(G)$.

The concept of $(k, p)$-domination in a graph $G$ is a generalized domination which combines distance $k$-domination and $p$-domination in $G$. So, the investigation of $(k, p)$ domination of $G$ is more interesting and has received the attention of many researchers; see, for example, [106-109].

More general, $\mathrm{Li}$ and $\mathrm{Xu}$ [110] proposed the concept of the $(\ell, w)$-domination number of a $w$-connected graph. A subset $S$ of $V(G)$ is defined to be an $(\ell, w)$-dominating set of $G$ if, for any vertex $x \in \bar{S}$, there are $w$ internally disjoint paths of length at most $\ell$ from $x$ to some vertex in $S$. The $(\ell, w)$ domination number of $G$, denoted by $\gamma_{\ell, w}(G)$, is the minimum cardinality among all $(\ell, w)$-dominating sets of $G$. Clearly, $\gamma_{k, 1}(G)=\gamma_{k}(G)$ and $\gamma_{1, p}(G)=\gamma_{p}(G)$.

It is quite natural to propose the concept of bondage number for $(k, p)$-domination or $(\ell, w)$-domination. However, as far as we know, none has proposed this concept until today. This is a worthwhile topic for further research. 
9.3. Fractional Bondage Numbers. If $\sigma$ is a function mapping the vertex-set $V$ into some set of real numbers, then for any subset $S \subseteq V$; let $\sigma(S)=\sum_{x \in S} \sigma(x)$. Also let $|\sigma|=\sigma(V)$.

A real-value function $\sigma: V \rightarrow[0,1]$ is a dominating function of a graph $G$ if for every $x \in V(G), \sigma\left(N_{G}[x]\right) \geqslant 1$. Thus, if $S$ is a dominating set of $G, \sigma$ is a function, where

$$
\sigma(x)= \begin{cases}1 & \text { if } x \in S \\ 0 & \text { otherwise }\end{cases}
$$

then $\sigma$ is a dominating function of $G$. The fractional domination number of $G$, denoted by $\gamma_{F}(G)$, is defined as follows.

$$
\gamma_{F}(G)=\min \{|\sigma|: \sigma \text { is a domination function of } G\} .
$$

The $\gamma_{F}$-bondage number of $G$, denoted by $b_{F}(G)$, is defined as the minimum cardinality of a subset $B \subseteq E$ whose removal results in $\gamma_{F}(G-B)>\gamma_{F}(G)$.

Hedetniemi et al. [111] are the first to study fractional domination although Farber [112] introduced the idea indirectly. The concept of the fractional domination number was proposed by Domke and Laskar [113] in 1997. The fractional domination numbers for some ordinary graphs are determined.

Proposition 150 (Domke et al. [114], 1998; Domke and Laskar [113], 1997). (a) If $G$ is a k-regular graph with order $n$, then $\gamma_{F}(G)=n / k+1$;

(b) $\gamma_{F}\left(C_{n}\right)=n / 3, \gamma_{F}\left(P_{n}\right)=\lceil n / 3\rceil, \gamma_{F}\left(K_{n}\right)=1$;

(c) $\gamma_{F}(G)=1$ if and only if $\Delta(G)=n-1$;

(d) $\gamma_{F}(T)=\gamma(T)$ for any tree $T$;

(e) $\gamma_{F}\left(K_{n, m}\right)=(n(m+1)+m(n+1)) /(n m-1)$, where $\max \{n, m\}>1$.

The assertions (a)-(d) are due to Domke et al. [114] and the assertion (e) is due to Domke and Laskar [113]. According to these results, Domke and Laskar [113] determined the $\gamma_{F^{-}}$ bondage numbers for these graphs.

Theorem 151 (Domke and Laskar [113], 1997). $b_{F}\left(K_{n}\right)=$ $\lceil n / 2\rceil ; b_{F}\left(K_{n, m}\right)=1$ where $\max \{n, m\}>1$,

$$
\begin{aligned}
& b_{F}\left(C_{n}\right)= \begin{cases}2 & \text { if } n \equiv 0(\bmod 3), \\
1 & \text { otherwise, }\end{cases} \\
& b_{F}\left(P_{n}\right)= \begin{cases}2 & \text { if } n \equiv 1(\bmod 3), \\
1 & \text { otherwise, }\end{cases}
\end{aligned}
$$

It is easy to see that for any tree $T, b_{F}(T) \leqslant 2$. In fact, since $\gamma_{F}(T)=\gamma(T)$ for any tree $T, \gamma_{F}\left(T^{\prime}\right)=\gamma\left(T^{\prime}\right)$ for any subgraph $T^{\prime}$ of $T$. It follows that

$$
\begin{aligned}
b_{F}(T) & =\min \left\{B \subseteq E(T): \gamma_{F}(T-B)>\gamma_{F}(T)\right\} \\
& =\min \{B \subseteq E(T): \gamma(T-B)>\gamma(T)\} \\
& =b(T) \leqslant 2 .
\end{aligned}
$$
yet.
9.4. Roman Bondage Numbers. A Roman dominating function on a graph $G$ is a labeling $f: V \rightarrow\{0,1,2\}$ such that every vertex with label 0 has at least one neighbor with label 2. The weight of a Roman dominating function $f$ on $G$ is the value

$$
f(G)=\sum_{u \in V(G)} f(u) .
$$

The minimum weight of a Roman dominating function on a graph $G$ is called the Roman domination number of $G$, denoted by $\gamma_{\mathrm{RM}}(G)$.

A Roman dominating function $f: V \rightarrow\{0,1,2\}$ can be represented by the ordered partition $\left(V_{0}, V_{1}, V_{2}\right)$ (or $\left(V_{0}^{f}, V_{1}^{f}, V_{2}^{f}\right)$ to refer to $\left.f\right)$ of $V$, where $V_{i}=\{v \in V \mid f(v)=i\}$. In this representation, its weight $f(G)=\left|V_{1}\right|+2\left|V_{2}\right|$. It is clear that $V_{1}^{f} \cup V_{2}^{f}$ is a dominating set of $G$, called the Roman dominating set, denoted by $D_{\mathrm{R}}^{f}=\left(V_{1}, V_{2}\right)$. Since $V_{1}^{f} \cup V_{2}^{f}$ is a dominating set when $f$ is a Roman dominating function, and since placing weight 2 at the vertices of a dominating set yields a Roman dominating function, in [115], it is observed that

$$
\gamma(G) \leqslant \gamma_{\mathrm{RM}}(G) \leqslant 2 \gamma(G) .
$$

A graph $G$ is called to be Roman if $\gamma_{\mathrm{RM}}(G)=2 \gamma(G)$.

The definition of the Roman domination function is proposed implicitly by Stewart [116] and ReVelle and Rosing [117]. Roman dominating numbers have been deeply studied. In particular, Bahremandpour et al. [118] showed that the problem determining the Roman domination number is NPcomplete even for bipartite graphs.

Let $G$ be a graph with maximum degree at least two. The Roman bondage number $b_{\mathrm{RM}}(G)$ of $G$ is the minimum cardinality of all sets $E^{\prime} \subseteq E$ for which $\gamma_{\mathrm{RM}}\left(G-E^{\prime}\right)>$ $\gamma_{\mathrm{RM}}(G)$. Since in study of Roman bondage number the assumption $\Delta(G) \geqslant 2$ is necessary, we always assume that when we discuss $b_{\mathrm{RM}}(G)$, all graphs involved satisfy $\Delta(G) \geqslant$ 2. The Roman bondage number $b_{\mathrm{RM}}(G)$ is introduced by Jafari Rad and Volkmann in [119].

Recently, Bahremandpour et al. [118] have shown that the problem determining the Roman bondage number is NPhard even for bipartite graphs.

Problem 7. Consider the decision problem:

Roman Bondage Problem

Instance: a nonempty bipartite graph $G$ and a positive integer $k$.

Question: is $b_{\mathrm{RM}}(G) \leqslant k$ ?

Theorem 152 (Bahremandpour et al. [118], 2013). The Roman bondage problem is NP-hard even for bipartite graphs.

The exact value of $b_{\mathrm{RM}}(G)$ is known only for a few family of graphs including the complete graphs, cycles, and paths. 
Theorem 153 (Jafari Rad and Volkmann [119], 2011). For any positive integers $n$ and $m$ with $m \leqslant n$,

$$
\begin{aligned}
& b_{\mathrm{RM}}\left(P_{n}\right)= \begin{cases}2, & \text { if } n \equiv 2(\bmod 3), \\
1, & \text { otherwise, }\end{cases} \\
& b_{\mathrm{RM}}\left(C_{n}\right)= \begin{cases}3, & \text { if } n=2(\bmod 3), \\
2, & \text { otherwise, }\end{cases} \\
& b_{\mathrm{RM}}\left(K_{n}\right)=\left\{\begin{array}{ll}
\frac{n}{2}
\end{array} \quad \text { for } n \geqslant 3,\right. \\
& b_{\mathrm{RM}}\left(K_{m, n}\right)= \begin{cases}1 & \text { if } m=1 \text { and } n \neq 1, \\
4 & \text { if } m=n=3, \\
m & \text { otherwise. }\end{cases}
\end{aligned}
$$

Lemma 154 (Cockayne et al. [115], 2004). If G is a graph of order $n$ and contains vertices of degree $n-1$, then $\gamma_{\mathrm{RM}}(G)=2$.

Using Lemma 154, the third conclusion in Theorem 153 can be generalized to more general case, which is similar to Lemma 49.

Theorem 155 (Jafari Rad and Volkmann [119], 2011). Let G be a graph with order $n \geq 3$ and the number of vertices of degree $n-1$ in $G$. If $t \geqslant 1$, then $b_{\mathrm{RM}}(G)=\lceil t / 2\rceil$.

Ebadi and PushpaLatha [120] conjectured that $b_{\mathrm{RM}}(G) \leqslant$ $n-1$ for any graph $G$ of order $n \geqslant 3$. Dehgardi et al. [121], Akbari and Qajar [122], independently, showed that this conjecture is true.

Theorem 156. $b_{R M}(G) \leqslant n-1$ for any connected graph $G$ of $\operatorname{order} n \geqslant 3$.

For a complete $t$-partite graph, we have the following result.

Theorem 157 ( $\mathrm{Hu}$ and $\mathrm{Xu}[123], 2011)$. Let $G=K_{m_{1}, m_{2}, \ldots, m_{t}}$ be a complete $t$-partite graph with $m_{1}=\cdots=m_{i}<m_{i+1} \leq \cdots \leq$ $m_{t}, t \geqslant 2$ and $n=\sum_{j=1}^{t} m_{j}$. Then

$$
b_{\mathrm{RM}}(G)= \begin{cases}\left\lceil\frac{i}{2}\right\rceil & \text { if } m_{i}=1 \text { and } n \geqslant 3, \\ 2 & \text { if } m_{i}=2 \text { and } i=1, \\ i & \text { if } m_{i}=2 \text { and } i \geqslant 2, \\ 4 & \text { if } m_{i}=3 \text { and } i=t=2, \\ n-1 & \text { if } m_{i}=3 \text { and } i=t \geqslant 3, \\ n-m_{t} & \text { if } m_{i} \geqslant 3 \text { and } m_{t} \geqslant 4 .\end{cases}
$$

Consider a complete $t$-partite graph $K_{t}(3)$ for $t \geqslant 3$, $b_{\mathrm{RM}}\left(K_{t}(3)\right)=n-1$ by Theorem 157, where $n=3 t$, which shows that this upper bound of $n-1$ on $b_{\mathrm{RM}}(G)$ in Theorem 156 is sharp. This fact and $\gamma_{R}\left(K_{t}(3)\right)=4$ give a negative answer to the following two problems posed by Dehgardi et al. [121].

Prove or Disprove: for any connected graph $G$ of order $n \geq 3$, (i) $b_{\mathrm{RM}}(G)=n-1$ if and only if $G \cong K_{3}$; (ii) $b_{\mathrm{RM}}(G) \leqslant n-$ $\gamma_{R}(G)+1$.

Note that a complete $t$-partite graph $K_{t}(3)$ is $(n-3)$ regular and $b_{\mathrm{RM}}\left(K_{t}(3)\right)=n-1$ for $t \geqslant 3$, where $n=3 t$. $\mathrm{Hu}$ and $\mathrm{Xu}$ further determined that $b_{R}(G)=n-2$ for any $(n-3)$-regular graph $G$ of order $n \leqslant 5$ except $K_{t}(3)$.

Theorem 158 (Hu and $\mathrm{Xu}[123], 2011)$. For any (n-3)-regular graph $G$ of order $n$ other than $K_{t}(3), b_{R M}(G)=n-2$ for $n \geqslant 5$.

For a tree $T$ with order $n \geqslant 3$, Ebadi and PushpaLatha [120], and Jafari Rad and Volkmann [119], independently, obtained an upper bound on $b_{\mathrm{RM}}(T)$.

Theorem 159. $b_{R M}(T) \leqslant 3$ for any tree $T$ with order $n \geqslant 3$.

Theorem 160 (Bahremandpour et al. [118], 2013). $b_{\mathrm{RM}}\left(P_{2} \times\right.$ $\left.P_{n}\right)=2$ for $n \geqslant 2$.

Theorem 161 (Jafari Rad and Volkmann [119], 2011). Let G be a graph with order at least three.

(a) If $(x, y, z)$ is a path of length 2 in $G$, then $b_{\mathrm{RM}}(G) \leqslant$ $d_{G}(x)+d_{G}(y)+d_{G}(z)-3-\left|N_{G}(x) \cap N_{G}(y)\right|$.

(b) If $G$ is connected, then $b_{\mathrm{RM}}(G) \leqslant \lambda(G)+2 \Delta(G)-3$.

Theorem 161 (b) implies $b_{\mathrm{RM}}(G) \leqslant \delta(G)+2 \Delta(G)-3$ for a connected graph $G$. Note that for a planar graph $G, \delta(G) \leqslant 5$; moreover, $\delta(G) \leqslant 3$ if the girth at least 4 , and $\delta(G) \leqslant 2$ if the girth at least 6 . These two facts show that $b_{\mathrm{RM}}(G) \leqslant 2 \Delta(G)+$ 2 for a connected planar graph $G$. Jafari Rad and Volkmann [124] improved this bound.

Theorem 162 (Jafari Rad and Volkmann [124], 2011). For any connected planar graph $G$ of order $n \geqslant 3$ with girth $g(G)$,

$b_{\mathrm{RM}}(G)$

$$
\leqslant \begin{cases}2 \Delta(G), & \\ \Delta(G)+6, & \\ \Delta(G)+5 & \text { if } G \text { contains no vertices of degree five, } \\ \Delta(G)+4 & \text { if } g(G) \geqslant 4 \\ \Delta(G)+3 & \text { if } g(G) \geqslant 5 \\ \Delta(G)+2 & \text { if } g(G) \geqslant 6 \\ \Delta(G)+1 & \text { if } g(G) \geqslant 8\end{cases}
$$

According to Theorem 157, $b_{\mathrm{RM}}\left(C_{n}\right)=3=\Delta\left(C_{n}\right)+1$ for a cycle $C_{n}$ of length $n \geqslant 8$ with $n \equiv 2(\bmod 3)$, and therefore the last upper bound in Theorem 162 is sharp, at least for $\Delta=$ 2.

Combining the fact that every planar graph $G$ with minimum degree 5 contains an edge $x y$ with $d_{G}(x)=5$ and $d_{G}(y) \in\{5,6\}$ with Theorem 161 (a), Akbari et al. [125] obtained the following result. 


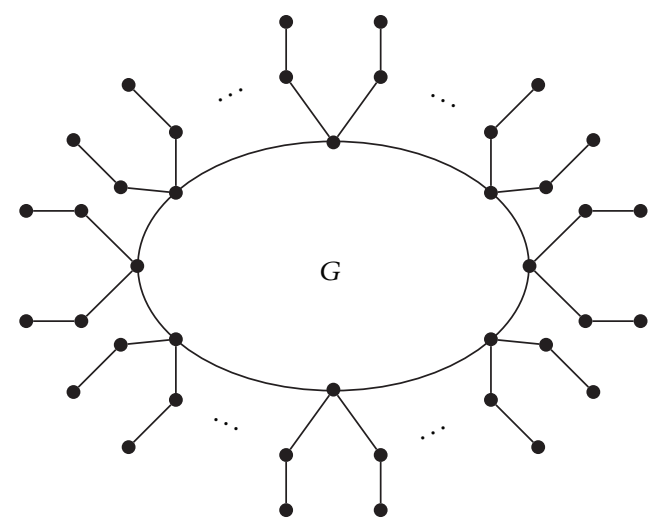

FIgURE 9: The graph $\widehat{G}$ is constructed from $G$.

Theorem 163 (Akbari et al. [125], 2012). $b_{\mathrm{RM}}(G) \leqslant 15$ for every planar graph $G$.

It remains open to show whether the bound in Theorem 163 is sharp or not. Though finding a planar graph $G$ with $b_{\mathrm{RM}}(G)=15$ seems to be difficult, Akbari et al. [125] constructed an infinite family of planar graphs with Roman bondage number equal to 7 by proving the following result.

Theorem 164 (Akbari et al. [125], 2012). Let $G$ be a graph of order $n$ and $\widehat{G}$ is the graph of order $5 n$ obtained from $G$ by attaching the central vertex of a copy of a path $P_{5}$ to each vertex of $G$ (see Figure 9). Then $\gamma_{\mathrm{RM}}(\widehat{G})=4 n$ and $b_{\mathrm{RM}}(\widehat{G})=\delta(G)+2$.

By Theorem 164, infinite many planar graphs with Roman bondage number 7 can be obtained by considering any planar graph $G$ with $\delta(G)=5$ (e.g., the icosahedron graph).

Conjecture 165 (Akbari et al. [125], 2012). The Roman bondage number of every planar graph is at most 7 .

For general bounds, the following observation is directly obtained, which is similar to Observation 1.

Observation 4. Let $G$ be a graph of order $n$ with maximum degree at least two. Assume that $H$ is a spanning subgraph obtained from $G$ by removing $k$ edges. If $\gamma_{\mathrm{RM}}(H)=\gamma_{\mathrm{RM}}(G)$, then $b_{R}(H) \leqslant b_{\mathrm{RM}}(G) \leqslant b_{\mathrm{RM}}(H)+k$.

Theorem 166 (Bahremandpour et al. [118], 2013). For any connected graph $G$ of order $n$, if $n \geqslant 3$ and $\gamma_{\mathrm{RM}}(G)=\gamma(G)+1$, then

$$
b_{\mathrm{RM}}(G) \leqslant \min \left\{b(G), n_{\Delta}\right\} \text {, }
$$

where $n_{\Delta}$ is the number of vertices with maximum degree $\Delta$ in G.

Observation 5. For any graph $G$, if $\gamma(G)=\gamma_{\mathrm{RM}}(G)$, then $b_{\mathrm{RM}}(G) \leqslant b(G)$.
Theorem 167 (Bahremandpour et al. [118], 2013). For every Roman graph $G$,

$$
b_{\mathrm{RM}}(G) \geqslant b(G) .
$$

The bound is sharp for cycles on $n$ vertices where $n \equiv 0(\bmod$ $3)$.

The strict inequality in Theorem 167 can hold, for example, $b\left(C_{3 k+2}\right)=2<3=b_{\mathrm{RM}}\left(C_{3 k+2}\right)$ by Theorem 157 .

A graph $G$ is called to be vertex Roman dominationcritical if $\gamma_{\mathrm{RM}}(G-x)<\gamma_{\mathrm{RM}}(G)$ for every vertex $x$ in $G$. If $G$ has no isolated vertices, then $\gamma_{\mathrm{RM}}(G) \leqslant 2 \gamma(G) \leqslant 2 \beta(G)$. If $\gamma_{\mathrm{RM}}(G)=2 \beta(G)$, then $\gamma_{\mathrm{RM}}(G)=2 \gamma(G)$ and hence $G$ is a Roman graph. In [30], Volkmann gave a lot of graphs with $\gamma(G)=\beta(G)$. The following result is similar to Theorem 57 .

Theorem 168 (Bahremandpour et al. [118], 2013). For any graph $G$, if $\gamma_{\mathrm{RM}}(G)=2 \beta(G)$, then

(a) $b_{\mathrm{RM}}(G) \geqslant \delta(G)$;

(b) $b_{\mathrm{RM}}(G) \geqslant \delta(G)+1$ if $G$ is a vertex Roman dominationcritical graph.

If $\gamma_{\mathrm{RM}}(G)=2$, then obviously $b_{\mathrm{RM}}(G) \leqslant \delta(G)$. So we assume $\gamma_{\mathrm{RM}}(G) \geqslant 3$. Dehgardi et al. [121] proved $b_{\mathrm{RM}}(G) \leqslant$ $\left(\gamma_{\mathrm{RM}}(G)-2\right) \Delta(G)+1$ and posed the following problem: if $G$ is a connected graph of order $n \geqslant 4$ with $\gamma_{\mathrm{RM}}(G) \geqslant 3$, then

$$
b_{\mathrm{RM}}(G) \leqslant\left(\gamma_{\mathrm{RM}}(G)-2\right) \Delta(G) \text {. }
$$

Theorem 161 (a) shows that the inequality (68) holds if $\gamma_{\mathrm{RM}}(G) \geqslant 5$. Thus, the bound in (68) is of interest only when $\gamma_{\mathrm{RM}}(G)$ is 3 or 4 .

Lemma 169 (Bahremandpour et al. [118], 2013). For any nonempty graph $G$ of order $n \geqslant 3, \gamma_{\mathrm{RM}}(G)=3$ if and only if $\Delta(G)=n-2$.

The following result shows that (68) holds for all graphs $G$ of order $n \geqslant 4$ with $\gamma_{\mathrm{RM}}(G)=3$ or 4 , which improves Theorem 161 (b).

Theorem 170 (Bahremandpour et al. [118], 2013). For any connected graph $G$ of order $n \geqslant 4$,

$$
b_{\mathrm{RM}}(G) \leq \begin{cases}\Delta(G)=n-2 & \text { if } \gamma_{\mathrm{RM}}(G)=3, \\ \Delta(G)+\delta(G)-1 & \text { if } \gamma_{\mathrm{RM}}(G)=4\end{cases}
$$

with the first equality if and only if $G \cong C_{4}$.

Recently, Akbari and Qajar [122] have proved the following result.

Theorem 171 (Akbari and Qajar [122]). For any connected $\operatorname{graph} G$ of $\operatorname{order} n \geqslant 3$,

$$
b_{\mathrm{RM}}(G) \leqslant n-\gamma_{\mathrm{RM}}(G)+5 .
$$


We conclude this section with the following problems.

Problem 8. Characterize all connected graphs $G$ of order $n \geqslant$ 3 , for which $b_{\mathrm{RM}}(G)=n-1$.

Problem 9. Prove or disprove: if $G$ is a connected graph of order $n \geqslant 3$, then

$$
b_{\mathrm{RM}}(G) \leqslant n-\gamma_{\mathrm{RM}}(G)+3 \text {. }
$$

Note that $\gamma_{R}\left(K_{t}(3)\right)=4$ and $b_{\mathrm{RM}}\left(K_{t}(3)\right)=n-1$, where $n=$ $3 t$ for $t \geqslant 3$. The upper bound on $b_{\mathrm{RM}}(G)$ given in Problem 9 is sharp if Problem 9 has positive answer.

9.5. Rainbow Bondage Numbers. For a positive integer $k$, a $k$-rainbow dominating function of a graph $G$ is a function $f$ from the vertex-set $V(G)$ to the set of all subsets of the set $\{1,2, \ldots, k\}$ such that for any vertex $x$ in $G$ with $f(x)=\emptyset$ the condition

$$
\bigcup_{y \in N_{G}(x)} f(y)=\{1,2, \ldots, k\}
$$

is fulfilled. The weight of a $k$-rainbow dominating function $f$ on $G$ is the value

$$
f(G)=\sum_{x \in V(G)}|f(x)| .
$$

The $k$-rainbow domination number of a graph $G$, denoted by $\gamma_{r k}(G)$, is the minimum weight of a $k$-rainbow dominating function $f$ of $G$.

Note that $\gamma_{r 1}(G)$ is the classical domination number $\gamma(G)$. The $k$-rainbow domination number is introduced by Brešar et al. [126]. Rainbow domination of a graph $G$ coincides with ordinary domination of the Cartesian product of $G$ with the complete graph, in particular, $\gamma_{r k}(G)=\gamma\left(G \times K_{k}\right)$ for any positive integer $k$ and any graph $G$ [126]. Hartnell and Rall [127] proved that $\gamma(T)<\gamma_{r 2}(T)$ for any tree $T$, no graph has $\gamma=\gamma_{r k}$ for $k \geqslant 3$, for any $k \geqslant 2$, and any graph $G$ of order $n$ :

$$
\min \{n, \gamma(G)+k-2\} \leqslant \gamma_{r k}(G) \leqslant k \gamma(G) .
$$

The introduction of rainbow domination is motivated by the study of paired domination in Cartesian products of graphs, where certain upper bounds can be expressed in terms of rainbow domination; that is, for any graph $G$ and any graph $H$, if $V(H)$ can be partitioned into $k \gamma_{P}$-sets, then $\gamma_{P}(G \times H) \leqslant(1 / k) v(H) \gamma_{r k}(G)$, proved by Brešar et al. [126].

Dehgardi et al. [128] introduced the concept of $k$-rainbow bondage number of graphs. Let $G$ be a graph with maximum degree at least two. The $k$-rainbow bondage number $b_{r k}(G)$ of $G$ is the minimum cardinality of all sets $B \subseteq E(G)$ for which $\gamma_{r k}(G-B)>\gamma_{r k}(G)$. Since in the study of $k$-rainbow bondage number the assumption $\Delta(G) \geqslant 2$ is necessary, we always assume that when we discuss $b_{r k}(G)$, all graphs involved satisfy $\Delta(G) \geqslant 2$.

The 2-rainbow bondage number of some special families of graphs has been determined.
Theorem 172 (Dehgardi et al. [128]). For any integer $n \geqslant 3$,

$$
\begin{aligned}
& b_{r 2}\left(P_{n}\right)=1 \quad \text { for } n \geqslant 2, \\
& b_{r 2}\left(C_{n}\right)= \begin{cases}1 & \text { if } n \equiv 0(\bmod 4), \\
2 & \text { otherwise, }\end{cases} \\
& b_{r 2}\left(K_{n, n}\right)= \begin{cases}1 & \text { if } n=2, \\
3 & \text { if } n=3, \\
6 & \text { if } n=4, \\
m & \text { if } n \geqslant 5 .\end{cases}
\end{aligned}
$$

Theorem 173 (Dehgardi et al. [128]). For any connected graph $G$ of order $n \geqslant 3, b_{r 2}(G) \leqslant \lambda(G)+2 \Delta(G)-3$.

Theorem 174 (Dehgardi et al. [128]). For any tree $T$ of order $n \geqslant 3, b_{r 2}(T) \leqslant 2$.

Theorem 175 (Dehgardi et al. [128]). If $G$ is a planar graph with maximum degree at least two, then $b_{r 2}(G) \leqslant 15$. Moreover, if the girth $g(G) \geqslant 4$, then $b_{r 2}(G) \leqslant 11$ and if $g(G) \geqslant 6$, then $b_{r 2}(G) \leqslant 9$.

Theorem 176 (Dehgardi et al. [128]). For a complete bipartite graph $K_{p, q}$, if $2 k+1 \leqslant p \leqslant q$, then $b_{r k}\left(K_{p, q}\right)=p$.

Theorem 177 (Dehgardi et al. [128]). For a complete graph $K_{n}$, if $n \geqslant k+1$, then $b_{r k}\left(K_{n}\right)=\lceil k n /(k+1)\rceil$.

The special case $k=1$ of Theorem 177 can be found in Theorem 1. The following is a simple observation similar to Observation 1.

Observation 6 (Dehgardi et al. [128]). Let $G$ be a graph with maximum degree at least two, $H$ a spanning subgraph obtained from $G$ by removing $k$ edges. If $\gamma_{r k}(H)=\gamma_{r k}(G)$, then $b_{r k}(H) \leqslant b_{r k}(G) \leqslant b_{r k}(H)+k$.

Theorem 178 (Dehgardi et al. [128]). For every graph $G$ with $\gamma_{r k}(G)=k \gamma(G), b_{r k}(G) \geqslant b(G)$.

A graph $G$ is said to be vertex k-rainbow dominationcritical if $\gamma_{r k}(G-x)<\gamma_{r k}(G)$ for every vertex $x$ in $G$. It is clear that if $G$ has no isolated vertices, then $\gamma_{r k}(G) \leqslant k \gamma(G) \leqslant$ $k \beta(G)$, where $\beta(G)$ is the vertex-covering number of $G$. Thus, if $\gamma_{r k}(G)=k \beta(G)$, then $\gamma_{r k}(G)=k \gamma(G)$.

Theorem 179 (Dehgardi et al. [128]). For any graph $G$, if $\gamma_{r k}(G)=k \beta(G)$, then

(a) $b_{r k}(G) \geqslant \delta(G)$

(b) $b_{r k}(G) \geqslant \delta(G)+1$ if $G$ is vertex $k$-rainbow dominationcritical.

As far as we know, there are no more results on the $k$ rainbow bondage number of graphs in the literature. 


\section{Results on Digraphs}

Although domination has been extensively studied in undirected graphs, it is natural to think of a dominating set as a one-way relationship between vertices of the graph. Indeed, among the earliest literatures on this subject, von Neumann and Morgenstern [129] used what is now called domination in digraphs to find solution (or kernels, which are independent dominating sets) for cooperative $n$-person games. Most likely, the first formulation of domination by Berge [130], in 1958, was given in the context of digraphs and, only some years latter by Ore [131], in 1962, for undirected graphs. Despite this history, examination of domination and its variants in digraphs has been essentially overlooked (see [4] for an overview of the domination literature). Thus, there are few, if any, such results on domination for digraphs in the literature.

The bondage number and its related topics for undirected graph have become one of major areas both in theoretical and applied researches. However, until recently, Carlson and Develin [42], Shan and Kang [132], and Huang and $\mathrm{Xu}[133,134]$ studied the bondage number for digraphs, independently. In this section, we introduce their results for general digraphs. Results for some special digraphs such as vertex-transitive digraphs are introduced in the next section.

10.1. Upper Bounds for General Digraphs. Let $G=(V, E)$ be a digraph without loops and parallel edges. A digraph $G$ is called to be asymmetric if whenever $(x, y) \in E(G)$ then $(y, x) \notin E(G)$ and to be symmetric if $(x, y) \in E(G)$ implies $(y, x) \in E(G)$. For a vertex $x$ of $V(G)$, the sets of outneighbors and in-neighbors of $x$ are, respectively, defined as $N_{G}^{+}(x)=\{y \in V(G):(x, y) \in E(G)\}$ and $N_{G}^{-}(x)=\{x \in$ $V(G):(x, y) \in E(G)\}$, the out-degree and the in-degree of $x$ are, respectively, defined as $d_{G}^{+}(x)=\left|N_{G}^{+}(x)\right|$ and $d_{G}^{-}(x)=$ $\left|N_{G}^{+}(x)\right|$. Denote the maximum and the minimum out-degree (resp., in-degree) of $G$ by $\Delta^{+}(G)$ and $\delta^{+}(G)$ (resp., $\Delta^{-}(G)$ and $\left.\delta^{-}(G)\right)$. The degree $d_{G}(x)$ of $x$ is defined as $d_{G}^{+}(x)+d_{G}^{-}(x)$, and the maximum and the minimum degrees of $G$ are denoted by $\Delta(G)$ and $\delta(G)$, respectively; that is, $\Delta(G)=\max \left\{d_{G}(x): x \in\right.$ $V(G)\}$ and $\delta(G)=\min \left\{d_{G}(x): x \in V(G)\right\}$. Note that the definitions here are different from the ones in the textbook on digraphs; see for example, $\mathrm{Xu}[1]$.

A subset $S$ of $V(G)$ is called a dominating set if $V(G)=S U$ $N_{G}^{+}(S)$, where $N_{G}^{+}(S)$ is the set of out-neighbors of $S$. Then, just as for undirected graphs, $\gamma(G)$ is the minimum cardinality of a dominating set, and the bondage number $b(G)$ is the smallest cardinality of a set $B$ of edges such that $\gamma(G-B)>$ $\gamma(G)$ if such a subset $B \subseteq E(G)$ exists. Otherwise, we put $b(G)=\infty$.

Some basic results for undirected graphs stated in Section 3 can be generalized to digraphs. For example, Theorem 18 is generalized by Carlson and Develin [42], Huang and $\mathrm{Xu}$ [133], and Shan and Kang [132], independently, as follows.

Theorem 180. Let $G$ be a digraph and $(x, y) \in E(G)$. Then $b(G) \leqslant d_{G}(y)+d_{G}^{-}(x)-\left|N_{G}^{-}(x) \cap N_{G}^{-}(y)\right|$.
Corollary 181. For a digraph $G, b(G) \leqslant \delta^{-}(G)+\Delta(G)$.

Since $\delta^{-}(G) \leqslant(1 / 2) \Delta(G)$, from Corollary 181, Conjecture 53 is valid for digraphs.

Corollary 182. For a digraph $G, b(G) \leqslant(3 / 2) \Delta(G)$.

In the case of undirected graphs, the bondage number of $G_{n}=K_{n} \times K_{n}$ achieves this bound. However, it was shown by Carlson and Develin in [42] that if we take the symmetric digraph $G_{n}$, we have $\Delta\left(G_{n}\right)=4(n-1), \gamma\left(G_{n}\right)=n$, and $b\left(G_{n}\right) \leqslant$ $4(n-1)+1=\Delta\left(G_{n}\right)+1$. So this family of digraphs cannot show that the bound in Corollary 182 is tight.

Corresponding to Conjecture 51, which is discredited for undirected graphs and is valid for digraphs, the same conjecture for digraphs can be proposed as follows.

Conjecture 183 (Carlson and Develin [42], 2006). $b(G) \leqslant$ $\Delta(G)+1$ for any digraph $G$.

If Conjecture 183 is true, then the following results shows that this upper bound is tight since $b\left(K_{n} \times K_{n}\right)=\Delta\left(K_{n} \times K_{n}\right)+1$ for a complete digraph $K_{n}$.

In 2007, Shan and Kang [132] gave some tight upper bounds on the bondage numbers for some asymmetric digraphs. For example, $b(T) \leqslant \Delta(T)$ for any asymmetric directed tree $T ; b(G) \leqslant \Delta(G)$ for any asymmetric digraph $G$ with order at least 4 and $\gamma(G) \leqslant 2$. For planar digraphs, they obtained the following results.

Theorem 184 (Shan and Kang [132], 2007). Let $G$ be a asymmetric planar digraph. Then $b(G) \leqslant \Delta(G)+2$; and $b(G) \leqslant$ $\Delta(G)+1$ if $\Delta(G) \geqslant 5$ and $d_{G}^{-}(x) \geqslant 3$ for every vertex $x$ with $d_{G}(x) \geqslant 4$.

10.2. Results for Some Special Digraphs. The exact values and bounds on $b(G)$ for some standard digraphs are determined.

Theorem 185 (Huang and $\mathrm{Xu}[133], 2006)$. For a directed cycle $C_{n}$ and a directed path $P_{n}$,

$$
\begin{aligned}
& b\left(C_{n}\right)= \begin{cases}3 & \text { if } n \text { is odd }, \\
2 & \text { if } n \text { is even, }\end{cases} \\
& b\left(P_{n}\right)= \begin{cases}2 & \text { if } n \text { is odd, } \\
1 & \text { if } n \text { is even. }\end{cases}
\end{aligned}
$$

For the de Bruijn digraph $B(d, n)$ and the Kautz digraph $K(d, n)$,

$$
\begin{gathered}
b(B(d, n))=d \quad \text { if } n \text { is odd, } \\
d \leqslant b(B(d, n)) \leqslant 2 d \quad \text { if } n \text { is even, } \\
b(K(d, n))=d+1 .
\end{gathered}
$$

Like undirected graphs, we can define the total domination number and the total bondage number. On the total bondage numbers for some special digraphs, the known results are as follows. 
Theorem 186 (Huang and $\mathrm{Xu}$ [134], 2007). For a directed cycle $C_{n}$ and a directed path $P_{n}, b_{T}\left(P_{n}\right)$ and $b_{T}\left(C_{n}\right)$ all do not exist. For a complete digraph $K_{n}$,

$$
\begin{gathered}
b_{T}\left(K_{n}\right)=\infty \quad \text { if } n=1,2, \\
b_{T}\left(K_{n}\right)=3 \quad \text { if } n=3, \\
n \leqslant b_{T}\left(K_{n}\right) \leqslant 2 n-3 \quad \text { if } n \geqslant 4 .
\end{gathered}
$$

The extended de Bruijn digraph $\operatorname{EB}\left(d, n ; q_{1}, \ldots, q_{p}\right)$ and the extended Kautz digraph $\operatorname{EK}\left(d, n ; q_{1}, \ldots, q_{p}\right)$ are introduced by Shibata and Gonda [135]. If $p=1$, then they are the de Bruijn digraph $\mathrm{B}(d, n)$ and the Kautz digraph $\mathrm{K}(d, n)$, respectively. Huang and $\mathrm{Xu}$ [134] determined their total domination numbers. In particular, their total bondage numbers for general cases are determined as follows.

Theorem 187 (Huang and $\mathrm{Xu}[134], 2007)$. If $d \geqslant 2$ and $q_{i} \geqslant 2$ for each $i=1,2, \ldots, p$, then

$$
\begin{gathered}
b_{T}\left(\operatorname{EB}\left(d, n ; q_{1}, \ldots, q_{p}\right)\right)=d^{p}-1, \\
b_{T}\left(\operatorname{EK}\left(d, n ; q_{1}, \ldots, q_{p}\right)\right)=d^{p} .
\end{gathered}
$$

In particular, for the de Bruijn digraph $B(d, n)$ and the Kautz digraph $K(d, n)$,

$$
b_{T}(\mathrm{~B}(d, n))=d-1, \quad b_{T}(\mathrm{~K}(d, n))=d .
$$

Zhang et al. [136] determined the bondage number in complete $t$-partite digraphs.

Theorem 188 (Zhang et al. [136], 2009). For a complete $t$ partite digraph $K_{n_{1}, n_{2}, \ldots, n_{t}}$, where $n_{1} \leqslant n_{2} \leqslant \cdots \leqslant n_{t}$,

$$
\begin{aligned}
& b\left(K_{\left.n_{1}, n_{2}, \ldots, n_{t}\right)}\right) \\
& = \begin{cases}m & \text { if } n_{m}=1, n_{m+1} \geqslant 2 \text { for some, } \\
\quad m(1 \leqslant m<t) \\
4 t-3 & \text { if } n_{1}=n_{2}=\cdots=n_{t}=2, \\
\sum_{i=1}^{t-1} n_{i}+2(t-1) & \text { otherwise. }\end{cases}
\end{aligned}
$$

Since an undirected graph can be thought of as a symmetric digraph, any result for digraphs has an analogy for undirected graphs in general. In view of this point, studying the bondage number for digraphs is more significant than for undirected graphs. Thus, we should further study the bondage number of digraphs and try to generalize known results on the bondage number and related variants for undirected graphs to digraphs, prove or disprove Conjecture 183. In particular, determine the exact values of $b(\mathrm{~B}(d, n))$ for an even $n$ and $b_{T}\left(K_{n}\right)$ for $n \geqslant 4$.

\section{Efficient Dominating Sets}

A dominating set $S$ of a graph $G$ is called to be efficient if for every vertex $x$ in $G,\left|N_{G}[x] \cap S\right|=1$ if $G$ is a undirected graph or $\left|N_{G}^{-}[x] \cap S\right|=1$ if $G$ is a directed graph. The concept of an efficient set was proposed by Bange et al. [137] in 1988. In the literature, an efficient set is also called a perfect dominating set (see Livingston and Stout [138] for an explanation).

From definition, if $S$ is an efficient dominating set of a graph $G$, then $S$ is certainly an independent set and every vertex not in $S$ is adjacent to exactly one vertex in $S$.

It is also clear from definition that a dominating set $S$ is efficient if and only if $\mathcal{N}_{G}[S]=\left\{N_{G}[x]: x \in S\right\}$ for the undirected graph $G$ or $\mathcal{N}_{G}^{+}[S]=\left\{N_{G}^{+}[x]: x \in S\right\}$ for the digraph $G$ is a partition of $V(G)$, where the induced subgraph by $N_{G}[x]$ or $N_{G}^{+}[x]$ is a star or an out-star with the root $x$.

The efficient domination has important applications in many areas, such as error-correcting codes, and receives much attention in the late years.

The concept of efficient dominating sets is a measure of the efficiency of domination in graphs. Unfortunately, as shown in [137], not every graph has an efficient dominating set and, moreover, it is an NP-complete problem to determine whether a given graph has an efficient dominating set. In addition, it was shown by Clark [139] in 1993 that for a wide range of $p$, almost every random undirected graph $G \in$ $\mathscr{G}(v, p)$ has no efficient dominating sets. This means that undirected graphs possessing an efficient dominating set are rare. However, it is easy to show that every undirected graph has an orientation with an efficient dominating set (see Bange et al. [140]).

In 1993, Barkauskas and Host [141] showed that determining whether an arbitrary oriented graph has an efficient dominating set is NP-complete. Even so, the existence of efficient dominating sets for some special classes of graphs has been examined; see, for example, Dejter and Serra [142] and Lee [143] for Cayley graph, Gu et al. [144] for meshes and tori, Obradović et al. [145], Huang and Xu [36], Reji Kumar and MacGillivray [146] for circulant graphs, Harary graphs and tori, and Van Wieren et al. [147] for cube-connected cycles.

In this section, we introduce results of the bondage number for some graphs with efficient dominating sets, due to Huang and $\mathrm{Xu}[36]$.

11.1. Results for General Graphs. In this subsection, we introduce some results on bondage numbers obtained by applying efficient dominating sets. We first state the two following lemmas.

Lemma 189. For any $k$-regular graph or digraph $G$ of order $n$, $\gamma(G) \geqslant n /(k+1)$, with equality if and only if $G$ has an efficient dominating set. In addition, if $G$ has an efficient dominating set, then every efficient dominating set is certainly a $\gamma$-set, and vice versa.

Let $e$ be an edge and $S$ a dominating set in $G$. We say that e supports $S$ if $e \in(S, \bar{S})$, where $(S, \bar{S})=\{(x, y) \in E(G)$ : $x \in S, y \in \bar{S}$ \}. Denote by $s(G)$ the minimum number of edges which support all $\gamma$-sets in $G$.

Lemma 190. For any graph or digraph $G, b(G) \geqslant s(G)$, with equality if $G$ is regular and has an efficient dominating set. 
A graph $G$ is called to be vertex-transitive if its automorphism group $\operatorname{Aut}(G)$ acts transitively on its vertex-set $V(G)$. A vertex-transitive graph is regular. Applying Lemmas 189 and 190, Huang and Xu obtained some results on bondage numbers for vertex-transitive graphs or digraphs.

Theorem 191 (Huang and $\mathrm{Xu}$ [36], 2008). For any vertextransitive graph or digraph $G$ of order $n$,

$$
b(G) \geqslant \begin{cases}\left\lceil\frac{n}{2 \gamma(G)}\right\rceil & \text { if } G \text { is undirected, } \\ \left\lceil\frac{n}{\gamma(G)}\right] \quad \text { if } G \text { is directed. }\end{cases}
$$

Theorem 192 (Huang and $\mathrm{Xu}[36], 2008)$. Let $G$ be a $k$-regular graph of order $n$. Then

$$
\begin{aligned}
& b(G) \leqslant k \text { if } G \text { is undirected and } n \geqslant \gamma(G)(k+1)-k+1 \\
& b(G) \leqslant k+1+\ell \text { if } G \text { is directed and } n \geqslant \gamma(G)(k+1)-\ell \\
& \text { with } 0 \leqslant \ell \leqslant k-1 .
\end{aligned}
$$

Next, we introduce a better upper bound on $b(G)$. To this aim, we need the following concept, which is a generalization of the concept of the edge-covering of a graph $G$. For $V^{\prime} \subseteq$ $V(G)$ and $E^{\prime} \subseteq E(G)$, we say that $E^{\prime}$ covers $V^{\prime}$ and call $E^{\prime}$ an edge-covering for $V^{\prime}$ if there exists an edge $(x, y) \in E^{\prime}$ for any vertex $x \in V^{\prime}$. For $y \in V(G)$, let $\beta^{\prime}[y]$ be the minimum cardinality over all edge-coverings for $N_{G}^{-}[y]$.

Theorem 193 (Huang and $\mathrm{Xu}[36], 2008)$. If $G$ is a $k$-regular graph with order $\gamma(G)(k+1)$, then $b(G) \leqslant \beta^{\prime}[y]$ for any $y \in$ $V(G)$.

The upper bound on $b(G)$ given in Theorem 193 is tight in view of $b\left(C_{n}\right)$ for a cycle or a directed cycle $C_{n}$ (see Theorems 1 and 185 , resp.).

It is easy to see that for a $k$-regular graph $G,\lceil(k+1) / 2\rceil \leqslant$ $\beta^{\prime}[y] \leqslant k$ when $G$ is undirected and $\beta^{\prime}[y]=k+1$ when $G$ is directed. By this fact and Lemma 189, the following theorem is merely a simple combination of Theorems 191 and 193.

Theorem 194 (Huang and $\mathrm{Xu}[36], 2008$ ). Let $G$ be a vertextransitive graph of degree $k$. If $G$ has an efficient dominating set, then

$$
\begin{gathered}
\left\lceil\frac{k+1}{2}\right\rceil \leqslant b(G) \leqslant k \text { if } G \text { is undirected, } \\
b(G)=k+1 \text { if } G \text { is directed. }
\end{gathered}
$$

Theorem 195 (Huang and $\mathrm{Xu}$ [36], 2008). If $\mathrm{G}$ is an undirected vertex-transitive cubic graph with order $4 \gamma(G)$ and girth $g(G) \leqslant 5$, then $b(G)=2$.

The proof of Theorem 195 leads to a byproduct. If $g=$ 5 , let $\left(u_{1}, u_{2}, u_{3}, u_{4}, u_{5}\right)$ be a cycle, and let $\mathscr{D}_{i}$ be the family of efficient dominating sets containing $u_{i}$ for each $i \in$ $\{1,2,3,4,5\}$. Then $\mathscr{D}_{i} \cap \mathscr{D}_{j}=\emptyset$ for $i=1,2, \ldots, 5$. Then $G$ has at least $5 s$ efficient dominating sets, where $s=s(G)$. But there are only $4 s(=n s / \gamma)$ distinct efficient dominating sets in $G$. This contradiction implies that an undirected vertex-transitive cubic graph with girth five has no efficient dominating sets. But a similar argument for $g(G)=3,4$ or $g(G) \geqslant 6$ could not give any contradiction. This is consistent with the result that $\operatorname{CCC}(n)$, a vertex-transitive cubic graph with girth $n$ if $3 \leqslant n \leqslant 8$, or girth 8 if $n \geqslant 9$, has efficient dominating sets for all $n \geqslant 3$ except $n=5$ (see Theorem 199 in the following subsection).

11.2. Results for Cayley Graphs. In this subsection, we use Theorem 194 to determine the exact values or approximative values of bondage numbers for some special vertex-transitive graphs by characterizing the existence of efficient dominating sets in these graphs.

Let $\Gamma$ be a nontrivial finite group, $S$ a nonempty subset of $\Gamma$ without the identity element of $\Gamma$. A digraph $G$ is defined as follows:

$$
\begin{aligned}
V(G)=\Gamma, \quad(x, y) \in E(G) \Longleftrightarrow & x^{-1} y \in S \\
& \text { for any } x, y \in \Gamma
\end{aligned}
$$

is called a Cayley digraph of the group $\Gamma$ with respect to $S$, denoted by $C_{\Gamma}(S)$. If $S^{-1}=\left\{s^{-1}: s \in S\right\}=S$; then $C_{\Gamma}(S)$ is symmetric and is called a Cayley undirected graph, a Cayley graph for short. Cayley graphs or digraphs are certainly vertex-transitive (see Xu [1]).

A circulant graph $G(n ; S)$ of order $n$ is a Cayley graph $C_{Z_{n}}(S)$, where $Z_{n}=\{0, \ldots, n-1\}$ is the addition group of order $n$ and $S$ is a nonempty subset of $Z_{n}$ without the identity element and, hence, is a vertex-transitive digraph of degree $|S|$. If $S^{-1}=S$, then $G(n ; S)$ is an undirected graph. If $S=\{1, k\}$, where $2 \leqslant k \leqslant n-2$, we write $G(n ; 1, k)$ for $G(n ;\{1, k\})$ or $G(n ;\{ \pm 1, \pm k\})$ and call it a double-loop circulant graph.

Huang and $\mathrm{Xu}$ [36] showed that, for directed $G=$ $G(n ; 1, k),\lceil n / 3\rceil \leqslant \gamma(G) \leqslant\lceil n / 2\rceil$ and $G$ has an efficient dominating set if and only if $3 \mid n$ and $k \equiv 2(\bmod 3)$; for directed $G=G(n ; 1, k)$ and $k \neq n / 2,\lceil n / 5\rceil \leqslant \gamma(G) \leqslant\lceil n / 3\rceil$ and $G$ has an efficient dominating set if and only if $5 \mid n$ and $k \equiv \pm 2(\bmod 5)$. By Theorem 194 , we obtain the bondage number of a double loop circulant graph if it has an efficient dominating set.

Theorem 196 (Huang and $\mathrm{Xu}[36], 2008)$. Let $\mathrm{G}$ be a doubleloop circulant graph $G(n ; 1, k)$. If $G$ is directed with 3 | $n$ and $k \equiv 2(\bmod 3)$, or $G$ is undirected with $5 \mid n$ and $k \equiv \pm 2(\bmod 5)$, then $b(G)=3$.

The $m \times n$ torus is the Cartesian product $C_{m} \times C_{n}$ of two cycles and is a Cayley graph $C_{Z_{m} \times Z_{n}}(S)$, where $S=$ $\{(0,1),(1,0)\}$ for directed cycles and $S=\{(0, \pm 1),( \pm 1,0)\}$ for undirected cycles and, hence, is vertex-transitive. $\mathrm{Gu}$ et al. [144] showed that the undirected torus $C_{m} \times C_{n}$ has an efficient dominating set if and only if both $m$ and $n$ are multiples of 5. Huang and $\mathrm{Xu}$ [36] showed that the directed torus $C_{m} \times C_{n}$ has an efficient dominating set if and only if both $m$ and $n$ are multiples of 3 . Moreover, they found a necessary condition for a dominating set containing the vertex $(0,0)$ in $C_{m} \times C_{n}$ to be efficient and obtained the following result. 
Theorem 197 (Huang and $\mathrm{Xu}[36], 2008$ ). Let $G=C_{m} \times C_{n}$. If $G$ is undirected and both $m$ and $n$ are multiples of 5 or if $G$ is directed and both $m$ and $n$ are multiples of 3 , then $b(G)=3$.

The hypercube $Q_{n}$ is the Cayley graph $C_{\Gamma}(S)$, where $\Gamma=Z_{2} \times \cdots \times Z_{2}=\left(Z_{2}\right)^{n}$ and $S=$ $\{100 \cdots 0,010 \cdots 0, \ldots, 00 \cdots 01\}$. Lee [143] showed that $Q_{n}$ has an efficient dominating set if and only if $n=2^{m}-1$ for a positive integer $m$. By Theorem 194, the following result is obtained immediately.

Theorem 198 (Huang and $\mathrm{Xu}$ [36], 2008). If $n=2^{m}-1$ for $a$ positive integer $m$, then $2^{m-1} \leqslant b\left(Q_{n}\right) \leqslant 2^{m}-1$.

Problem 10. Determine the exact value of $b\left(Q_{n}\right)$ for $n=2^{m}-1$.

The $n$-dimensional cube-connected cycle, denoted by $\operatorname{CCC}(n)$, is constructed from the $n$-dimensional hypercube $Q_{n}$ by replacing each vertex $x$ in $Q_{n}$ with an undirected cycle $C_{n}$ of length $n$ and linking the $i$ th vertex of the $C_{n}$ to the $i$ th neighbor of $x$. It has been proved that $\operatorname{CCC}(n)$ is a Cayley graph and, hence, is a vertex-transitive graph with degree 3. Van Wieren et al. [147] proved that CCC $(n)$ has an efficient dominating set if and only if $n \neq 5$. From Theorems 194 and 195, the following result can be derived.

Theorem 199 (Huang and Xu [36], 2008). Let $G=\operatorname{CCC}(n)$ be the $n$-dimensional cube-connected cycles with $n \geqslant 3$ and $n \neq 5$. Then $\gamma(G)=n 2^{n-2}$ and $2 \leqslant b(G) \leqslant 3$. In addition, $b(C C C(3))=b(C C C(4))=2$.

Problem 11. Determine the exact value of $b(\mathrm{CCC}(n))$ for $n \geqslant$ 5 .

\section{Acknowledgments}

This work was supported by NNSF of China (Grants nos. $11071233,61272008)$. The author would like to express his gratitude to the anonymous referees for their kind suggestions and comments on the original paper, to Professor Sheikholeslami and Professor Jafari Rad for sending the references [128] and [81], which resulted in this version.

\section{References}

[1] J.-M. Xu, Theory and Application of Graphs, vol. 10 of Network Theory and Applications, Kluwer Academic, Dordrecht, The Netherlands, 2003.

[2] S. T. Hedetniemi and R. C. Laskar, "Bibliography on domination in graphs and some basic definitions of domination parameters," Discrete Mathematics, vol. 86, no. 1-3, pp. 257-277, 1990.

[3] T. W. Haynes, S. T. Hedetniemi, and P. J. Slater, Fundamentals of Domination in Graphs, vol. 208 of Monographs and Textbooks in Pure and Applied Mathematics, Marcel Dekker, New York, NY, USA, 1998.

[4] T. W. Haynes, S. T. Hedetniemi, and P. J. Slater, Eds., Domination in Graphs, vol. 209 of Monographs and Textbooks in Pure and Applied Mathematics, Marcel Dekker, New York, NY, USA, 1998.

[5] M. R. Garey and D. S. Johnson, Computers and Intractability, W. H. Freeman and Company, San Francisco, Calif, USA, 1979.
[6] H. B. Walikar and B. D. Acharya, "Domination critical graphs," National Academy Science Letters, vol. 2, pp. 70-72, 1979.

[7] D. Bauer, F. Harary, J. Nieminen, and C. L. Suffel, "Domination alteration sets in graphs," Discrete Mathematics, vol. 47, no. 2-3, pp. 153-161, 1983.

[8] J. F. Fink, M. S. Jacobson, L. F. Kinch, and J. Roberts, "The bondage number of a graph," Discrete Mathematics, vol. 86, no. $1-3$, pp. 47-57, 1990.

[9] F.-T. Hu and J.-M. Xu, "The bondage number of $(n-3)$-regular graph $G$ of order $n$," Ars Combinatoria. to appear.

[10] U. Teschner, "New results about the bondage number of a graph," Discrete Mathematics, vol. 171, no. 1-3, pp. 249-259, 1997.

[11] B. L. Hartnell and D. F. Rall, "A bound on the size of a graph with given order and bondage number," Discrete Mathematics, vol. 197-198, pp. 409-413, 1999, 16th British Combinatorial Conference (London, 1997).

[12] B. L. Hartnell and D. F. Rall, "A characterization of trees in which no edge is essential to the domination number," Ars Combinatoria, vol. 33, pp. 65-76, 1992.

[13] J. Topp and P. D. Vestergaard, " $\alpha_{k}$ - and $\gamma_{k}$-stable graphs," Discrete Mathematics, vol. 212, no. 1-2, pp. 149-160, 2000.

[14] A. Meir and J. W. Moon, "Relations between packing and covering numbers of a tree," Pacific Journal of Mathematics, vol. 61, no. 1, pp. 225-233, 1975.

[15] B. L. Hartnell, L. K. Jørgensen, P. D. Vestergaard, and C. A. Whitehead, "Edge stability of the $k$-domination number of trees," Bulletin of the Institute of Combinatorics and Its Applications, vol. 22, pp. 31-40, 1998.

[16] F.-T. Hu and J.-M. Xu, "On the complexity of the bondage and reinforcement problems," Journal of Complexity, vol. 28, no. 2, pp. 192-201, 2012.

[17] B. L. Hartnell and D. F. Rall, "Bounds on the bondage number of a graph," Discrete Mathematics, vol. 128, no. 1-3, pp. 173-177, 1994.

[18] Y.-L. Wang, “On the bondage number of a graph," Discrete Mathematics, vol. 159, no. 1-3, pp. 291-294, 1996.

[19] G. H. Fricke, T. W. Haynes, S. M. Hedetniemi, S. T. Hedetniemi, and R. C. Laskar, "Excellent trees," Bulletin of the Institute of Combinatorics and Its Applications, vol. 34, pp. 27-38, 2002.

[20] V. Samodivkin, "The bondage number of graphs: good and bad vertices," Discussiones Mathematicae. Graph Theory, vol. 28, no. 3, pp. 453-462, 2008.

[21] J. E. Dunbar, T. W. Haynes, U. Teschner, and L. Volkmann, "Bondage, insensitivity, and reinforcement," in Domination in Graphs, vol. 209 of Monographs and Textbooks in Pure and Applied Mathematics, pp. 471-489, Dekker, New York, NY, USA, 1998.

[22] H. Liu and L. Sun, "The bondage and connectivity of a graph," Discrete Mathematics, vol. 263, no. 1-3, pp. 289-293, 2003.

[23] A.-H. Esfahanian and S. L. Hakimi, "On computing a conditional edge-connectivity of a graph," Information Processing Letters, vol. 27, no. 4, pp. 195-199, 1988.

[24] R. C. Brigham, P. Z. Chinn, and R. D. Dutton, "Vertex domination-critical graphs," Networks, vol. 18, no. 3, pp. 173-179, 1988.

[25] V. Samodivkin, "Domination with respect to nondegenerate properties: bondage number," The Australasian Journal of Combinatorics, vol. 45, pp. 217-226, 2009.

[26] U. Teschner, "A new upper bound for the bondage number of graphs with small domination number," The Australasian Journal of Combinatorics, vol. 12, pp. 27-35, 1995. 
[27] J. Fulman, D. Hanson, and G. MacGillivray, "Vertex domination-critical graphs," Networks, vol. 25, no. 2, pp. 41-43, 1995.

[28] U. Teschner, "A counterexample to a conjecture on the bondage number of a graph," Discrete Mathematics, vol. 122, no. 1-3, pp. 393-395, 1993.

[29] U. Teschner, "The bondage number of a graph $G$ can be much greater than $\Delta(G)$," Ars Combinatoria, vol. 43, pp. 81-87, 1996.

[30] L. Volkmann, "On graphs with equal domination and covering numbers," Discrete Applied Mathematics, vol. 51, no. 1-2, pp. 211217, 1994

[31] L. A. Sanchis, "Maximum number of edges in connected graphs with a given domination number," Discrete Mathematics, vol. 87, no. 1, pp. 65-72, 1991.

[32] S. Klavžar and N. Seifter, "Dominating Cartesian products of cycles," Discrete Applied Mathematics, vol. 59, no. 2, pp. 129-136, 1995.

[33] H. K. Kim, "A note on Vizing's conjecture about the bondage number," Korean Journal of Mathematics, vol. 10, no. 2, pp. 3942, 2003.

[34] M. Y. Sohn, X. Yuan, and H. S. Jeong, "The bondage number of $C_{3} \times C_{n}$, Journal of the Korean Mathematical Society, vol. 44, no. 6, pp. 1213-1231, 2007.

[35] L. Kang, M. Y. Sohn, and H. K. Kim, "Bondage number of the discrete torus $C_{n} \times C_{4}$," Discrete Mathematics, vol. 303, no. 1-3, pp. 80-86, 2005.

[36] J. Huang and J.-M. Xu, "The bondage numbers and efficient dominations of vertex-transitive graphs," Discrete Mathematics, vol. 308, no. 4, pp. 571-582, 2008.

[37] C. J. Xiang, X. Yuan, and M. Y. Sohn, "Domination and bondage number of $C_{3} \times C_{n}$," Ars Combinatoria, vol. 97, pp. 299-310, 2010.

[38] M. S. Jacobson and L. F. Kinch, "On the domination number of products of graphs. I," Ars Combinatoria, vol. 18, pp. 33-44, 1984.

[39] Y.-Q. Li, "A note on the bondage number of a graph," Chinese Quarterly Journal of Mathematics, vol. 9, no. 4, pp. 1-3, 1994.

[40] F.-T. Hu and J.-M. Xu, "Bondage number of mesh networks," Frontiers of Mathematics in China, vol. 7, no. 5, pp. 813-826, 2012.

[41] R. Frucht and F. Harary, "On the corona of two graphs," Aequationes Mathematicae, vol. 4, pp. 322-325, 1970.

[42] K. Carlson and M. Develin, "On the bondage number of planar and directed graphs," Discrete Mathematics, vol.306, no. 8-9, pp. 820-826, 2006.

[43] U. Teschner, "On the bondage number of block graphs," Ars Combinatoria, vol. 46, pp. 25-32, 1997.

[44] J. Huang and J.-M. Xu, "Note on conjectures of bondage numbers of planar graphs," Applied Mathematical Sciences, vol. 6, no. 65-68, pp. 3277-3287, 2012.

[45] L. Kang and J. Yuan, "Bondage number of planar graphs," Discrete Mathematics, vol. 222, no. 1-3, pp. 191-198, 2000.

[46] M. Fischermann, D. Rautenbach, and L. Volkmann, "Remarks on the bondage number of planar graphs," Discrete Mathematics, vol. 260, no. 1-3, pp. 57-67, 2003.

[47] J. Hou, G. Liu, and J. Wu, "Bondage number of planar graphs without small cycles," Utilitas Mathematica, vol. 84, pp. 189-199, 2011.

[48] J. Huang and J.-M. Xu, "The bondage numbers of graphs with small crossing numbers," Discrete Mathematics, vol. 307, no. 15, pp. 1881-1897, 2007.

[49] Q. Ma, S. Zhang, and J. Wang, "Bondage number of 1-planar graph,” Applied Mathematics, vol. 1, no. 2, pp. 101-103, 2010.
[50] J. Hou and G. Liu, "A bound on the bondage number of toroidal graphs," Discrete Mathematics, Algorithms and Applications, vol. 4, no. 3, Article ID 1250046, 5 pages, 2012.

[51] Y.-C. Cao, J.-M. Xu, and X.-R. Xu, "On bondage number of toroidal graphs," Journal of University of Science and Technology of China, vol. 39, no. 3, pp. 225-228, 2009.

[52] Y.-C. Cao, J. Huang, and J.-M. Xu, "The bondage number of graphs with crossing number less than four," Ars Combinatoria. to appear.

[53] H. K. Kim, "On the bondage numbers of some graphs," Korean Journal of Mathematics, vol. 11, no. 2, pp. 11-15, 2004.

[54] A. Gagarin and V. Zverovich, "Upper bounds for the bondage number of graphs on topological surfaces," Discrete Mathematics, 2012.

[55] J. Huang, "An improved upper bound for the bondage number of graphs on surfaces," Discrete Mathematics, vol. 312, no. 18, pp. 2776-2781, 2012.

[56] A. Gagarin and V. Zverovich, "The bondage number of graphs on topological surfaces and Teschner's conjecture," Discrete Mathematics, vol. 313, no. 6, pp. 796-808, 2013.

[57] V. Samodivkin, "Note on the bondage number of graphs on topological surfaces," http://arxiv.org/abs/1208.6203 .

[58] E. J. Cockayne, R. M. Dawes, and S. T. Hedetniemi, “Total domination in graphs," Networks, vol. 10, no. 3, pp. 211-219, 1980.

[59] R. Laskar, J. Pfaff, S. M. Hedetniemi, and S. T. Hedetniemi, "On the algorithmic complexity of total domination," Society for Industrial and Applied Mathematics, vol. 5, no. 3, pp. 420-425, 1984.

[60] J. Pfaff, R. C. Laskar, and S. T. Hedetniemi, "NP-completeness of total and connected domination and irredundance for bipartite graphs," Tech. Rep. 428, Department of Mathematical Sciences, Clemson University, 1983.

[61] M. A. Henning, "A survey of selected recent results on total domination in graphs," Discrete Mathematics, vol. 309, no. 1, pp. 32-63, 2009.

[62] V. R. Kulli and D. K. Patwari, "The total bondage number of a graph," in Advances in Graph Theory, pp. 227-235, Vishwa, Gulbarga, India, 1991.

[63] F.-T. Hu, Y. Lu, and J.-M. Xu, "The total bondage number of grid graphs," Discrete Applied Mathematics, vol. 160, no. 16-17, pp. 2408-2418, 2012.

[64] J. Cao, M. Shi, and B. Wu, "Research on the total bondage number of a spectial network," in Proceedings of the $3 \mathrm{rd}$ International Joint Conference on Computational Sciences and Optimization (CSO '10), pp. 456-458, May 2010.

[65] N. Sridharan, MD. Elias, and V. S. A. Subramanian, "Total bondage number of a graph," AKCE International Journal of Graphs and Combinatorics, vol. 4, no. 2, pp. 203-209, 2007.

[66] N. Jafari Rad and J. Raczek, "Some progress on total bondage in graphs," Graphs and Combinatorics, 2011.

[67] T. W. Haynes and P. J. Slater, "Paired-domination and the paired-domatic number," Congressus Numerantium, vol. 109, pp. 65-72, 1995.

[68] T. W. Haynes and P. J. Slater, "Paired-domination in graphs," Networks, vol. 32, no. 3, pp. 199-206, 1998.

[69] X. Hou, "A characterization of $2 \gamma, \gamma_{P}$-trees," Discrete Mathematics, vol. 308, no. 15, pp. 3420-3426, 2008.

[70] K. E. Proffitt, T. W. Haynes, and P. J. Slater, "Paired-domination in grid graphs," Congressus Numerantium, vol. 150, pp. 161-172, 2001. 
[71] H. Qiao, L. Kang, M. Cardei, and D.-Z. Du, "Paired-domination of trees," Journal of Global Optimization, vol. 25, no. 1, pp. 43-54, 2003.

[72] E. Shan, L. Kang, and M. A. Henning, "A characterization of trees with equal total domination and paired-domination numbers," The Australasian Journal of Combinatorics, vol. 30, pp. 31-39, 2004.

[73] J. Raczek, "Paired bondage in trees," Discrete Mathematics, vol. 308, no. 23, pp. 5570-5575, 2008.

[74] J. A. Telle and A. Proskurowski, "Algorithms for vertex partitioning problems on partial $k$-trees," SIAM Journal on Discrete Mathematics, vol. 10, no. 4, pp. 529-550, 1997.

[75] G. S. Domke, J. H. Hattingh, M. A. Henning, and L. R. Markus, "Restrained domination in trees," Discrete Mathematics, vol. 211, no. 1-3, pp. 1-9, 2000.

[76] G. S. Domke, J. H. Hattingh, M. A. Henning, and L. R. Markus, "Restrained domination in graphs with minimum degree two," Journal of Combinatorial Mathematics and Combinatorial Computing, vol. 35, pp. 239-254, 2000.

[77] G. S. Domke, J. H. Hattingh, S. T. Hedetniemi, R. C. Laskar, and L. R. Markus, "Restrained domination in graphs," Discrete Mathematics, vol. 203, no. 1-3, pp. 61-69, 1999.

[78] J. H. Hattingh and E. J. Joubert, "An upper bound for the restrained domination number of a graph with minimum degree at least two in terms of order and minimum degree," Discrete Applied Mathematics, vol. 157, no. 13, pp. 2846-2858, 2009.

[79] M. A. Henning, "Graphs with large restrained domination number," Discrete Mathematics, vol. 197-198, pp. 415-429, 1999, 16th British Combinatorial Conference (London, 1997).

[80] J. H. Hattingh and A. R. Plummer, "Restrained bondage in graphs," Discrete Mathematics, vol. 308, no. 23, pp. 5446-5453, 2008.

[81] N. Jafari Rad, R. Hasni, J. Raczek, and L. Volkmann, "Total restrained bondage in graphs," Acta Mathematica Sinica, vol. 29, no. 6, pp. 1033-1042, 2013.

[82] J. Cyman and J. Raczek, "On the total restrained domination number of a graph," The Australasian Journal of Combinatorics, vol. 36, pp. 91-100, 2006.

[83] M. A. Henning, "Graphs with large total domination number," Journal of Graph Theory, vol. 35, no. 1, pp. 21-45, 2000.

[84] D.-X. Ma, X.-G. Chen, and L. Sun, "On total restrained domination in graphs," Czechoslovak Mathematical Journal, vol. 55, no. 1, pp. 165-173, 2005.

[85] B. Zelinka, "Remarks on restrained domination and total restrained domination in graphs," Czechoslovak Mathematical Journal, vol. 55, no. 2, pp. 393-396, 2005.

[86] W. Goddard and M. A. Henning, "Independent domination in graphs: a survey and recent results," Discrete Mathematics, vol. 313, no. 7, pp. 839-854, 2013.

[87] J. H. Zhang, H. L. Liu, and L. Sun, "Independence bondage number and reinforcement number of some graphs," Transactions of Beijing Institute of Technology, vol. 23, no. 2, pp. 140-142, 2003.

[88] K. D. Dharmalingam, Studies in Graph Theory-equitable domination and bottleneck domination [Ph.D. thesis], Madurai Kamaraj University, Madurai, India, 2006.

[89] G. Deepak, N. D. Soner, and A. Alwardi, "The equitable bondage number of a graph," Research Journal of Pure Algebra, vol. 1, no. 9, pp. 209-212, 2011.
[90] E. Sampathkumar and H. B. Walikar, "The connected domination number of a graph," Journal of Mathematical and Physical Sciences, vol. 13, no. 6, pp. 607-613, 1979.

[91] K. White, M. Farber, and W. Pulleyblank, "Steiner trees, connected domination and strongly chordal graphs," Networks, vol. 15, no. 1, pp. 109-124, 1985.

[92] M. Cadei, M. X. Cheng, X. Cheng, and D.-Z. Du, "Connected domination in Ad Hoc wireless networks," in Proceedings of the 6th International Conference on Computer Science and Informatics (CS\&I '02), 2002.

[93] J. F. Fink and M. S. Jacobson, "n-domination in graphs," in Graph Theory with Applications to Algorithms and Computer Science, Wiley-Interscience Publications, pp. 283-300, Wiley, New York, NY, USA, 1985.

[94] M. Chellali, O. Favaron, A. Hansberg, and L. Volkmann, "kdomination and k-independence in graphs: a survey," Graphs and Combinatorics, vol. 28, no. 1, pp. 1-55, 2012.

[95] Y. Lu, X. Hou, J.-M. Xu, and N. Li, “Trees with unique minimum p-dominating sets," Utilitas Mathematica, vol. 86, pp. 193-205, 2011.

[96] Y. Lu and J.-M. Xu, “The p-bondage number of trees," Graphs and Combinatorics, vol. 27, no. 1, pp. 129-141, 2011.

[97] Y. Lu and J.-M. Xu, “The 2-domination and 2-bondage numbers of grid graphs. A manuscript," http://arxiv.org/abs/1204.4514 .

[98] M. Krzywkowski, "Non-isolating 2-bondage in graphs," The Journal of the Mathematical Society of Japan, vol. 64, no. 4, 2012.

[99] M. A. Henning, O. R. Oellermann, and H. C. Swart, "Bounds on distance domination parameters," Journal of Combinatorics, Information \& System Sciences, vol. 16, no. 1, pp. 11-18, 1991.

[100] F. Tian and J.-M. Xu, "Bounds for distance domination numbers of graphs," Journal of University of Science and Technology of China, vol. 34, no. 5, pp. 529-534, 2004.

[101] F. Tian and J.-M. Xu, "Distance domination-critical graphs," Applied Mathematics Letters, vol. 21, no. 4, pp. 416-420, 2008.

[102] F. Tian and J.-M. Xu, "A note on distance domination numbers of graphs," The Australasian Journal of Combinatorics, vol. 43, pp. 181-190, 2009.

[103] F. Tian and J.-M. Xu, "Average distances and distance domination numbers," Discrete Applied Mathematics, vol. 157, no. 5, pp. 1113-1127, 2009.

[104] Z.-L. Liu, F. Tian, and J.-M. Xu, "Probabilistic analysis of upper bounds for 2-connected distance $k$-dominating sets in graphs," Theoretical Computer Science, vol. 410, no. 38-40, pp. 3804$3813,2009$.

[105] M. A. Henning, O. R. Oellermann, and H. C. Swart, "Distance domination critical graphs," Journal of Combinatorial Mathematics and Combinatorial Computing, vol. 44, pp. 33-45, 2003.

[106] T. J. Bean, M. A. Henning, and H. C. Swart, "On the integrity of distance domination in graphs," The Australasian Journal of Combinatorics, vol. 10, pp. 29-43, 1994.

[107] M. Fischermann and L. Volkmann, "A remark on a conjecture for the ( $k, p)$-domination number," Utilitas Mathematica, vol. 67, pp. 223-227, 2005.

[108] T. Korneffel, D. Meierling, and L. Volkmann, "A remark on the (2,2)-domination number," Discussiones Mathematicae. Graph Theory, vol. 28, no. 2, pp. 361-366, 2008.

[109] Y. Lu, X. Hou, and J.-M. Xu, "On the (2,2)-domination number of trees," Discussiones Mathematicae. Graph Theory, vol. 30, no. 2, pp. 185-199, 2010. 
[110] H. Li and J.-M. Xu, “( $d, m)$-domination numbers of $\mathrm{m}$ connected graphs,” Rapports de Recherche 1130, LRI, Université Paris-Sud, 1997.

[111] S. M. Hedetniemi, S. T. Hedetniemi, and T. V. Wimer, "Linear time resource allocation algorithms for trees," Tech. Rep. URI014, Department of Mathematical Sciences, Clemson University, 1987.

[112] M. Farber, "Domination, independent domination, and duality in strongly chordal graphs," Discrete Applied Mathematics, vol. 7, no. 2, pp. 115-130, 1984.

[113] G. S. Domke and R. C. Laskar, "The bondage and reinforcement numbers of $\gamma_{f}$ for some graphs," Discrete Mathematics, vol. 167168, pp. 249-259, 1997.

[114] G. S. Domke, S. T. Hedetniemi, and R. C. Laskar, "Fractional packings, coverings, and irredundance in graphs," vol. 66, pp. 227-238, 1988.

[115] E. J. Cockayne, P. A. Dreyer, Jr., S. M. Hedetniemi, and S. T. Hedetniemi, "Roman domination in graphs," Discrete Mathematics, vol. 278, no. 1-3, pp. 11-22, 2004.

[116] I. Stewart, "Defend the roman empire," Scientific American, vol. 281, pp. 136-139, 1999.

[117] C. S. ReVelle and K. E. Rosing, "Defendens imperium romanum: a classical problem in military strategy," The American Mathematical Monthly, vol. 107, no. 7, pp. 585-594, 2000.

[118] A. Bahremandpour, F.-T. Hu, S. M. Sheikholeslami, and J.-M. $\mathrm{Xu}$, "On the Roman bondage number of a graph," Discrete Mathematics, Algorithms and Applications, vol. 5, no. 1, Article ID 1350001, 15 pages, 2013.

[119] N. Jafari Rad and L. Volkmann, "Roman bondage in graphs," Discussiones Mathematicae. Graph Theory, vol. 31, no. 4, pp. 763-773, 2011.

[120] K. Ebadi and L. PushpaLatha, "Roman bondage and Roman reinforcement numbers of a graph," International Journal of Contemporary Mathematical Sciences, vol. 5, pp. 1487-1497, 2010.

[121] N. Dehgardi, S. M. Sheikholeslami, and L. Volkman, "On the Roman $k$-bondage number of a graph," AKCE International Journal of Graphs and Combinatorics, vol. 8, pp. 169-180, 2011.

[122] S. Akbari and S. Qajar, "A note on Roman bondage number of graphs," Ars Combinatoria. to Appear.

[123] F.-T. Hu and J.-M. Xu, "Roman bondage numbers of some graphs," http://arxiv.org/abs/1109.3933 .

[124] N. Jafari Rad and L. Volkmann, "On the Roman bondage number of planar graphs," Graphs and Combinatorics, vol. 27, no. 4, pp. 531-538, 2011.

[125] S. Akbari, M. Khatirinejad, and S. Qajar, "A note on the roman bondage number of planar graphs," Graphs and Combinatorics, 2012.

[126] B. Brešar, M. A. Henning, and D. F. Rall, "Rainbow domination in graphs," Taiwanese Journal of Mathematics, vol. 12, no. 1, pp. 213-225, 2008.

[127] B. L. Hartnell and D. F. Rall, "On dominating the Cartesian product of a graph and $\alpha \mathrm{k}$," Discussiones Mathematicae. Graph Theory, vol. 24, no. 3, pp. 389-402, 2004.

[128] N. Dehgardi, S. M. Sheikholeslami, and L. Volkman, "The $k$ rainbow bondage number of a graph," to appear in Discrete Applied Mathematics.

[129] J. von Neumann and O. Morgenstern, Theory of Games and Economic Behavior, Princeton University Press, Princeton, NJ, USA, 1944.
[130] C. Berge, Theoríe des Graphes et ses Applications, 1958.

[131] O. Ore, Theory of Graphs, vol. 38, American Mathematical Society Colloquium Publications, 1962.

[132] E. Shan and L. Kang, "Bondage number in oriented graphs," Ars Combinatoria, vol. 84, pp. 319-331, 2007.

[133] J. Huang and J.-M. Xu, "The bondage numbers of extended de Bruijn and Kautz digraphs," Computers \& Mathematics with Applications, vol. 51, no. 6-7, pp. 1137-1147, 2006.

[134] J. Huang and J.-M. Xu, "The total domination and total bondage numbers of extended de Bruijn and Kautz digraphs," Computers \& Mathematics with Applications, vol. 53, no. 8, pp. 1206-1213, 2007.

[135] Y. Shibata and Y. Gonda, "Extension of de Bruijn graph and Kautz graph," Computers \& Mathematics with Applications, vol. 30, no. 9, pp. 51-61, 1995.

[136] X. Zhang, J. Liu, and J. Meng, “The bondage number in complete t-partite digraphs," Information Processing Letters, vol. 109, no. 17, pp. 997-1000, 2009.

[137] D. W. Bange, A. E. Barkauskas, and P. J. Slater, "Efficient dominating sets in graphs," in Applications of Discrete Mathematics, pp. 189-199, SIAM, Philadelphia, Pa, USA, 1988.

[138] M. Livingston and Q. F. Stout, "Perfect dominating sets," Congressus Numerantium, vol. 79, pp. 187-203, 1990.

[139] L. Clark, "Perfect domination in random graphs," Journal of Combinatorial Mathematics and Combinatorial Computing, vol. 14, pp. 173-182, 1993.

[140] D. W. Bange, A. E. Barkauskas, L. H. Host, and L. H. Clark, "Efficient domination of the orientations of a graph," Discrete Mathematics, vol. 178, no. 1-3, pp. 1-14, 1998.

[141] A. E. Barkauskas and L. H. Host, "Finding efficient dominating sets in oriented graphs," Congressus Numerantium, vol. 98, pp. 27-32, 1993.

[142] I. J. Dejter and O. Serra, "Efficient dominating sets in Cayley graphs," Discrete Applied Mathematics, vol. 129, no. 2-3, pp. 319328, 2003.

[143] J. Lee, "Independent perfect domination sets in Cayley graphs," Journal of Graph Theory, vol. 37, no. 4, pp. 213-219, 2001.

[144] W. Gu, X. Jia, and J. Shen, "Independent perfect domination sets in meshes, tori and trees," Unpublished.

[145] N. Obradović, J. Peters, and G. Ružić, "Efficient domination in circulant graphs with two chord lengths," Information Processing Letters, vol. 102, no. 6, pp. 253-258, 2007.

[146] K. Reji Kumar and G. MacGillivray, "Efficient domination in circulant graphs," Discrete Mathematics, vol. 313, no. 6, pp. 767771, 2013.

[147] D. Van Wieren, M. Livingston, and Q. F. Stout, "Perfect dominating sets on cube-connected cycles," Congressus Numerantium, vol. 97, pp. 51-70, 1993. 


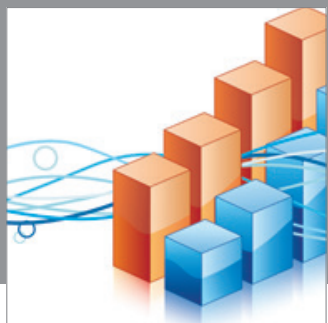

Advances in

Operations Research

mansans

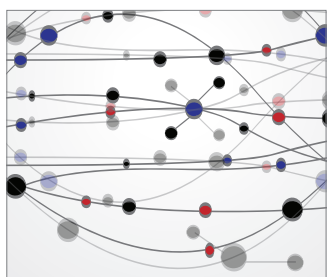

The Scientific World Journal
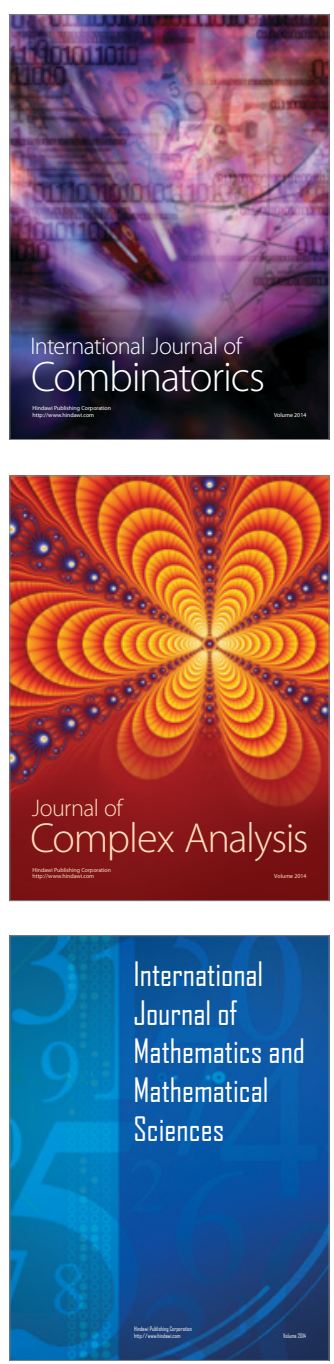
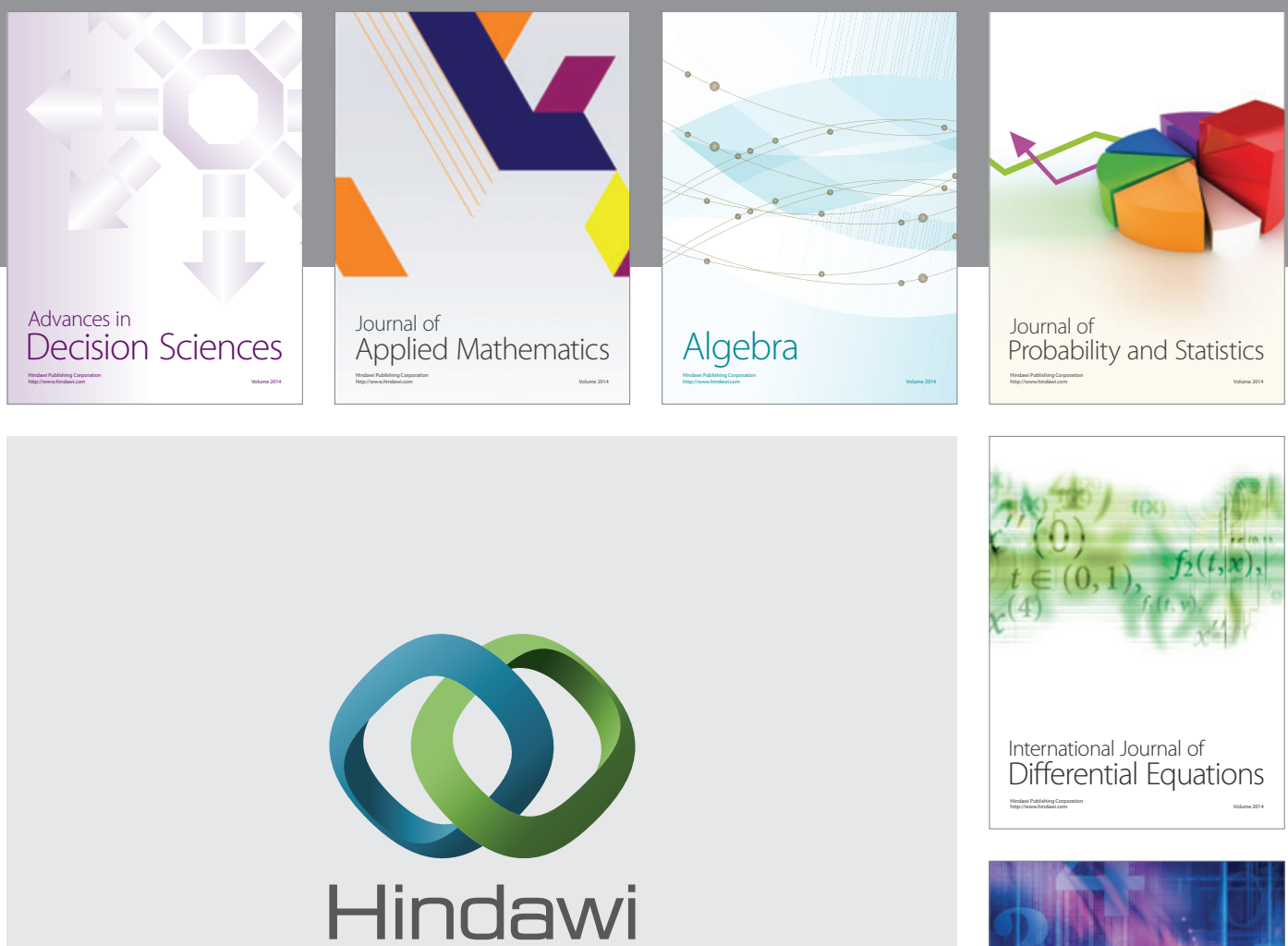

Submit your manuscripts at http://www.hindawi.com
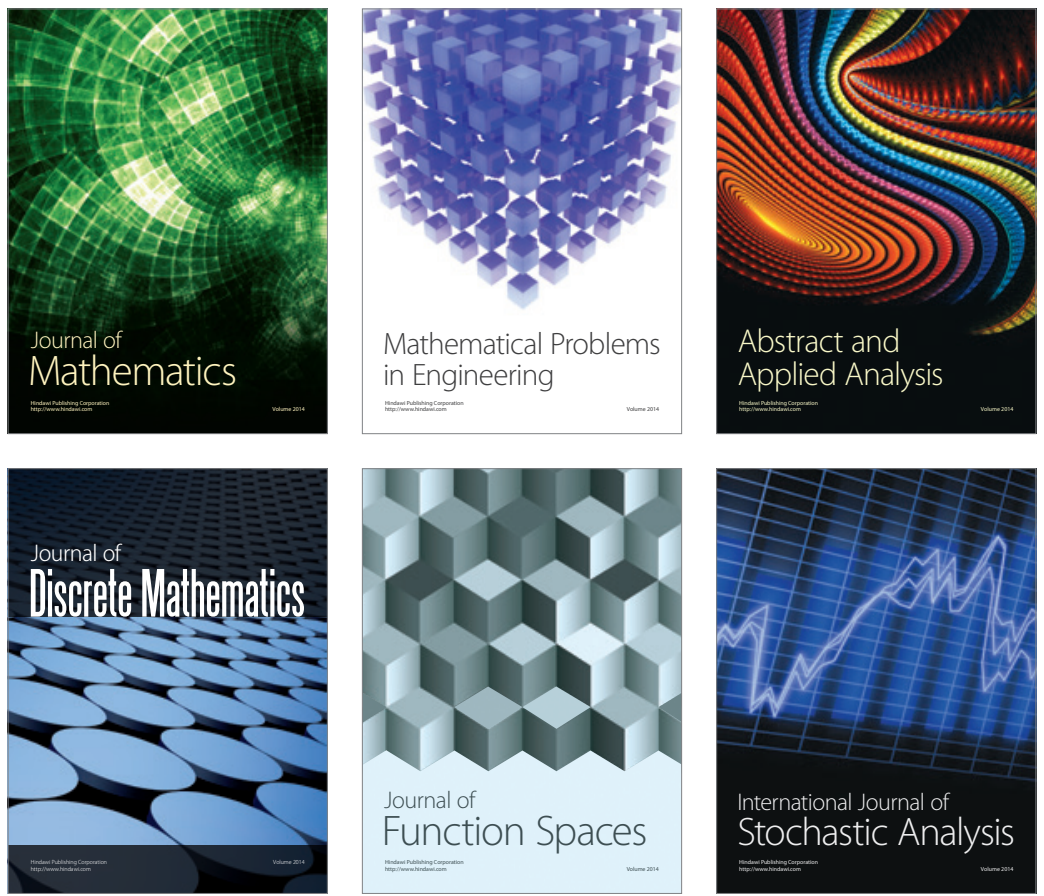

Journal of

Function Spaces

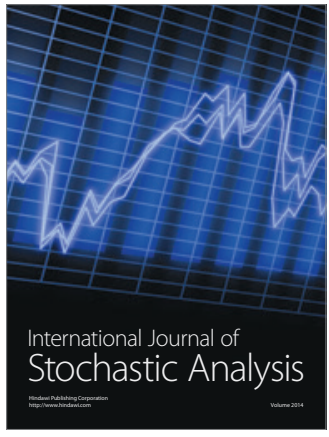

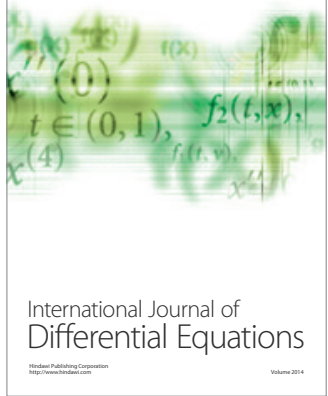
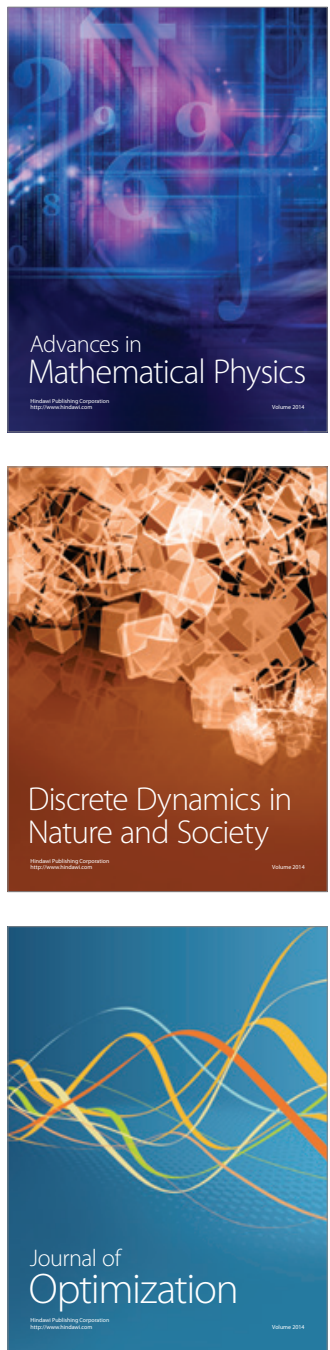